\title{
COMBINATORIAL DICHOTOMIES IN SET THEORY
}

\author{
STEVO TODORCEVIC
}

In this article we give an overview of a line of research in set theory that has reached a level of maturity and which, in our opinion, merits its being exposed to a more general audience. This line of research is concentrated on finding to which extent compactness fails at the level of first uncountable cardinal and to which extent it could be recovered at the level of some other not so large cardinal, the most interesting being of course the level of the second uncountable cardinal. While this is of great interest to set theorists, one of the main forces behind this line of research stems from its applicability to other areas of mathematics where one encounters structures that are not necessarily countable, and therefore one is likely to encounter problems of this kind. We have split this overview into three parts each presenting a set theoretic combinatorial principle imposing a degree of compactness at some small cardinal. The three principles are natural dichotomies about chromatic numbers for graphs and about ideals of countable subsets of some index set. They are all relatively easy to state and apply and are therefore accessible to mathematicians working in areas outside of set theory. Each of these three dichotomies has its axiomatic as well as its mathematical side and we went into some effort to show the close relationship between these. For example, we have tried to show that an abstract analysis of one of these three set theoretic principles can sometimes lead us to results that do not require additional axioms at all but which could have been otherwise difficult to discover directly. We have also tried to select examples with as broad range as possible but their choices could still reflect a personal taste. We have therefore included an extensive reference list where the reader can find a more complete view on this area of current research in set theory. Finally we mention that while this article is meant for a larger audience which is typically interested in a general overview, we have tried to make the article also interesting to experts working in this area by including some of the technicalities especially if they appear to us as important tools in this area. For the same reason we will be pointing out a number of possible directions for further research.

Our terminology and notation follows that of standard texts of set theory (see, for example, [53] and [67]). Recall that the Singular Cardinals Hypothesis, $\mathrm{SCH}$, is the statement

$$
(\forall \theta) \theta^{\operatorname{cf} \theta}=\theta^{+} \cdot 2^{\operatorname{cf} \theta} .
$$

It is a strong structural assumption about the universe of sets as it answers all questions about the arithmetic of infinite cardinals. It is for this reason one of the most studied such hypotheses of set theory especially in the context of covering properties of inner models of set theory. Another set of principles which is even more relevant from this point of view are the square principles such as $\square_{\kappa}$ and $\square(\kappa)$. Before we introduce these principles, recall that $C_{\alpha}(\alpha<\theta)$ is a $C$-sequence if $C_{\alpha}$ is a closed and unbounded subset of $\alpha$ for all $\alpha<\theta$. These combinatorial

Date: Bern, July 3, 4, and 5, 2008. 
principles $\square_{\kappa}$ and $\square(\kappa)$ assert the existence of $C$-sequences $C_{\alpha}(\alpha<\theta)$ for $\theta=\kappa^{+}$ and $\theta=\kappa$, respectively, with some extra properties most important of which is the coherence property stating that

$$
C_{\alpha}=C_{\beta} \cap \alpha \text { whenever } \alpha \text { is a limit point of } C_{\beta} .
$$

For $C_{\alpha}\left(\alpha<\kappa^{+}\right)$to form a $\square_{\kappa}$-sequence the sets $C_{\alpha}$ have to be moreover all of order type $\leq \kappa$. On the other hand, for $C_{\alpha}(\alpha<\theta)$ to form a $\square(\theta)$-sequence it has to be moreover nontrivial in the sense that there is no closed and unbounded subset $C$ of $\theta$ such that $C_{\alpha}=C \cap \alpha$ for every limit point $\alpha$ of $C$. Note that $\square\left(\omega_{1}\right)$ and in fact $\square_{\omega}$ hold $\$^{1}$ but the existence of such sequences on any of the higher regular cardinals is not a consequence of the usual axioms of set theory. It is also known (see 88, that these principles hold in all currently known core models of set theory and that the global failures of these principles is of a considerable large cardinal strength. In particular, it is known that the failure of $\square_{\kappa}$ for strong limit singular $\kappa$ or the simultaneous failure of the $\square(\theta)$-principles have some important structural consequences most interesting of which is the principle of Projective Determinacy, $\mathrm{PD}$, stating that all projective games are determined (see [99], [55, 89]).

\section{Part 1. Combinatorial Compactness}

We start Part I with a well known large cardinal axiom that has played a crucial role in the development of set theory. Its influence on the cardinal arithmetic at singular cardinals and its influence on square sequences and consequently its nonrelativization to any of the current core models of set theory form a basis of the interest in this axiom. We give a precise list of these consequences since we intend to compare them with the corresponding consequences of combinatorial set theoretic principles that we introduce in later parts of this article.

Definition 0.1 ([27). An infinite cardinal $\kappa$ is compact ${ }^{2}$ if every set $S$ of sentences of $L_{\kappa \kappa}$ that has no model contains a subset $S_{0}$ of cardinality $<\kappa$ without a model ${ }^{3}$

Remark 0.2. Since $\omega$ is a compact cardinal, in what follows compact cardinal is usually required to be uncountable.

Here are some sample results that use this notion and which range over important themes of research of modern set theory. They serve as prototype themes for many results to be discussed in later sections of this article.

Theorem 0.3 (97]). Let $\kappa_{0}$ be the first uncountable compact cardinal. Then

(1) $\left(\forall \theta \geq \kappa_{0}\right) \theta^{\mathrm{cf} \theta}=\theta^{+} \cdot 2^{\mathrm{cf} \theta}{ }^{4}$

(2) $\square_{\kappa}$ fails for every $\kappa \geq \kappa_{0}$.

Theorem 0.4 ([89]). If there is a compact cardinal then all projective games are determined.

\footnotetext{
${ }^{1}$ In hindsight one can say that $\square\left(\omega_{1}\right)$ and $\square_{\omega}$ are directly responsible for the 'incompactness at the level of the first uncountable cardinal' alluded to above at the beginning of this Introduction (see 128, for more details).

${ }^{2}$ Or strongly compact in the more recent literature.

${ }^{3}$ Recall that in sentences of $L_{\kappa \lambda}$ we allow conjucntions and disjunctions of length $<\kappa$ and strings of quantifiers of length $<\lambda$.

4 The statement $(\forall \theta) \theta^{\operatorname{cf} \theta}=\theta^{+} \cdot 2^{\operatorname{cf} \theta}$ is the well-known Singular Cardinals Hypothesis, SCH, So this is saying that the Singular Cardinals Hypothesis holds above the first uncountable compact cardinal.
} 
Question 0.5. Is the compactness of the logic $L_{\omega_{1} \omega}$ as strong? More precisely, does the existence of an (uncountable) cardinal $\kappa$ for which the logic $L_{\omega_{1} \omega}$ is $\kappa$-compact $t^{5}$ has consequences as these appearing in Theorems 0.3 and 0.4 above?

We shall see below that the answer is positive but we shall be particularly interested in finding an optimal answer to the following variant of this question.

Question 0.6. What are the weak forms of compactness of this sort with as strong structural consequences on the universe of set theory?

\section{Compactness Principles for Graphs}

Recall that a graph is a structure of the form $\mathcal{G}=(X, E)$, where $X$ is a set, called a set of vertices of $\mathcal{G}$, and where $E$ is a symmetric irreflexive binary relation of $X$ called the set of edges of $\mathcal{G}$.

Example 1.1. The complete graph on the set of vertices $Z$ is the graph $\mathcal{K}_{Z}=$ $\left(Z, Z^{2} \backslash \triangle\right)$ and the discrete graph with vertex-set $Z$ is the graph $\mathcal{D}_{Z}=(Z, \emptyset)$.

Given two graphs $\mathcal{G}=(X, E)$ and $\mathcal{H}=(Y, F)$, a mapping $f: X \rightarrow Y$ is a homomorphism from $\mathcal{G}$ into $\mathcal{H}$ whenever

$$
(x, y) \in E \text { implies }(f(x), f(y)) \in F
$$

for all $x, y \in X$. We use the notation $\mathcal{G} \leq \mathcal{H}$ to denote the fact that there is a homomorphism from $\mathcal{G}$ to $\mathcal{H}$. If the vertex sets $X$ and $Y$ are equipped with topologies and measures then we use the notation $\mathcal{G} \leq_{C} \mathcal{H}, \mathcal{G} \leq_{B} \mathcal{H}$ or $\mathcal{G} \leq_{L} \mathcal{H}$ to record the fact that there is a homomorphism from $\mathcal{G}$ into $\mathcal{H}$ which is continuous, Borel or Baire measurable, or Lebesgue measurable, respectively.

Definition 1.2. The chromatic number of a graph $\mathcal{G}$ is defined by

$$
\operatorname{Chr}(\mathcal{G})=\min \left\{|Z|: \mathcal{G} \leq \mathcal{K}_{Z}\right\} .
$$

When the homomorphisms into $\mathcal{K}_{Z}$ are to be witnessed by continuous, Borel, Baire, or Lebesgue measurable maps, we use the notations $\operatorname{Chr}_{C}(\mathcal{G}), \operatorname{Chr}_{B}(\mathcal{G}), \operatorname{Chr}_{L}(\mathcal{G})$, respectively, for the corresponding variations on the chromatic number.

Remark 1.3. Thus, a graph $\mathcal{G}$ has countable chromatic number, or is countably chromatic, if its set of vertices can be (colored) covered by countably many (colors) $\mathcal{G}$-discrete sets, i.e., sets of vertices which contain no edges.

Definition 1.4. Given a class $\mathcal{H}$ of graphs, let $\kappa_{\mathcal{H}}$ be the minimal cardinal $\kappa$ (provided it exists) such that every graph $G \in \mathcal{H}$ with

$$
\operatorname{Chr}(G)>\aleph_{0}
$$

has a subgraph $G_{0}$ of cardinality $<\kappa$ such that

$$
\operatorname{Chr}\left(G_{0}\right)>\aleph_{0} .
$$

Remark 1.5. Clearly, for every class $\mathcal{H}$ of graphs,

$$
\kappa_{\mathcal{H}} \leq \text { the first uncountable compact cardinal. }
$$

Problem 1.6. Classify the classes of graphs $\mathcal{H}$ that have small compactness number $\kappa_{\mathcal{H}}$. In particular, classify the of classes of graphs $\mathcal{H}$ such that $\kappa_{\mathcal{H}}=\aleph_{2}$.

\footnotetext{
${ }^{5}$ Or in other words, which has the property that every set of sentences of $L_{\omega_{1} \omega}$ which has no model has a subset of cardinality $<\kappa$ without a model.
} 
Note that $\aleph_{2}$ is indeed the first possible nontrivial such a value for $\kappa_{\mathcal{H}}$ and so we are naturally led to the following question.

Question 1.7. Given a class $\mathcal{H}_{0}$ of graphs with $\kappa_{\mathcal{H}_{0}}$ equal to the minimal possible value, can one find any interesting structure of the universe of sets above this cardinal?

\section{Classes of Graphs with Small Compactness Numbers}

Example 2.1. Let $\mathcal{G}$ be the class of of all graphs. Not much is known about the corresponding compactness number $\kappa_{\mathcal{G}}$.

Theorem $2.2([26]) . \kappa_{\mathcal{G}} \geq \beth_{\omega}$

Sketch of Proof: Let $\gamma$ be an ordinal, and $k \geq 2$ an integer. We define the graph ( $[\gamma]^{k}$, Shift) by setting $\left(\left\{\alpha_{0}, \ldots, \alpha_{k-1}\right\}_{<},\left\{\beta_{0}, \ldots, \beta_{k-1}\right\}_{<}\right) \in$ Shift if and only if

$$
\alpha_{0}<\alpha_{1}=\beta_{0}<\alpha_{2}=\beta_{1}<\cdots<\alpha_{k-1}=\beta_{k-2}<\beta_{k-1} .
$$

Claim 1. $\operatorname{Chr}\left([\gamma]^{k}\right.$, Shift $) \leq \aleph_{0}$ whenever $|\gamma| \leq \beth_{k-1}$.

Proof. Induction on $k$ but for the stronger statement $\operatorname{Chr}\left(\gamma^{k}\right.$, Shift $) \leq \aleph_{0}$.

Claim 2. $\operatorname{Chr}\left([\gamma]^{k}\right.$, Shift $)>\aleph_{0}$ whenever $\gamma \geq \beth_{k-1}^{+}$.

Proof. Use $\beth_{k-1}^{+} \rightarrow(k+1)_{\aleph_{0}}^{k}$.

Remark 2.3. The construction makes perfect sense for finite ordinals as well. Moreover, the shift graph can be associated to any hypergraph in place of the complete hypergraph $\left(\gamma,[\gamma]^{k}\right)$. Let us explain this in case $k=2$, i.e., in case of a directed or an undirected graph. Thus, given a graph $\mathcal{G}=(X, E)$ and a total ordering $<$ of its vertex set $X$, let

$$
\operatorname{Shift}(\mathcal{G})=\left(V_{\text {Shift }}, E_{\text {Shift }}\right)
$$

be the graph whose vertex set $V_{\text {Shift }}$ is equal to the set $E$ of edges of $\mathcal{G}$ and where we put $\left((x, y),\left(x^{\prime}, y^{\prime}\right)\right)$ in the edge relation $E_{\text {Shift }}$ of $\operatorname{Shift}(\mathcal{G})$ whenever $x<y=x^{\prime}<y^{\prime}$. Then we have the following fact proved in a similar manner as above.

Theorem $2.4([26]) \cdot \operatorname{Chr}(\mathcal{G})>2^{\kappa}$ implies $\operatorname{Chr}(\operatorname{Shift}(\mathcal{G}))>\kappa$.

\section{Dilworth's Theorem and Chromatic Numbers of Incomparability GRAPHS}

In this section we investigate the class $\mathcal{H}_{D}$ of graphs whose vertex sets are posets (or, more generally, quasi-ordered sets) and whose edges are pairs of incomparable points. The following famous result of Dilworth is giving us the compactness number for families of $k$-chromatic such graphs, where $k$ is some fixed finite number.

Theorem 3.1 ([21]). For a finite integer $k \geq 1$, a poset $\mathcal{P}$ can be decomposed into $\leq k$ chains iff every subposet $\mathcal{P}_{0} \subseteq \mathcal{P}$ of cardinality $\leq k+1$ can be decomposed into $\leq k$ chains ${ }^{6}$

It is therefore natural to investigate the compactness number of the family of all countably chromatic incomparability graphs on posets.

${ }^{6}$ In other words, a poset can be covered by $\leq k$ chains iff it contains no antichain of size $k+1$. 
Definition 3.2. Let $\kappa_{D}$ be the minimal cardinal $\kappa$ (if it exists) with the property that a partially ordered set $\mathcal{P}$ can be decomposed into countably many chains if every subordering $\mathcal{P}_{0} \subseteq \mathcal{P}$ of size $<\kappa$ can be decomposed into countably many chains.

Conjecture 3.3 (Galvin's Conjecture). $\aleph_{2}$ is a possible value of $\kappa_{D}$.

While Galvin's conjecture is still an open problem the following result verifies its correctness for an important special case.

Theorem 3.4 ([47]). Galvin's Conjecture holds in the class of Borel quasi-ordered sets defined on Polish spaces 7

\section{Rado's Theorem and Chromatic Numbers of Interval Graphs}

In this section we investigate a special kind of graphs, the class $\mathcal{H}_{R}$ of graphs of the form $(I, E)$, where $I$ is some family of nonempty intervals, or more generally, convex sets of some linearly ordered set $L$ and where two intervals form an edge if their intersection is nonempty. The following result of Richard Rado computes the compactness numbers for each of the families $\left\{(I, E) \in \mathcal{H}_{R}: \operatorname{Chr}(I, E) \leq k\right\}$ for some finite number $k$.

Theorem 4.1 (83]). For an integer $k \geq 1$, a family $\mathcal{F}$ of intervals of some linearly ordered set $\left(L,<_{L}\right)$ can be decomposed into $\leq k$ subfamilies of pairwise disjoint intervals if this is true about any subfamily $\mathcal{F}_{0} \subseteq \mathcal{F}$ of size $\leq k+1$.

It is therefore quite natural to investigate the compactness number of countably chromatic intersection graphs on intervals.

Definition 4.2. Let $\kappa_{R}$ be the minimal cardinal $\kappa$ (if it exists) with the property that a family $\mathcal{F}$ of intervals of some linearly ordered set $\left(L,<_{L}\right)$ can be decomposed into countably many subfamilies of pairwise disjoint intervals if this is true about every subfamily $\mathcal{F}_{0} \subseteq \mathcal{F}$ of cardinality $<\kappa$.

Conjecture 4.3 (84]). [Rado's Conjecture] $\kappa_{R}=\aleph_{2}$.

Theorem 4.4 ([114]). Rado's Conjecture is equivalent to the statement that an arbitrary tree $\mathcal{T}$ can be decomposed into countably many antichains if and only if every subtree $\mathcal{T}_{0}$ of $\mathcal{T}$ of cardinality at most $\aleph_{1}$ allows such a decomposition.

Corollary 4.5. $\kappa_{R} \leq \kappa_{D}$, and so in particular, Rado's Conjecture is a consequence of Galvin's Conjecture.

Proof. Consider a tree $\mathcal{T}=\left(T, \leq_{T}\right)$ and pick a well-ordering $<_{w}$ of its domain $T$. Define another ordering $\leq_{P}$ on $T$ by letting $s \leq_{P} t$ if and only if $s=t$ or $s$ and $t$ are $\leq_{T}$-incomparable and $u(s)<_{w} u(t)$, where $u(s)$ and $u(t)$ are the immediate successors of the node $v=s \wedge t$ extended by $s$ and $t$, respectively. Then $\mathcal{P}=\left(T, \leq_{T}\right)$ is a partially ordered set living on the same domain $T$ such that

$$
(\forall C \subseteq T) C \text { is a chain of } \mathcal{P} \text { iff } C \text { is an antichain of } \mathcal{T} \text {. }
$$

So if the tree $\mathcal{T}$ cannot be covered by countably many antichains, the corresponding poset $\mathcal{P}$ is not the union of countably many chains, so there is $T_{0} \subseteq T$ of size $<\kappa_{D}$ such that $\mathcal{P}\left\lceil T_{0}\right.$ and $\mathcal{T} \uparrow T$ have the same corresponding properties. This shows that $\kappa_{D} \geq \kappa_{R}$.

\footnotetext{
${ }^{7}$ More precisely, if a Borel quasi-ordered set contains no perfect antichain then it can be decomposed into countably many Borel chains.
} 
Remark 4.6. In fact in can be shown that Galvin's Conjecture for the class of finite-dimensiona 8 posets is equivalent to Rado's Conjecture.

Problem 4.7. Is $\kappa_{R}$ a regular cardinal? If not, can it have countable cofinality?

Theorem 4.8 ([103]). The equality $\kappa_{R}=\aleph_{2}$ is consistent relative to the consistency of the existence of a compact uncountable cardinal.

We repeat that the consistency of Galvin's Conjecture is still an open problem.

The formulation of Rado's Conjecture in terms of trees is a key step behind basically all applications of this principle that appeared afterwards. The reason for this perhaps lies in the fact that trees that are not countably chromatic $9^{9}$ behave much like the first uncountable ordinal $\omega_{1}$. The following two facts are illustrations of this phenomenon.

Lemma 4.9 ([101]). [Pressing-Down Lemma for Trees] For every non-special tree $T$ and every regressive ${ }^{10}$ mapping $f: T \rightarrow T$ there is a non-special subtree $S$ of $T$ on which $f$ is constant.

Lemma 4.10 ([101]). [ $\Delta$-System Lemma for Trees] For every family $F_{t}(t \in T)$ of finite sets indexed by a non-special tree $T$ there is a finite set $R$ and a non-special subtree $U$ of $T$ such that $F_{s} \cap F_{t}=R$ whenever $s, t \in U$ are chosen such that $s<_{T} t$.

\section{The Set-Theoretic Universe Above Rado's Cardinal}

Theorem 5.1 ([114]). The Singular Cardinals Hypothesis holds above $\kappa_{R}$. More precisely,

(1) $\theta^{\lambda}=\theta$ for regular $\theta, \lambda$ such that $\theta \geq\left(\kappa_{R}\right)^{\lambda}$.

(2) $\theta^{\text {cf } \theta}=\theta^{+} \cdot 2^{\operatorname{cf} \theta}$ for every $\theta \geq\left(\kappa_{R}\right)^{\bar{\aleph}_{0}}$.

Proof Sketch. The essential difficulty is in proving the equality $\theta^{\aleph_{0}}=\theta$ by induction on regular cardinals $\theta \geq\left(\kappa_{R}\right)^{\aleph_{0}}$. By Silver's theorem (see [92]) we can afford to concentrate on the case

$$
\theta=\kappa^{+} \text {for some } \kappa \geq\left(\kappa_{R}\right)^{\aleph_{0}} \text { with } \operatorname{cf}(\kappa)=\omega .
$$

Choose a strictly increasing converging sequence $\kappa_{i} \nearrow \kappa$ consisting of regular cardinals $\geq\left(\kappa_{R}\right)^{\aleph_{0}}$. For $a, b \in \prod_{i<\omega} \kappa_{i}$ set:

$$
\begin{array}{cc}
a<^{*} b \text { iff }\left(\forall^{\infty} i\right) a(i)<b(i), & a \leq^{*} b \text { iff }\left(\forall^{\infty} i\right) a(i) \leq b(i), \\
a<b \text { iff }(\forall i) a(i)<b(i), & a \leq b \text { iff }(\forall i) a(i) \leq b(i) .
\end{array}
$$

Choose $A=\left\{a_{\xi}: \xi<\kappa^{+}\right\} \subseteq \prod_{i<\omega} \kappa_{i}$ such that

(1) $\xi<\eta$ implies $a_{\xi} \leq^{*} a_{\eta}$,

(2) $\forall \xi(\exists \eta>\xi) a_{\xi}<^{*} a_{\eta}$

(3) $A$ is closed under finite changes of its elements.

Choose $\tau:\left[\kappa^{+}\right]^{2} \rightarrow \omega$ such that

\footnotetext{
${ }^{8}$ Recall, that the dimension of a poset $\left(P, \leq_{P}\right)$ is the minimal cardinality of a family $\leq_{i}(i \in I)$ of linear orderings of the set $P$ such that $\leq_{P}=\bigcap_{i \in I} \leq_{i}$.

${ }^{9}$ More precisely, trees whose comparability graphs are not countably chromatic, or in other words, trees that cannot be decomposed into countably many antichains. Such trees are known in the literature under the name of non-special trees.

${ }^{10}$ We say that a mapping $f: T \rightarrow T$ defined on a tree $T$ is regressive if $f(t)<_{T} t$ for all non-minimal $t \in T$.
} 
(4) $\tau(\alpha, \gamma) \leq \max \{\tau(\alpha, \beta), \tau(\beta, \gamma)\}$ whenever $\alpha<\beta<\gamma$,

(5) for all $\alpha<\kappa^{+}$and $n<\omega$, the set $\{\xi<\alpha: \tau(\xi, \alpha) \leq n\}$ has size $\leq \kappa_{n}$.

For a subset $t$ of $A$, let $\sup (t) \in \prod_{i<\omega} \kappa_{i}$ be defined by $\sup (t)(i)=\sup _{a \in t} a(i)$.

For $\alpha<\kappa^{+}$and $i<\omega$, set

$$
A_{i}(\alpha)=\left\{a_{\xi}: \xi<\alpha \& \tau(\xi, \alpha) \leq i\right\}
$$

and

$$
A_{i}^{*}(\alpha)=\left\{\sup (t): t \in\left[A_{i}(\alpha)\right]^{\leq \aleph_{0}}\right\} .
$$

By the inductive hypothesis $\left|A_{i}^{*}(\alpha)\right| \leq \kappa_{i}$ for all $\alpha<\theta=\kappa^{+}$and $i<\omega$. Let

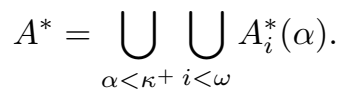

Then $\left|A^{*}\right| \leq \theta$.

Let $\mathcal{T}_{A}$ be the set of all <-increasing countable sequences $t=\left\{t_{\xi}: \xi \leq \mu\right\}$ of successor length, such that

$$
\sup _{\xi<\lambda} t_{\xi} \notin A^{*} \text { for all limit ordinals } \lambda \leq \mu .
$$

We consider $\mathcal{T}_{A}$ as a tree ordered by end-extension and choose a lexicographical ordering on $\mathcal{T}_{A}$ and let $\mathcal{L}_{A}$ be the corresponding linear ordering. Finally, let

$$
\mathcal{F}_{A}=\left\{\left\{s \in \mathcal{T}_{A}: t \sqsubseteq s\right\}: t \in \mathcal{T}_{A}\right\},
$$

a family of intervals of $\mathcal{L}_{A}$.

Claim 1. Every subfamily of $\mathcal{F}_{A}$ of cardinality $\leq \kappa$ can be decomposed into countably many subfamilies of pairwise disjoint intervals.

Claim 2. If $\mathcal{F}_{A}$ itself can be decomposed into countably many subfamilies of pairwise disjoint intervals there is a Namba subtre ${ }^{11} W \subseteq \bigcup_{k<\omega} \prod_{i<k} \kappa_{i}$ such that the set $[W]$ of all of its infinite branches is included in $A^{*}$. So, in particular, $\kappa^{\aleph_{0}} \leq \kappa^{+}$.

These claims complete the proof of Theorem 5.1

Recall that a $C$-sequence on an ordinal $\theta$ is simply a sequence $C_{\alpha}(\alpha<\theta)$ of sets, where for each $\alpha<\theta$, the set $C_{\alpha}$ is closed and unbounded in $\alpha$. We say that a $C$-sequence $C_{\alpha}(\alpha<\theta)$ is coherent if

$$
C_{\alpha}=C_{\beta} \cap \alpha \text { whenever } \alpha \text { is a limit point of } C_{\beta} .
$$

Clearly, the sequence $C_{\alpha}=\alpha(\alpha<\theta)$ is coherent so we are interested in less trivial examples. Thus, we say that a $C$-sequence $C_{\alpha}(\alpha<\theta)$ is trivial if there is a closed and unbounded set $C \subseteq \theta$ such that

$$
C_{\alpha}=C \cap \alpha \text { whenever } \alpha \text { is a limit point of } C \text {. }
$$

Let $\square(\theta)$ denote the statement that $\theta$ supports a nontrivial coherent $C$-sequence $C_{\alpha}(\alpha<\theta)$. Note that if $\theta=\kappa^{+}$then every $\square_{\kappa}$-sequence in the sense of [54] is a $\square(\theta)$-sequence but in general $\square\left(\kappa^{+}\right)$does not imply $\square_{\kappa}$.

Theorem 5.2 ([114]). $\square(\theta)$ fails for all ordinals $\theta$ of cofinality $\geq \kappa_{R}$.

\footnotetext{
${ }^{11}$ Here, we consider $T=\bigcup_{k<\omega} \prod_{i<k} \kappa_{i}$ as a tree ordered by extension. A subtree $S$ of $T$ is a Namba subtree if it is downwards closed and if it preserves the degrees of nodes, i.e., if $\left|\left\{\xi<\kappa_{i}: t^{\urcorner} \xi \in S\right\}\right|=\kappa_{i}$ for every $t \in S$ of length $i$.
} 
Sketch of Proof: Note that it suffices to prove this for regular cardinals $\theta \geq \kappa_{R}$. Towards a contradiction suppose we have a nontrivial coherent sequence $C_{\alpha}(\alpha<\theta)$. Consider the tree $\mathcal{T}$ of all countable closed subsets $A$ of $\theta$ such that

$$
\sup \left(A \cap C_{\alpha}\right)<\alpha \text { for all } \alpha \in A
$$

with the end-extension as the tree-ordering. Then $\mathcal{T}$ is a tree that cannot be decomposed into countably many antichains though for all $\delta<\theta$, the subtree $\mathcal{T}\lceil\delta=\{A \in \mathcal{T}: \max (A)<\delta\}$ does have such a decomposition. To see this, define $f: \mathcal{T}\lceil\delta \rightarrow \mathcal{T}\lceil\delta$, by

$$
f(A)=A \cap(\xi+1) \text { where } \xi=\max \left(A \cap C_{\delta} .\right)
$$

Then $f$ is a regressive mapping with the property that for every $B \in \mathcal{T} \uparrow \delta$, the pre-image $f^{-1}(B)$ can be decomposed into countably many antichains.

This shows that the assumption $\kappa_{R}<\infty$ that there is a Rado cardinal has considerable large-cardinal strength. Using this and some deep work about inner models of set theory and determinacy one can state the following kind of direct consequences.

Theorem 5.3 ([55]). If there is a Rado cardinal then projective determinacy holds.

Corollary 5.4 ([55]). RC implies PD.

We finish this section with another direct consequences of the existence of Rado's cardinal. It can be seen as a corollary of the proof of Theorem 8.18 below.

Theorem 5.5. $\theta \rightarrow\left(\omega_{1}\right)_{\omega_{1}, \omega}^{<\omega}$ for every regular $\theta \geq \kappa_{R}{ }^{12}$

\section{Rado's Conjecture and the Continuum}

Theorem 6.1 (114]). Rado's Conjecture implies $\mathfrak{c} \leq \aleph_{2}{ }^{13}$

Proof. Fix an $e:\left[\omega_{2}\right]^{2} \rightarrow \omega_{1}$ such that $e(\alpha, \gamma) \neq e(\beta, \gamma)$ for $\alpha<\beta<\gamma<\omega_{2}$.

Let $\mathcal{C}_{e}$ be the collection of all $A \in\left[\omega_{2}\right]^{\aleph_{0}}$ such that

(1) $A \cap \omega_{1} \in \omega_{1}$

(2) $\alpha \in A \Longleftrightarrow \alpha+1 \in A$

(3) $\alpha<\beta$ in $A \Rightarrow e(\alpha, \beta) \in A$

(4) $\nu=e(\alpha, \beta) \& \nu, \beta \in A \Rightarrow \alpha \in A$.

Define

$$
\mathcal{S}_{e}=\left\{A \in \mathcal{C}_{e}:\left(\forall \nu<\omega_{1}\right)\left(\forall \alpha<\omega_{2}\right) A \neq\{\xi<\alpha: e(\xi, \alpha)<\nu\}\right\} .
$$

Note 6.2. If $A \varsubsetneqq B$ in $\mathcal{C}_{e}$ and $A \cap \omega_{1}=B \cap \omega_{1}$ then $A \notin \mathcal{S}_{e}$.

Note 6.3. $\mathcal{S}_{e} \cap[Y]^{\aleph_{0}}$ is not stationary in $[Y]^{\aleph_{0}}$ for every uncountable $Y \subseteq \omega_{2}$.

Let $\mathcal{T}_{e}$ be the tree of all countable continuous chains of elements of $\mathcal{S}_{e}$ whose unions are also elements of $\mathcal{S}_{e}$. Let $\mathcal{L}_{e}$ be the linear ordering obtained by lexicographically ordering $\mathcal{T}_{e}$. For $t \in \mathcal{T}_{e}$, let

$$
I_{t}=\left\{s \in \mathcal{T}_{e}: t \sqsubseteq s\right\},
$$

\footnotetext{
${ }^{12}$ Recall that $\theta \rightarrow\left(\omega_{1}\right)_{\omega_{1}, \omega}^{<\omega}$ means that for every mapping $F: \theta^{<\omega} \rightarrow \omega_{1}$ there exists an uncountable set $X \subseteq \theta$ such that $\left|f^{\prime \prime} X^{<\omega}\right| \leq \aleph_{0}$.

${ }^{13}$ It is known that Rado's Conjecture does not decide whether $\mathfrak{c}=\aleph_{1}$ or $\mathfrak{c}=\aleph_{2}$ (see [103]).
} 
a convex subset of the linearly ordered set $\mathcal{L}_{e}$. Let

$$
\mathcal{F}_{e}=\left\{I_{t}: t \in \mathcal{T}_{e}\right\}
$$

be a family of convex subsets of $\mathcal{L}_{e}$. For $\delta<\omega_{2}$, let

$$
\mathcal{F}_{e}^{\delta}=\left\{I_{t} \in \mathcal{F}_{e}: \bigcup t \subseteq \delta\right\}
$$

Claim 1. For each $\delta<\omega_{2}$ the family $\mathcal{F}_{e}^{\delta}$ can be decomposed into countably many subfamilies consisting of pairwise disjoint intervals. So in particular, every subfamily of $\mathcal{F}_{e}$ of cardinality $<\aleph_{2}$ can be decomposed into countably many subfamilies consisting of pairwise disjoint intervals.

Claim 2. If $\mathcal{F}_{e}$ itself can be decomposed into countably many subfamilies consisting of pairwise disjoint intervals, then $\mathbb{R} \subseteq L[e]$, so in particular $\mathfrak{c} \leq \aleph_{2}$.

Proof. Note that if $\mathbb{R} \nsubseteq L[e]$ then the set $\mathcal{S}_{e}$ is a stationary subset of $\left[\omega_{2}\right]^{\aleph_{0}}$.

This completes the proof of Theorem 6.1 .

Theorem 6.4 ([114]). Rado's Conjecture implies that every transitive model of a sufficiently large fragment of ZFC which correctly computes $\aleph_{2}$ must contain all the reals.

Proof. Suppose $\mathbb{R} \nsubseteq M$. Then $\mathcal{S}=\left[\omega_{2}\right]^{\aleph_{0}} \backslash M$ is a stationary subset of $\left[\omega_{2}\right]^{\aleph_{0}}$ (see [10] and [44]). Choose $f:\left[\omega_{2}\right]^{2} \rightarrow \omega_{1}$ such that $f \in M$ and $f(\alpha, \gamma) \neq f(\beta, \gamma)$ whenever $\alpha<\beta<\gamma<\omega_{2}$. Then $\mathcal{S} \cap \mathcal{C}_{e} \subseteq \mathcal{S}_{e}$. Then $\mathcal{S}_{e}$ is stationary and therefore Rado's Conjecture is false.

\section{Rado's Conjecture and Chang's Conjecture}

Recall that Chang's Conjecture, CC, is the statement that every structure

$$
\langle A, U, \ldots\rangle
$$

of countable signature where $A$ has cardinality $\aleph_{2}$ and where $U$ is a distinguished unary predicate of cardinality $\aleph_{1}$ has an elementary substructure

$$
\langle B, B \cap U, \ldots\rangle
$$

with $|B|=\aleph_{1}$ and $|B \cap U|=\aleph_{0}$. This was one of the first combinatorial model theoretic statements on the level of $\omega_{2}$ with a considerable large cardinal strength. In this section we give some explanations of the following result which points out the connection between $\mathrm{RC}$ and $\mathrm{CC}$.

Theorem 7.1 ([114]). Rado's Conjecture implies Chang's Conjecture.

Since the proof of this result has ideas of general interest, we give some details.

Definition 7.2. The Game $G_{\omega}\left(\omega_{2}, \omega_{1}\right)$ is played as follows

$$
\begin{array}{l|l|l|l|l|l}
\text { I } & f_{0}: \omega_{2} \rightarrow \omega_{1} & & f_{1}: \omega_{2} \rightarrow \omega_{1} & & \cdots \\
\hline \text { II } & & \delta_{0} & & \delta_{1} & \cdots
\end{array}
$$

where $\delta_{i}<\omega_{2}$ for all $i<\omega$. Player II wins $f_{0}, \delta_{0}, f_{1}, \delta_{1}, \ldots$ if

$$
\left\{\alpha<\omega_{2}: f_{n}(\alpha)<\sup _{i<\omega} \delta_{i} \text { for all } n<\omega\right\}
$$

is an unbounded subset of $\omega_{2}$; otherwise I wins. 
Theorem 7.3. Rado's Conjecture implies that II has a winning strategy in the game $G_{\omega}\left(\omega_{2}, \omega_{1}\right)$.

Proof. For $A \in\left[\omega_{1} \cup \omega_{1}^{\omega_{2}}\right]^{\aleph_{0}}$, let

$$
D_{A}=\left\{\alpha<\omega_{2}:(\forall f \in A) f(\alpha) \in A\right\} .
$$

Let

$$
\mathcal{S}_{G}=\left\{A \in\left[\omega_{1} \cup \omega_{1}^{\omega_{2}}\right]^{\aleph_{0}}: A \cap \omega_{1} \in \omega_{1} \& \sup D_{A}<\omega_{2}\right\} .
$$

Claim 1. II has a winning strategy in $G_{\omega}\left(\omega_{2}, \omega_{1}\right)$ if and only if $\mathcal{S}_{G}$ is not a stationary subset of $\left[\omega_{1} \cup \omega_{1}^{\omega_{2}}\right]^{\aleph_{0}}$.

Definition 7.4. For $A, B \in \mathcal{S}_{G}$ we say $B$ strongly includes $A$ if

$$
A \subseteq B \text { and } A \cap \omega_{1}<B \cap \omega_{1} .
$$

Let $\mathcal{T}_{G}$ be the tree of all countable continuous strongly increasing chains $t$ of elements of $\mathcal{S}_{G}$ such that $\bigcup t \in \mathcal{S}_{G}$.

Let $\mathcal{L}_{G}$ be the linearly ordered set obtained by lexicographically ordering the tree $\mathcal{T}_{G}$. For $t \in \mathcal{T}_{G}$, let

$$
I_{t}=\left\{s \in \mathcal{T}_{G}: t \sqsubseteq s\right\},
$$

a convex set (interval) of the linearly ordered set $\mathcal{L}_{G}$. Let

$$
\mathcal{F}_{G}=\left\{I_{t}: t \in \mathcal{T}_{G}\right\} .
$$

Claim 1. Every subfamily $\mathcal{F}_{0}$ of $\mathcal{F}_{G}$ of cardinality $<\aleph_{2}$ can be decomposed into countably many subfamilies of pairwise disjoint intervals.

Claim 2. If $\mathcal{F}_{G}$ itself can be decomposed into countably many subfamilies of pairwise disjoint intervals then $\mathcal{S}_{G}$ is nonstationary and therefore II has a winning strategy in the game $G_{\omega}\left(\omega_{2}, \omega_{1}\right)$.

This completes the proof of Theorem 7.3 .

Lemma 7.5. If II has a winning strategy in $G_{\omega}\left(\omega_{2}, \omega_{1}\right)$ then for every countable

$$
M \prec\left(H_{\theta}, \in,<\right),
$$

for $\theta$ a sufficiently large regular cardinal, there is

$$
M^{*} \prec\left(H_{\theta}, \in,<\right)
$$

such that $M \subseteq M^{*}, M^{*} \cap \omega_{1}=M \cap \omega_{1}$, and $M \cap \omega_{2} \neq M^{*} \cap \omega_{2}$.

Proof. Take $M^{*}=\operatorname{Sk}(M \cup\{\alpha\})$ for $\alpha \in D_{M \cap\left(\omega_{1} \cup \omega_{1}^{\omega_{2}}\right)}$ with $\alpha>\sup \left(M \cap \omega_{2}\right){ }^{14}$

Note that this finishes the proof of Theorem 7.1. The conclusion of Lemma 7.5 is a strong form of Chang's Conjecture which we denote by $\mathrm{CC}^{*}$. This way of strengthening CC showed up first during an analysis of conditions that guarantee the semi-properness of the standard Namba forcing which changes the cofinality of $\omega_{2}$ to $\omega$ (see 90]; pp. 355-400). The difference between CC and its strong form $\mathrm{CC}^{*}$ can be seen from the following result.

Theorem 7.6 ([114]). CC* implies $\mathfrak{c} \leq \aleph_{2}$.

\footnotetext{
${ }^{14}$ We are using here the functor $A \mapsto D_{A}$ from the proof of Theorem 7.3 above.
} 
Recall that CC has no influence on the cardinality of the continuum since it is preserved by forcing extensions over posets satisfying the countable chain condition.

Since the proof of Theorem 7.6 involves interesting ideas we give its sketch. First of all we note the following fact.

Lemma 7.7 ([114]). $\mathrm{CC}^{*}$ implies that for every stationary $S \subseteq\left[\omega_{2}\right]^{\aleph_{0}}$ there exists uncountable $\alpha<\omega_{2}$ such that $S \cap[\alpha]^{\aleph_{0}}$ is stationary in $[\alpha]^{\aleph_{0}}$.

Proof. Suppose $S \cap[\alpha]^{\aleph_{0}}$ is not stationary whenever $\omega_{1} \leq \alpha<\omega_{2}$, and for each such $\alpha$ choose one-to-one $e_{\alpha}: \alpha \rightarrow \omega_{1}$ such that $A_{\nu}(\alpha)=\left\{\xi<\alpha: e_{\alpha}(\xi)<\nu\right\} \notin S$ for all non-zero limit ordinals $\nu<\omega_{1}$. Since $S$ is stationary, for every sufficiently large regular cardinal $\theta$, we can choose a countable $M \prec\left(H_{\theta}, \in,<\right)$ such that $M \cap \omega_{2} \in S$. Let $M^{*} \prec\left(H_{\theta}, \in,<\right)$ be an $\omega_{2}$-end-extension of $M$ guaranteed by $\mathrm{CC}^{*}$ and let $\alpha=\min \left(\left(M^{*} \cap \omega_{2}\right) \backslash M\right)$. Then $A_{\nu}(\alpha)=M \cap \omega_{2} \in S$ for $\nu=M \cap \omega_{1}=M^{*} \cap \omega_{1}$, a contradiction.

The conclusion of Lemma 7.7 is known in the literature under the name of Weak Reflection Principle for $\omega_{2}, \operatorname{WRP}\left(\omega_{2}\right)[15$ Thus we have established the following

$$
\mathrm{RC} \Rightarrow \mathrm{CC}^{*} \Rightarrow \operatorname{WRP}\left(\omega_{2}\right) \text {. }
$$

So it remains to establish the following.

Theorem 7.8 ([105]). $\operatorname{WRP}\left(\omega_{2}\right)$ implies $\mathfrak{c} \leq \aleph_{2}$.

Proof. Since every closed and unbounded subset of $\left[\omega_{2}\right]^{\aleph_{0}}$ has cardinality at least continuum (see [10]), if $\mathfrak{c}>\aleph_{2}$, the set $S=\left[\omega_{2}\right]^{\aleph_{0}} \backslash\left\{A_{\nu}(\alpha): \alpha<\omega_{2}, \nu<\omega_{1}\right\}$ is stationary. But clearly $S \cap[\alpha]^{\aleph_{0}}$ is not stationary in $[\alpha]^{\aleph_{0}}$ for all $\alpha<\omega_{2}$.

In 91 this result was extended to all other regular cardinals $\theta$ in place of $\omega_{2}{ }^{16}$ which reiterates the following natural question.

Question 7.9 (114]). Does RC imply that $\operatorname{WRP}(\theta)$ for all cardinals $\theta \geq \omega_{2}$ ?

It turns out that $\mathrm{CC}^{*}$ is the $\omega_{2}$-case of a general principle isolated in [23] as one of the equivalents of the well-known assertion that forcing notions that preserve stationary subsets of $\omega_{1}$ are semi-proper, a principle that has played an important role in the original proof of the consistency of Martin's Maximum in [39].

Definition 7.10. For $\lambda \geq \omega_{2}$, let $\operatorname{CC}^{*}(\lambda)$ be the statement that for an arbitrary regular cardinal $\theta>\lambda$, a well-ordering $<_{w}$ of $H_{\theta}$, a set $a \in[\lambda]^{\omega_{1}}$, and a countable $M \prec\left(H_{\theta}, \in,<_{w}\right)$ there is countable $M^{*} \prec\left(H_{\theta}, \in,<_{w}\right)$ and $b \in[\lambda]^{\omega_{1}} \cap M^{*}$ such that $b \supseteq a, M^{*} \supseteq M$ and $M^{*} \cap \omega_{1}=M \cap \omega_{1}$. Let $\mathrm{CC}^{* *}$ be the statement that $\mathrm{CC}^{*}(\lambda)$ holds for all $\lambda \geq \omega_{2}$.

A proof structured very much like the above proof that $\mathrm{RC}$ implies $\mathrm{CC}^{*}$ will give us the following more general fact.

Theorem 7.11 ([22]). Rado's Conjecture implies $\mathrm{CC}^{* *}$.

\footnotetext{
${ }^{15}$ More generally, for a cardinal $\theta>\omega_{1}$, we let $\operatorname{WRP}(\theta)$ be the principle saying that for every stationary set $S \subseteq[\theta]^{\aleph_{0}}$ and every set $\omega_{1} \subseteq X \subseteq \theta$ of cardinality $\aleph_{1}$ there is a subset $X \subseteq Y \subseteq \theta$ also of cardinality $\aleph_{1}$ such that the intersection $S \cap[Y]^{\aleph_{0}}$ is stationary in $[Y]^{\aleph_{0}}$ (see [39, [137).

${ }^{16}$ More precisely, 91] establishes that $\operatorname{WRP}(\theta)$ implies $\theta^{\aleph_{0}}=\theta$ for regular cardinals $\theta \geq \omega_{2}$.
} 
So, in particular (see [39], 23]), Rado's Conjecture implies that ideal $\mathrm{NS}_{\omega_{1}}$ of all nonstationary subsets of $\omega_{1}$ is precipitous, i.e. that all its generic ultrapowers are well-founded. It turns out that this can be improved to show that Rado's Conjecture implies that the ideal $\mathrm{NS}_{\omega_{1}}$ is in fact presaturated, another well-known statement with a considerable large cardinal strength. We recall the definition.

Definition 7.12. We say that the ideal $\mathrm{NS}_{\omega_{1}}$ of non-stationary subsets of $\omega_{1}$ is presaturated if for every sequence $\mathcal{A}_{n} \quad(n<\omega)$ of maximal antichains of the quotient algebra $\mathcal{P}\left(\omega_{1}\right) / \mathrm{NS}_{\omega_{1}}$ and every stationary $S \subseteq \omega_{1}$ there is stationary $T \subseteq S$ such that $\left|\left\{A \in \mathcal{A}_{n}: A \cap T \notin \mathrm{NS}_{\omega_{1}}\right\}\right| \leq \aleph_{1}$ for all $n<\omega$.

Theorem 7.13 (36]). Rado's Conjecture implies that the ideal $\mathrm{NS}_{\omega_{1}}$ of nonstationary subsets of $\omega_{1}$ is presaturated.

We finish this section with a natural question.

Question 7.14. Does the Strong Chang Conjecture $\mathrm{CC}^{* *}$ imply the Singular Cardinals Hypothesis?

\section{An Application of Rado's Conjecture to Erdős-Hajnal Graphs}

To every cardinal $\theta$ we associate the Erdős-Hajnal graph

$$
\operatorname{EH}(\theta)=\left(\omega^{\theta}, \perp\right),
$$

where we set $f \perp g$ iff $\{\alpha<\theta: f(\alpha)=g(\alpha)\}$ is bounded in $\theta$. Similarly one defines the other versions of the graph:

$$
\operatorname{EH}\left(\lambda^{\theta}\right)=\left(\lambda^{\theta}, \perp\right) .
$$

Thus, $\operatorname{EH}(\theta)=\operatorname{EH}\left(\omega^{\theta}\right)$. The following fact shows that if the graph $\operatorname{EH}(\theta)$ is uncountably chromatic than $\theta$ must be smaller than the compactness number $\kappa_{\mathcal{G}}$ for the class $\mathcal{G}$ of all graphs.

Theorem 8.1 ([26]). If $\theta$ is regular then every subgraph of $\mathrm{EH}(\theta)$ of cardinality $<\theta$ is countably chromatic.

Proof. Given a subset $\mathcal{X}$ of $\omega^{\theta}$ of cardinality $<\theta$, find $\delta<\theta$ such that whenever $f, g \in \mathcal{X}$ are such that $f \perp g$ then $f(\gamma) \neq g(\gamma)$ for all $\gamma \geq \delta$. Then $f \mapsto f(\delta)$ is a good coloring on $\mathcal{X}$ showing that $(\mathcal{X}, \perp)$ is countably chromatic.

On the other hand, the following fact shows that $\operatorname{EH}(\theta)$ is likely to detect any other incompactness of the countable chromaticity at the level $\theta$.

Theorem $8.2([26])$. Suppose that a graph $G=\left(\theta, E_{G}\right)$ on the vertex set $\theta$ has the property that all of its restrictions $\operatorname{Chr}(G \uparrow \gamma)(\gamma<\theta)$ are countably chromatic. Then $G$ admits a homomorphism into $\mathrm{EH}(\theta)$. So in particular, $\operatorname{Chr}(\mathrm{EH}(\theta)) \geq$ $\operatorname{Chr}(G)$ for any such graph $G=\left(\theta, E_{G}\right)$.

Proof. For each $\gamma<\theta$ fix a coloring $g_{\gamma}: \gamma \rightarrow \omega$ witnessing $\operatorname{Chr}\left(\gamma, E_{G} \cap[\gamma]^{2}\right) \leq \aleph_{0}$ Define $\Phi: G \rightarrow \omega^{\theta}$ by setting

$$
\Phi(\alpha)(\gamma)= \begin{cases}0 & \text { if } \alpha \geq \gamma \\ g_{\gamma}(\alpha) & \text { if } \alpha<\gamma\end{cases}
$$

Then $\{\alpha, \beta\} \in E_{G} \Rightarrow \Phi(\alpha) \perp \Phi(\beta)$, i.e. $\Phi$ is a homomorphism. So if $G$ is of chromatic number $\geq \lambda$ then also $\operatorname{Chr}(\operatorname{EH}(\theta)) \geq \lambda$. 
Question $8.3(26])$. For which $\theta$ is $\operatorname{Chr}(\operatorname{EH}(\theta))>\aleph_{0}$ ?

Note that $\operatorname{Chr}(\operatorname{EH}(\omega))=2^{\aleph_{0}}$ and $\operatorname{Chr}\left(\operatorname{EH}\left(\omega_{1}\right)\right) \geq \aleph_{2}$, so the first interesting case of this question is the following.

Question 8.4 (26]). Is $\mathrm{EH}\left(\omega_{2}\right)$ uncountably chromatic?

Theorem 8.5 (65]). In some models of set theory $\mathrm{Chr}\left(\mathrm{EH}\left(\omega_{2}\right)\right)$ can be large. In particular, it is possible to have $\operatorname{Chr}\left(\mathrm{EH}\left(\omega_{2}\right)\right)=2^{\aleph_{2}}$.

We also have the following result which shows that forcing axioms also have some influence on the chromatic number of $\mathrm{EH}\left(\omega_{2}\right)$.

Theorem 8.6 ([11]). PFA imlies that $\operatorname{Chr}\left(\operatorname{EH}\left(\omega_{2}\right)\right) \geq \aleph_{2}$.

Sketch of Proof: Using PFA we shall construct a coloring $c:\left[\omega_{2}\right]^{2} \rightarrow 2$ such that:

(1) There is no $A \subseteq \omega_{2}$ of cardinality $\aleph_{2}$ such that $c^{\prime \prime}[A]^{2}=\{0\}$.

(2) For every $\alpha<\beta<\omega_{2}$, the set $\{\xi<\alpha: c(\xi, \alpha)=c(\xi, \beta)=1\}$ is finite.

Fix an enumeration $X_{\xi}\left(\xi<\omega_{2}\right)$ of all bounded subsets of $\omega_{2}$. Recursively on $\alpha<\omega_{2}$, we shall choose sets $A_{\alpha} \subseteq \alpha$ such that

(3) $A_{\alpha} \cap A_{\beta} \in$ Fin whenever $\alpha \neq \beta$,

(4) $A_{\beta} \cap X_{\xi} \neq \emptyset$ for all $\xi<\beta$ with the property that $X_{\xi} \backslash\left(\bigcup_{\alpha \in D} A_{\alpha}\right)$ is uncountable for all countable $D \subseteq \beta$.

If for some $\beta<\omega_{2}$, the sequence $A_{\alpha}(\alpha<\beta)$ is constructed, choose $B_{\beta} \subseteq \beta$ satisfying (4) in place of $A_{\beta}$ and the weaker form of (3) to the effect that $A_{\alpha} \cap B_{\beta}$ is countable when $\alpha<\beta$. Now define the standard ccc poset to thin out $B_{\beta}$ to a subset $A_{\beta}$ satisfying (3) and (4). When finished, set

$$
c(\{\alpha, \beta\})=1 \text { whenever } \alpha \in A_{\beta} .
$$

It is clear that $c$ has the desired properties. Consider the graph

$$
\mathcal{G}=\left(\omega_{2}, c^{-1}(1)\right) .
$$

Then $\mathcal{G}$ has no independent set of size $\aleph_{2}$, so in particular, its chromatic number is equal to $\aleph_{2}$. On the other hand, the property (2) of the coloring $c$ translates into the fact that the poset $\mathcal{P}$ of all finite $\mathcal{G}$-independent subsets of $\omega_{2}$ satisfies the countable chain condition. So applying $\mathrm{MA}_{\aleph_{1}}$ we conclude that

$$
\operatorname{Chr}\left(\mathcal{G}\lceil\alpha) \leq \aleph_{0} \text { for all } \alpha<\omega_{2}\right. \text {. }
$$

By Theorem 8.2 we conclude that $\mathcal{G} \leq \mathrm{EH}\left(\omega_{2}\right)$, as required.

Problem 8.7. Does PFA imply that $\operatorname{Chr}\left(\operatorname{EH}\left(\omega_{2}\right)\right)>\aleph_{2}$ ?

Let us now give some upper bounds on $\operatorname{Chr}(\operatorname{EH}(\theta))$. For this we need the following standard notion.

Definition 8.8 ([51]). A poset $\mathcal{P}$ is said to satisfy $\sigma$-finite chain condition if it admits a countable decomposition

$$
\mathcal{P}=\bigcup_{n<\omega} \mathcal{P}_{n}
$$

with the property that no $\mathcal{P}_{n}$ contains an infinite set of pairwise incompatible elements. 
Proposition 8.9. If $\theta$ supports a proper uniform $\sigma$-complete ideal $\mathcal{I}$ with the property that the corresponding quotient algebra $\mathcal{P}(\theta) / \mathcal{I}$ satisfies the $\sigma$-finite chain condition, then $\operatorname{Chr}(\mathrm{EH}(\theta)) \leq \aleph_{0}$.

Corollary 8.10. $\operatorname{Chr}(\mathrm{EH}(\theta)) \leq \aleph_{0}$ for every real-valued measurable cardinal $\theta$.

Proposition 8.11. If $\mathcal{U}$ is a uniform ultrafilter on $\theta$, then

$$
\operatorname{Chr}(\operatorname{EH}(\theta)) \leq\left|\omega^{\theta} / \mathcal{U}\right| .
$$

The following result shows that this hypothesis can sometimes be achieved with the minimal possible value for the ultrapower.

Theorem $8.12([38])$. It is consistent relative to the existence of some large cardinals that $\left|\omega^{\omega_{2}} / \mathcal{U}\right|=\aleph_{1}$ for some uniform ultrafilter $\mathcal{U}$ on $\omega_{2}$.

Corollary 8.13 ([38). It is consistent that $\mathrm{Chr}\left(\mathrm{EH}\left(\omega_{2}\right)\right) \leq \aleph_{1}$.

Proposition 8.14. Shift $\left(\mathrm{EH}\left(\mathfrak{c}^{\theta}\right)\right) \leq \mathrm{EH}\left(\omega^{\theta}\right)=\mathrm{EH}(\theta)$.

Proof. Let $\left\{q_{n}: n<\omega\right\}$ be an enumeration of the rationals. Define $\Delta: \mathbb{R}^{2} \rightarrow \omega$ by setting

$$
\Delta(x, y)= \begin{cases}0 & \text { if } x=y \\ n & \text { with } n \text { minimal such that } q_{n} \in(x, y) \text { or } q_{n} \in(y, x) .\end{cases}
$$

Define

by letting

$$
\Phi:\left\{(f, g) \in\left(\mathbb{R}^{\theta}\right)^{2}: f \perp g \text { and } f<_{\operatorname{lex}} g\right\} \rightarrow \mathbb{Z}^{\theta}
$$

$$
\Phi(f, g)(\xi)=\Delta(f(\xi), g(\xi)) \cdot \operatorname{sgn}(f(\xi)-g(\xi))
$$

Note that $\Phi(f, g) \perp \Phi\left(f^{\prime}, g^{\prime}\right)$ whenever $f<_{\operatorname{lex}} g=f^{\prime}<_{\operatorname{lex}} g^{\prime}$ and $f \perp g$ and $f^{\prime} \perp g^{\prime}$. It follows that $\Phi$ is a homomorphism from $\operatorname{Shift}\left(\mathrm{EH}\left(\mathbb{R}^{\theta}\right)\right)$ into $\mathrm{EH}(\theta)$, as required.

Corollary 8.15. $\operatorname{Chr}\left(\mathrm{EH}\left(\mathfrak{c}^{\theta}\right)\right)>\mathfrak{c}$ implies $\operatorname{Chr}(\mathrm{EH}(\theta))>\aleph_{0}$

Proof. This follows from Proposition 8.14 and Theorem 2.4

Theorem $8.16([119])$. $\operatorname{Chr}\left(\mathrm{EH}\left(\omega_{2}\right)\right)>\aleph_{0}$.

Proof. Note that $\mathcal{K}_{\mathfrak{c}^{+}} \leq \mathrm{EH}\left(\mathfrak{c}^{\omega_{2}}\right)$ when $\mathfrak{c} \leq \aleph_{2}$. So by Corollary 8.15, we get the desired conclusion $\operatorname{Chr}\left(\operatorname{EH}\left(\omega_{2}\right)\right)>\aleph_{0}$ in case $\mathfrak{c} \leq \aleph_{2}$. So it remains to reach the same conclusion when $\mathfrak{c}>\aleph_{2}$. Fix

$$
e:\left[\omega_{2}\right]^{2} \rightarrow \omega_{1}
$$

such that $e(\alpha, \gamma) \neq e(\beta, \gamma)$ whenever $\alpha<\beta<\gamma<\omega_{2}$.

Recall the following two properties of the family $\mathcal{F}_{e}$ of intervals of the linearly ordered set $\mathcal{L}_{e}$ constructed above:

(1) $\mathcal{F}_{e}=\bigcup_{\xi<\omega_{2}} \mathcal{F}_{e}^{\xi}$ such that $\mathcal{F}_{e}^{\xi} \subseteq \mathcal{F}_{e}^{\eta}$ whenever $\xi<\eta$, and such that for every $\xi<\omega_{2}$ the family $\mathcal{F}_{e}^{\xi}$ can be decomposed into countably many families consisting of pairwise disjoint intervals.

(2) Since $\mathbb{R} \nsubseteq L[e]$, the family $\mathcal{F}_{e}$ itself cannot be decomposed into countably many subfamilies consisting of pairwise disjoint intervals. 
Let $\left(\mathcal{F}_{e}\right.$, Int) be the intersection graph of $\mathcal{F}_{e}:(I, J) \in$ Int if and only if $I \cap J \neq \emptyset$. For every $\xi<\omega_{2}$, we fix a map $g_{\xi}: \mathcal{F}_{e}^{\xi} \rightarrow \omega$ such that $I \cap J \neq \emptyset \Rightarrow g_{\xi}(I) \neq g_{\xi}(J)$. Define $\Phi: \mathcal{F}_{e} \rightarrow \omega^{\omega_{2}}$ by setting

$$
\Phi(I)(\xi)= \begin{cases}1 & \text { if } I \notin \mathcal{F}_{e}^{\xi} \\ g_{\xi}(I) & \text { if } I \in \mathcal{F}_{e}^{\xi}\end{cases}
$$

Claim 1. $\Phi:\left(\mathcal{F}_{e}, \operatorname{Int}\right) \rightarrow\left(\mathrm{EH}\left(\omega_{2}\right), \perp\right)$ is a homomorphism, or in other words, $I \cap J \neq \emptyset$ implies $\Phi(I) \perp \Phi(J)$.

It follows that $\operatorname{Chr}\left(\operatorname{EH}\left(\omega_{2}\right)\right) \geq \operatorname{Chr}\left(\mathcal{F}_{e}\right.$, Int). Since by $(2), \operatorname{Chr}\left(\mathcal{F}_{e}, \operatorname{Int}\right)>\aleph_{0}$, the proof of Theorem 8.16 is finished.

Problem 8.17. Investigate the chromatic number of $\operatorname{EH}(\theta)=\left(\omega^{\theta}, \perp\right)$ for other values of $\theta$ such as for example $\theta=\omega_{3}, \omega_{4}, \omega_{5}, \ldots$, or $\theta=\omega_{\omega+1}$.

Theorem 8.18 ([116]). For every infinite cardinal $\theta$, the inequality $\operatorname{Chr}(\mathrm{EH}(\theta)) \leq$ $\aleph_{0}$ implies $\theta \rightarrow\left(\omega_{1}\right)_{\omega_{1}, \omega}^{<\omega} \cdot{ }^{17}$ In particular, it there is an infinite cardinal $\theta$ such that $\operatorname{Chr}(\operatorname{EH}(\theta)) \leq \aleph_{0}$ then $0^{\sharp}$ exists.

Sketch of Proof: The proof will follow closely the proof of Theorem 7.1 above. For $A \in\left[\omega_{1} \cup \omega_{1}^{\theta}\right]^{\aleph_{0}}$, set

$$
D_{A}=\{\alpha<\theta:(\forall f \in A) f(\alpha) \in A\} .
$$

Let

$$
\mathcal{S}_{\theta}=\left\{A \in\left[\omega_{1} \cup \omega_{1}^{\theta}\right]^{\aleph_{0}}: A \cap \omega_{1} \in \omega_{1} \text { and } \sup \left(D_{A}\right)<\theta\right\} .
$$

As before, it suffices to show that $\operatorname{Chr}(\operatorname{EH}(\theta)) \leq \aleph_{0}$ implies that $\mathcal{S}_{\theta}$ is not stationary. (Note that our assumption $\operatorname{Chr}(\operatorname{EH}(\theta)) \leq \aleph_{0}$ implies that in particular $\left.\operatorname{cf}(\theta)>\omega_{1}.\right)$

Let $\mathcal{T}$ be the collection of all sequences $t=\left(t_{\xi}: \xi \leq \ell(t)\right)$ such that:

(a) $\ell(t)<\omega_{1}$ and $t_{\xi} \in \mathcal{S}_{\theta}$ for all $\xi \leq \ell(t)$,

(b) $t_{\xi} \subseteq t_{\eta}$ and $t_{\xi} \cap \omega_{1}<t_{\eta} \cap \omega_{1}$ for all $\xi<\eta \leq \ell(t)$,

(c) $t_{\lambda}=\bigcup_{\xi<\lambda} t_{\xi}$ for all limit $\lambda \leq \ell(t)$.

We consider $\mathcal{T}$ as a tree ordered by end-extension. For $\delta<\theta$, let

$$
\mathcal{T}^{\delta}=\left\{t \in \mathcal{T}:(\forall \xi \leq \ell(t)) D_{t_{\xi}} \subseteq \delta\right\} .
$$

Claim 1. For every $\delta<\theta$, the subtree $\mathcal{T}^{\delta}$ can be covered by countably many antichains.

Proof. Fix $\delta<\theta$. By the Pressing Down Lemma for trees, it suffices to define a regressive mapping

$$
H: \mathcal{T}^{\delta} \rightarrow \mathcal{T}^{\delta}
$$

such that every of its pre-images $H^{-1}(s) s \in \mathcal{T}^{\delta}$ allows a decomposition into countably many antichains. For $t$ a successor node of $\mathcal{T}^{\delta}$, let $H(t)$ be its immediate predecessor. Consider now $t \in \mathcal{T}^{\delta}$ of limit height $\ell(t)$. Since $\delta \notin D_{t_{\xi}}$ for $\xi=\ell(T)$ there is a function

$$
f_{t} \in t_{\ell(t)} \cap \omega_{1}^{\theta} \text { such that } f_{t}(\delta) \geq t_{\ell(t)} \cap \omega_{1} .
$$

Let $H(t)$ be the minimal initial segment $s$ of $t$ such that $f_{t} \in s_{\ell(s)}$.

\footnotetext{
${ }^{17}$ Recall that $\theta \rightarrow\left(\omega_{1}\right)_{\omega_{1}, \omega}^{<\omega}$ means that for every mapping $f: \theta^{<\omega} \rightarrow \omega_{1}$ there exists uncountable $X \subseteq \theta$ such that $\left|f^{\prime \prime} X^{<\omega}\right| \leq \aleph_{0}$.
} 
We need to show that for every $s \in \mathcal{T}^{\delta}$, the pre-image $H^{-1}(s)$ can be decomposed into countably many antichains. Fix an $s \in \mathcal{T}^{\delta}$. For $f \in t_{\ell(s)} \cap \omega_{1}^{\theta}$, set

$$
W_{f}=\left\{t \in H^{-1}(s): f_{t}=f\right\} .
$$

Since $H^{-1}(s)=\bigcup_{f \in s_{\ell(s)}} W_{f}$ is a countable union, it suffices to show that each set $W_{f}$ can be covered by countably many antichains. To see this first observe that

$$
f(\delta) \geq t_{\ell(t)} \cap \omega_{1} \text { for all } t \in W_{f} .
$$

According to the condition (b) from the definition of $\mathcal{T}^{\delta}$, this in particular means that $W_{f}$ contains no chain of order type $>f(\delta)$. So $W_{f}$ as a subtree of $\mathcal{T}^{\delta}$ has only countably many levels, so in particular can be covered by countably many antichains.

For $\delta<\theta$, fix $G_{\delta}: \mathcal{T}^{\delta} \rightarrow \omega$ such that $G_{\delta}^{-1}(n)$ is an antichain for all $n<\omega$. Define $\Phi: \mathcal{T} \rightarrow \omega^{\theta}$, by

$$
\Phi(t)(\delta)= \begin{cases}0 & \text { if } t \notin \mathcal{T}^{\delta} \\ G_{\delta}(t) & \text { if } t \in \mathcal{T}^{\delta}\end{cases}
$$

Then $\Phi$ is a homomorphism from the comparability graph of $\mathcal{T}$ into $\operatorname{EH}(\theta)$. It follows, in particular, that the comparability graph of $\mathcal{T}$ is countably chromatic, or equivalently, that the whole tree $\mathcal{T}$ can be decomposed into countably many antichains. So the proof is finished, once we show the following.

Claim 2. If $\mathcal{S}_{\theta}$ is stationary, the corresponding tree $\mathcal{T}$ cannot be decomposed into countably many antichains.

Proof. Consider a decomposition $\mathcal{T}=\bigcup_{n<\omega} \mathcal{X}_{n}$. Assuming that $\mathcal{S}_{\theta}$ is stationary we will show that one of the $\mathcal{X}_{n}$ 's is not an antichain. Choose a countable elementary submodel $M$ of $H_{\left(2^{\theta}\right)^{+}}$containing all these objects such that $M \cap\left(\omega_{1} \cup \omega_{1}^{\theta}\right) \in \mathcal{S}_{\theta}$. Then it is possible to build an increasing sequence $\left\{t_{n}: n<\omega\right\}$ in $\mathcal{T} \cap M$ with an upper bound $t \in \mathcal{T}$ such that:

(1) $t_{\ell(t)}=M \cap\left(\omega_{1} \cup \omega_{1}^{\theta}\right)$,

(2) for every $n<\omega$, either $t_{n} \in \mathcal{X}_{n}$ or else no extension of $t_{n}$ belongs to $\mathcal{X}_{n}$.

Pick an $n<\omega$ such that $t \in \mathcal{X}_{n}$. Then $t_{n}$ must also belong to $\mathcal{X}_{n}$. So, $\mathcal{X}_{n}$ contains to distinct comparable nodes $t$ and $t_{n}$.

Corollary 8.19. In Gödel's constructible universe $L, \operatorname{Chr}(\mathrm{EH}(\theta))>\aleph_{0}$ for all infinite cardinals $\theta$.

Problem 8.20. Evaluate $\operatorname{Chr}(\operatorname{EH}(\theta))$ in the Gödel constructible universe $L$.

Corollary 8.21. The weak compactness of an infinite cardinal $\theta$ is not sufficient for the conclusion $\mathrm{Chr}(\mathrm{EH}(\theta)) \leq \aleph_{0}{ }^{18}$

\section{The Countable Chain Condition on Graphs and Hypergraphs}

Recall that a hypergraph is a structure of the form $\mathcal{H}=(X, E)$ where $X$ is a set and $E$ a collection of nonempty finite subsets of $X$. When every element of $E$ has some fixed size $n$, we call $\mathcal{H}=(X, E)$ an $n$-hypergraph. Thus a graph is nothing more than a 2-hypergraph. Elements of $E$ are called hyperedges. A subset $D$ of $X$ is $\mathcal{H}$-discrete if it includes no hyperedge.

\footnotetext{
${ }^{18}$ Recall that measurability of $\theta$ is sufficient for this conclusion.
} 
Definition 9.1. The chromatic number, $\operatorname{Chr}(\mathcal{H})$, of a hypergraph is the minimal cardinality of a partition of $X$ into $\mathcal{H}$-discrete subsets of $X$. Note that this agrees with the notion of chromatic number for graphs.

It is well known that forbidding complete subgraphs (or even $\mathcal{K}_{3}$ ) is not a sufficient condition for an upper bound on the chromatic number of a given graph. It turns out however that the following natural strengthening of the condition $\mathcal{K}_{\aleph_{1}} \leq \mathcal{H}$ has some facility for giving us such an upper bound.

Definition 9.2. A hypergraph $\mathcal{H}=(X, E)$ satisfies the countable chain condition if for every uncountable family $\mathcal{F}$ of finite $\mathcal{H}$-discrete subsets of $X$ there exist $p \neq q$ in $\mathcal{F}$ whose union $p \cup q$ is also $\mathcal{H}$-discrete.

So in this context the following statement falls naturally into the category of graphtheoretic dichotomy results of our interest here.

Definition 9.3 ([112]). [The statement $\Sigma]$ For every hypergraph $\mathcal{H}=(X, E)$ on a set $X$ of cardinality at most continuum, either $\mathcal{H}$ fails to satisfy the countable chain condition, or else $\operatorname{Chr}(\mathcal{H}) \leq \aleph_{1}$. If we restrict $\Sigma$ to $n$-hypergraphs, the corresponding weaker (though in some sense more interesting) statement is denoted by $\Sigma^{n}$. The restrictions to the corresponding classes of Borel hypegraph ${ }^{19}$ are denoted by $\Sigma_{B}$ or $\Sigma_{B}^{n}$, respectively.

Question 9.4. Is any of the statements $\Sigma^{2}, \Sigma^{3}, \cdots, \Sigma$ equivalent to $\mathrm{CH}$ ?

It has been proved in [112] that $\Sigma_{B}$ is consistent with the continuum large so there is no way to capture the full strength of $\mathrm{CH}$ by using just Borel structures 20 . However the following sample results show that $\Sigma$ and $\Sigma_{B}$ do capture basically all the standard consequences of $\mathrm{CH}$.

Theorem 9.5 ([112]). $\Sigma$ implies that every function $f: \omega_{2} \times \omega_{2} \rightarrow \omega$ must be constant on some product of two infinite subsets of $\omega_{2}$.

Theorem 9.6 ([112]). $\Sigma$ implies that if some inner model satisfies $\mathrm{MA}_{\omega_{2}}$ then $0^{\sharp}$ exists.

Theorem 9.7 ([112]). $\Sigma$ implies that every free ultrafilter $\mathcal{U}$ on $\mathbb{N}$ has $\pi$-character $\aleph_{1}$, i.e., there is a family $\mathcal{F}$ of $\aleph_{1}$ subsets of $\mathbb{N}$ such that every element of $\mathcal{U}$ includes one of $\mathcal{F}$.

Sketch of Proof. Apply $\Sigma$ to the hypergraph $\mathcal{H}=(\mathcal{U}, \mathcal{E})$, where $\mathcal{E}$ is the collection of all finite subsets $\mathcal{X}$ of $\mathcal{U}$ for which we can find an integer $k$ such that

$$
|(\bigcap \mathcal{X}) \cap\{0,1, \ldots, k-1\}|<|\{\Delta(A, B): A, B \in \mathcal{X}, A \neq B\}| .
$$

Note that while the vertex-set $\mathcal{U}$ of this hypergraph is not Borel its hyperedge relation is. So this application of $\Sigma$ allows large continuum.

Theorem 9.8 ([112]). $\Sigma_{B}$ implies each of the following standard consequences of the Continuum Hypothesis:

\footnotetext{
${ }^{19}$ A hypergraph $\mathcal{H}=(X, E)$ is Borel if $X$ is a Polish space and $E$ is a Borel collection of finite subsets of $X$

${ }^{20}$ It is shown in 112 that large continuum is consistent with $\Sigma$ applied to any hypergraph $\mathcal{H}=(X, E)$ whith $X$ a set of reals and $E=E^{*} \cap[X]^{<\omega}$ where $E^{*}$ is a Borel set of finite sets of reals. Thus, the vertex set $X$ need not be Borel.
} 
(a) There is a family $\mathcal{F}$ of $\aleph_{1}$ nowhere dense sets of reals such that every nowhere dense set of reals is included in a member from $\mathcal{F}$.

(b) There is a family $\mathcal{F}$ of $\aleph_{1}$ measure zero sets of reals such that every measure zero set of reals is included in a member from $\mathcal{F}$.

Sketch of Proof. Since the ideal of meager subsets of $\mathbb{R}$ is Tukey-reducible to the ideal of measure zero subsets of $\mathbb{R}$ (see $[42$ and Section 24.1 below) the statement (a) is a consequence of the statement (b). To see that $\Sigma_{B}$ implies (b), consider the hypergraph $\mathcal{H}=(\mathcal{A}, \mathcal{E})$, where $\mathcal{A}$ is the collection of all compact subsets of $\mathbb{R}$ of measure $>1 / 2$ and where $\mathcal{E}$ is the family of all finite subsets $\mathcal{K}$ of $\mathcal{A}$ such that $\bigcap \mathcal{K}$ has measure $\leq 1 / 2$.

The more interesting finite-dimensional $\Sigma^{n}$ have similar though weaker consequences.

Theorem 9.9 ([112]). $\Sigma^{3}$ implies $\operatorname{cf}(\mathfrak{c})=\aleph_{1}$.

Sketch of Proof. To a given function $f: \mathbb{N}^{\mathbb{N}} \rightarrow \mathbb{N}^{\mathbb{N}}$ we associate the triple system $\mathcal{H}=\left(\mathbb{N}^{\mathbb{N}}, E_{f}\right)$ by letting $\{a, b, c\} \in E_{f}$ if and only if either $f \nmid\{a, b, c\}$ is not one-to-one, or

$$
|\{\Delta(a, b), \Delta(a, c), \Delta(b, c)\}|>\left.|\{\Delta(f(a), f(b)), \Delta(f(a), f(c)), \Delta(f(b), f(c))\}|\right|^{21}
$$

Then $\mathcal{H}_{f}$ satisfies the countable chain condition and the function $f$ is continuous on any subset $X$ of $\mathbb{N}^{\mathbb{N}}$ which includes no triple from $E_{f}$. Now apply $\Sigma^{2}$ to the triple system $\mathcal{H}_{f}$ associated to a one-to-one function $f: \mathbb{N}^{\mathbb{N}} \rightarrow \mathbb{N}^{\mathbb{N}}$ that is not continuous on any set of size continuum.

Theorem $9.10([112]) . \Sigma_{B}^{3}$ implies $\mathfrak{d}=\aleph_{1}$.

Sketch of Proof. Apply $\Sigma_{B}^{3}$ to the Borel triple system $\mathcal{H}=\left(\mathbb{N}^{\mathbb{N}}, E_{d}\right)$ where $E_{d}$ is the collection of all triples $\{a, b, c\} \subseteq \mathbb{N}^{\mathbb{N}}$ such that $|\{\Delta(a, b), \Delta(a, c), \Delta(b, c)\}|=1$.

Theorem $9.11([112])$. $\Sigma^{2}$ implies $\mathfrak{b}=\aleph_{1}$.

Sketch of Proof. Choose a subset $X$ of $\mathbb{N}^{\mathbb{N}}$ consisting of increasing functions from $\mathbb{N}$ in $\mathbb{N}$ such that $X$ is well-ordered and unbounded relative to the ordering of eventual dominance of $\mathbb{N}^{\mathbb{N}}$. Apply $\Sigma^{2}$ to the graph $\mathcal{H}=\left(X, E_{b}\right)$, where $E_{b}$ is the collection of all 2-element subsets of $X$ such that either $(\forall n) a(n) \leq b(n)$ or else $(\forall n) b(n) \leq a(n) 2^{22}$

\section{Part 2. Topologically Presented Graphs}

In this part we look at compactness of chromatic numbers of topologically presented graphs, again with the aim of finding classes with small compactness numbers and interesting consequences. Thus, we shall be interested in graphs of the form

$$
\mathcal{G}=(X, E),
$$

where $X$ is a topological space and $E$ a symmetric irreflexive subset of $X^{2}$ with some prescribed topological complexity such as, for example, open, closed, $\mathrm{F}_{\sigma}$, etc. We have already seen above that spaces $X$ with large discrete subspaces will support

\footnotetext{
${ }^{21}$ Note that no matter what the function $f$ is, the triple system $\mathcal{H}_{f}$ can be represented in the form $(X, E)$ where $X$ is some set of reals and where $E$ is the restriction to $X$ of some Borel triple system on $\mathbb{R}$. So this application of $\Sigma^{3}$ also alows the continuum to be large.

${ }^{22}$ Note that while $X$ is not Borel, the relation $E_{b}$ is Borel. So the form of $\Sigma_{2}$ used here also allows the continuum to be large.
} 
graphs of small complexity but large compactness number. In fact, at the current stage of our knowledge, we can say something only when $X$ is a separable metric space. So from now on, the vertex set $X$ of a topologically given graph $\mathcal{G}=(X, E)$ will be assumed to be at least separable metric. The following well known fact ${ }^{23}$ shows that if we wish to keep the vertex set to be an arbitrary separable metric space we must add severe restrictions on the complexity of the edge-relation.

Proposition $9.12(40 ; 21 \mathrm{~F})$. For every symmetric irreflexive relation $E \subseteq \mathfrak{p}^{2}$ there is a sequence $\left\{a_{\xi}: \xi<\mathfrak{p}\right\} \subseteq[\omega]^{\omega}$ such that $(\xi, \eta) \in E$ iff $a_{\xi} \cap a_{\eta} \in$ Fin.

In other words, an arbitrary graph on a vertex set of cardinality at most $\mathfrak{p}$ is isomorphic to a graph whose vertex set is a separable metric space and whose edge relation if $F_{\sigma}$. Thus, we are restricted to considering the following two compactness numbers.

Definition 9.13. The open graph compactness number, $\kappa_{\mathrm{O}}$, is the minimal number $\kappa$ with the property that every open graph $\mathcal{G}=(X, E)$ on a separable metric space is countably chromatic if all of its subgraphs of cardinality $<\kappa$ are countably chromatic. The closed graph compactness number, $\kappa_{\mathrm{C}}$, is the minimal number $\kappa$ with the property that every closed graph $\mathcal{G}=(X, E)$ on a separable metric space is countably chromatic if all of its subgraphs of cardinality $<\kappa$ are countably chromatic.

Note that we always have the inequalities $\mathfrak{c}^{+} \geq \kappa_{\mathrm{O}}$ and $\mathfrak{c}^{+} \geq \kappa_{\mathrm{C}}$. So in this context the cardinals have their nontrivial realizations only when they are smaller or equal to the continuum.

Problem 9.14. Are $\kappa_{\mathrm{O}}$ and $\kappa_{\mathrm{C}}$ regular cardinals whenever nontrivially realized?

\section{The Open Graph Compactness Number and the Bounding number}

Recall the following important cardinal characteristic of the continuum

$$
\mathfrak{b}=\min \left\{|\mathcal{F}|: \mathcal{F} \subseteq \mathbb{N}^{\mathbb{N}},\left(\forall g \in \mathbb{N}^{\mathbb{N}}\right)(\exists f \in \mathcal{F}) f \nless^{*} g\right\} .
$$

Note that this is a regular cardinal and note the following immediate consequence of the definition of this cardinal.

Proposition 10.1. There is always $a<^{*}$-well-ordered $<^{*}$-unbounded chain of order type $\mathfrak{b}$ in $\left(\mathbb{N}^{\mathbb{N}},<^{*}\right)$.

The purpose of this section is to prove the following.

Theorem $10.2([108]) \cdot \mathfrak{b} \leq \kappa_{\mathrm{O}}$ if $\kappa_{\mathrm{O}}$ is a regular cardinal and $\mathfrak{b} \leq \kappa_{\mathrm{O}}^{+}$if $\kappa_{\mathrm{O}}$ is a singular cardinal.

Proof. Assume the contrary, that $\mathfrak{b}>\theta$, where $\theta$ be the minimal regular cardinal $\geq \kappa_{\mathrm{O}}$. By Proposition 10.1, we can choose a $<^{*}$-increasing $<^{*}$-unbounded sequence $A=\left\{a_{\xi}: \xi<\theta\right\}$ in $\left(\mathbb{N}^{\mathbb{N}},<^{*}\right)$ consisting of strictly increasing mappings from $\mathbb{N}$ into $\mathbb{N}$. Since $A$ is bounded in $\left(\mathbb{N}^{\mathbb{N}},<^{*}\right)$ we can choose a maximal $<^{*}$-chain $B$ consisting of upper bounds of $A$. Note that if $b$ is an upper bound of $A$ then so is $b-1^{24}$ and so by its maximality the set $B$ has no $<^{*}$-minimal element. Therefore, by our

\footnotetext{
${ }^{23}$ where $\mathfrak{p}$ is the minimal cardinality of a family $\mathcal{F} \subseteq[\omega]^{\omega}$ that is closed under finite intersection such that there is no infinite $b \subseteq \mathbb{N}$ such that $b \backslash a \in$ Fin for all $a \in \mathcal{F}$.

${ }^{24}$ defined by $(b-1)(n)=\max (0, b(n)-1)$.
} 
assumption $\mathfrak{b}>|A|$, the chain $\left(B,<^{*}\right)$ must have uncountable coinitiality. Let $B^{*}$ be the set of all finite changes of elements of $B$, and let

$$
X=\left\{(a, b) \in A \times B^{*}:(\forall n) a(n) \leq b(n)\right\} .
$$

Then $X$ is a separable metric space with the topology induced from $\mathbb{N}^{\mathbb{N}} \times \mathbb{N}^{\mathbb{N}}$. Consider the following open edge relation on $X$,

$$
E=\left\{\left((a, b),\left(a^{\prime}, b^{\prime}\right)\right) \in X^{2}:(\exists n) a(n)>b^{\prime}(n) \text { or }(\exists n) a^{\prime}(n)>b(n)\right\} .
$$

This gives us the open graph $\mathcal{G}=(X, E)$ that will be subject to the definition of $\kappa_{\mathrm{O}}$ once we establish the following.

Claim 1. Every subgraph $\mathcal{G}\left\lceil Y\right.$ of cardinality $<\kappa_{\mathrm{O}}$ is countably chromatic.

Proof. Choose $c$ in $A$ such that $a<^{*} c$ for every $a$ appearing as a first coordinate of an element of $Y$. Let $\left\{c_{k}: k<\omega\right\}$ lists all finite changes of $c$. For $k<\omega$, let

$$
Y_{k}=\left\{(a, b) \in Y:(\forall n) a(n) \leq c_{k}(n) \leq b(n)\right\}
$$

Then $Y=\bigcup_{k<\omega} Y_{k}$ is a coloring witnessing $\operatorname{Chr}\left(\mathcal{G}\lceil Y) \leq \aleph_{0}\right.$.

By the definition of $\kappa_{\mathrm{O}}$, it follows that $\operatorname{Chr}(\mathcal{G}) \leq \aleph_{0}$. So let $X=\bigcup_{k<\omega} X_{k}$ be a good coloring witnessing this. Since $B$ has uncountable coinitiality, there must be $k$ such that the first projection $A_{k}$ of $X_{k}$ is cofinal in $\left(A,<^{*}\right)$ while the second projection $B_{k}$ of $X_{k}$ is coinitial in $\left(B^{*},<^{*}\right)$. Define $c \in \mathbb{N}^{\mathbb{N}}$, by

$$
c(n)=\min \left\{b(n): b \in B_{k}\right\} .
$$

Then $A<^{*} c<^{*} B$, and so $\{c\} \cup B$ contradicts the maximality of $B$. This completes the proof

\section{A Conjecture about open graphs on SEPARAble metric SPACES}

The purpose of this section is to expose the following conjecture that is quite analogous to Galvin's and Rado's conjectures considered above in Part I.

Definition 11.1 ([108]). [Todorcevic's Conjectur $\AA^{25} \kappa_{\mathrm{O}}=\aleph_{2} \cdot{ }^{26}$

Clearly, $\mathrm{CH}$ implies TC for the trivial reason that in this case we have the equality $\kappa_{\mathrm{O}}=\mathfrak{c}^{+}$. So since we are interested in the nontrivial realizations of $\kappa_{\mathrm{O}}$ we have to look for appearances of $\mathrm{TC}$ in the context of the negation of $\mathrm{CH}$. The following result establishes one such case.

Theorem 11.2 ([108]). The Proper Forcing Axiom implies that $\kappa_{\mathrm{O}}=\aleph_{2}$.

Remark 11.3. More precisely, we shall show that given an open graph $\mathcal{G}=(X, R)$ on a separable metric space $X$ such that $\operatorname{Chr}(\mathcal{G})>\aleph_{0}$ there is a proper poset which forces $\mathcal{K}_{\aleph_{1}} \leq \mathcal{G}$.

[Sketch of Proof of Theorem 11.2 Let $\mathcal{P}$ be the collection of all pairs $p=\left\langle H_{p}, \mathcal{N}_{p}\right\rangle$ with the following properties:

\footnotetext{
${ }^{25}$ The Todorcevic Conjecture, TC in short, shows up implicitly first in 108 as the main part of an axiom about open graphs introduced there (see also Definition 11.6 below). The name was suggested by the Editor of this Bulletin.

${ }^{26}$ In other words, an open graph on a separable metric space is countably chromatic if and only if all of its subgraphs of cardinality at most $\aleph_{1}$ are countably chromatic.
} 
(1) $H_{p}$ is a finite clique of $\mathcal{G}$, i.e., $H$ is a finite subset of $X$ such that for every $x \neq y \in H_{p}$ the pair $\{x, y\}$ is an edge of $\mathcal{G}$,

(2) $\mathcal{N}_{p}$ is a finite $\in$-chain of countable elementary submodels $N$ of $\left(H_{\mathfrak{c}^{+}}, \in\right)$,

(3) $\mathcal{N}_{p}$ separates $H_{p}$, that is,

$$
\left(\forall x \neq y \in H_{p}\right)\left(\exists N \in \mathcal{N}_{p}\right)|N \cap\{x, y\}|=1,
$$

(4) $\left(\forall N \in \mathcal{N}_{p}\right)\left(\forall x \in H_{p} \backslash N\right)(\forall Y \in N)\left[x \in Y \rightarrow Y^{2} \cap R \neq \emptyset\right]$.

and we set $p \leq q$ if and only if

(5) $H_{p} \supseteq H_{q}$ and $\mathcal{N}_{p} \supseteq \mathcal{N}_{q}$.

Theorem 11.2 follows easily from the following Claim.

Claim 1. $\mathcal{P}$ is proper.

[Sketch of the proof of Claim 1] Let $p \in M \prec\left(H_{\left(2^{\mathfrak{c}}\right)^{+}}, \in\right)$. We prove that

$$
q=\left(H_{p}, \mathcal{N}_{p} \cup\left\{M \cap H_{\mathfrak{c}^{+}}\right\}\right)
$$

is an $(M, \mathcal{P})$-generic condition. That is, we need to show

$(\forall \mathcal{D} \in M$ such that $\mathcal{D} \subseteq \mathcal{P}$ is dense open $)(\forall r \leq q)(\exists \bar{r} \in \mathcal{D} \cap M) \bar{r} \sim r{ }^{27}$

We may assume $r \in \mathcal{D}$. Let $n=\left|H_{r} \backslash M\right|$. Let

$$
x^{r}=\left\langle x_{0}^{r}, x_{1}^{r}, \ldots, x_{n-1}^{r}\right\rangle,
$$

where

$$
H_{r} \backslash M=\left\{x_{0}^{r}, x_{1}^{r}, \ldots, x_{n-1}^{r}\right\},
$$

with indexing chosen according to the $\in$-ordering of $\mathcal{N}_{r}$ :

$$
(\forall i<n-1)\left(\exists N \in \mathcal{N}_{r}\right)\left[x_{i} \in N \quad \& \quad x_{i+1} \notin N\right] .
$$

Let

$$
\mathcal{F}=\left\{x \in X^{n}: \exists s \leq r \cap M\left[s \in \mathcal{D} \quad \& \quad x^{s}=x\right]\right\} .
$$

Then $\mathcal{F} \in M$ and the family $\mathcal{F}$ is large in the following precise sense.

Subclaim 1.1. The family $\mathcal{F}$ of $n$-tuples is Namba relative to the co-ideal

$$
Q=\left\{Y \subseteq X: \operatorname{Chr}\left(\mathcal{G}\lceil Y)>\aleph_{0}\right\},\right.
$$

or in other words the following formula is true

$$
Q x_{0} Q x_{1} \ldots Q x_{n-1}\left\langle x_{0}, x_{1}, \ldots, x_{n-1}\right\rangle \in \mathcal{F}{ }^{28}
$$

Note that Namba families of $n$-tuples of elements of $X$ form a nontrivial $\sigma$-complete coideal of subsets of $X^{n}$.

Subclaim 1.2. For almost all $x \in \mathcal{F}$ in the sense of Namba, there is $y \in \mathcal{F}$ such that

$$
\forall i<n\left[\left(x_{i}, y_{i}\right) \in R\right]
$$

\footnotetext{
${ }^{27}$ Here, $\sim$ denotes the compatibility relation of $\mathcal{P}$.

${ }^{28}$ Recall that for a formula $\varphi(v)$ with one free variable $v$ ranging over the set $X$, the formula $Q x \varphi(v)$ is interpreted as saying that $\{x \in X: \varphi(x)\} \in Q$.
} 
Proof. Induction on $n$. Let

$$
\mathcal{H}_{0}=\left\{x \in X^{n-1}: \operatorname{Chr}\left(\mathcal{G}\left\lceil Y_{x}\right)>\aleph_{0} \text { for } Y_{x}=\{a \in X: x \frown a\} \in \mathcal{F}\right\} .\right.
$$

Then from the assumption that $\mathcal{F}$ is a Namba subset of $X^{n}$, we conclude that $\mathcal{H}_{0}$ is a Namba subset of $X^{n-1}$. Fix a countable base $\mathcal{B} \in M$ for $X$. Since for $x \in \mathcal{H}_{0}$ we have that in particular $Y_{x}^{2} \cap R \neq \emptyset$, and since $R$ is open, we can fix $U_{x}, V_{x} \in \mathcal{B}$ such that

(1) $U_{x} \cap V_{x}=\emptyset$,

(2) $U_{x} \cap Y_{x} \neq \emptyset \neq V_{x} \cap Y_{x}$,

(3) $U_{x} \times V_{x} \subseteq R$.

Find Namba $\mathcal{H}_{1} \subseteq \mathcal{H}_{0}$ and $U, V \in \mathcal{B}$ such that

$$
\left(\forall x \in \mathcal{H}_{1}\right)\left[U_{x}=U \quad \& \quad V_{x}=V\right] .
$$

By the inductive hypothesis there exist $x, y \in \mathcal{H}_{1}$ such that

$$
(\forall i<n-1)\left(x_{i}, y_{i}\right) \in R \text {. }
$$

Find $a \in U$ such that $x \frown a \in \mathcal{F}_{0}$. Find $b \in V$ such that $y \frown b \in \mathcal{F}_{0}$. Then $x \frown a$ and $y \smile b$ are as required.

Note that this finishes the proof of Claim 1 as well as the proof of Theorem 11.2 .

As it will be clear from an example of a closed graph given below (see Example 19.1), we have the following result which shows that the nontrivial realization of the closed graph compactness number is incompatible with PFA.

Theorem 11.4. PFA implies that the closed graph compactness number $\kappa_{\mathrm{C}}$ has only the trivial realization, or more precisely, that $\kappa_{\mathrm{C}}=\mathfrak{c}^{+}$.

It turns out that TC is a compactness principle with strong influence on structures that are related in some way to the set of real numbers. For example, note the following consequence of Theorem 10.2 .

Theorem 11.5 ([108]). TC implies $\mathfrak{b} \leq \aleph_{2}$.

It turns out that $\mathrm{TC}$ does not imply $\mathfrak{c} \leq \aleph_{2}$ since adding any number of Cohen reals to a model of $\mathrm{CH}$ produces a model of $\mathrm{TC}$ while the continuum is arbitrarily large (see [29]).

As remarked above (Remark 11.3, the proof of Theorem 11.2 gives the following stronger form of the principle TC proven to be of considerable and of independent interest.

Definition 11.6 (108). [Todorcevic's Axiom] Let TA denote the statement that for every open graph $\mathcal{G}=(X, E)$ on a separable metric space $X$ either $\operatorname{Chr}(\mathcal{G}) \leq \aleph_{0}$, or $\mathcal{K}_{\aleph_{1}} \leq \mathcal{G}$.

Thus, $\mathrm{TA}$ is a strengthening of $\mathrm{TC}$ in the sense that $\mathrm{TA}=\mathrm{TC}+\mathrm{TA}_{\aleph_{1}}$, where $\mathrm{TA}_{\aleph_{1}}$ denotes the restriction of $\mathrm{TA}$ to separable metric spaces of cardinality at most $\aleph_{1}$. The name was again suggested by the Editor of this Bulletin (see, also, 35]). Originally in [108, we have used the name Open Coloring Axiom for TA but the name has already been used in [1] for a rather different axiom which, in our terminology here, applies to graphs $\mathcal{G}=(X, E)$ on separable metric spaces $X$ of size $\aleph_{1}$ with edge relations $E$ clopen rather than open and asserts that the vertex set $X$ can be covered by countably many sets that are either $\mathcal{G}$-complete or $\mathcal{G}$-discrete. It should also be noted that the paper [1] does have an axiom, denoted 
there by SOCA1 (see 11, p.136), which is in fact stronger than our $\mathrm{TA}_{\aleph_{1}}$ but which unfortunately fails if one does not restrict sets to be of cardinality at most $\aleph_{1}$ (see, for example, Example 19.1 below).

We have already noted that the proof of Theorem 11.2 establishes also the following fact which we now state for the record.

Theorem 11.7 ([108]). The Proper Forcing Axiom implies TA.

\section{The Open Graph Axiom, the Bounding Number, and the Continuum}

Theorem $12.1\left(108{ }^{29}\right)$. TA implies $\mathfrak{b}=\aleph_{2}$.

Question 12.2. Does TA imply $\mathfrak{c}=\aleph_{2}$ ?

We give some details from the proof of Theorem 12.1 as they are of independent interest. There are two ingredients in the proof of the equality $\mathfrak{b}=\aleph_{2}$ from TA:

(1) Gaps in quotient structures $\mathcal{P}(\mathbb{N}) /$ Fin, $\mathbb{N}^{\mathbb{N}} /$ Fin, ...

(2) Oscillation theory of $\mathbb{N}^{\mathbb{N}}$ and of $\mathcal{P}(\mathbb{N})$.

We start by first describing the basic ideas behind the oscillation theory as developed in [107] (see also [108, [111, and [76].) For $x, y \in \mathbb{N}^{\uparrow \leq \mathbb{N}}$ (the set of all nondecreasing finite or infinite sequences of integers), let

$$
D(x, y)=\{n: x(n) \neq y(n)\} .
$$

Let $E(x, y)$ be an equivalence relation on $D(x, y)$ defined by

$$
\begin{gathered}
m E(x, y) n \\
\text { iff } \\
\forall k \in[m, n](x(m)<y(m) \Longleftrightarrow x(k)<y(k) \Longleftrightarrow x(n)<y(n)) .
\end{gathered}
$$

Let

$$
\operatorname{osc}(x, y)=|D(x, y) / E(x, y)|
$$

Theorem 12.3 (107]). Suppose $X$ is a totally ordered and unbounded subset of $\left(\mathbb{N}^{\uparrow N},<^{*}\right)$. Then

$$
(\forall k<\omega)(\exists x, y \in X)[\operatorname{osc}(x, y)=k] .
$$

Definition 12.4. An equivalence class $e \in D(x, y) / E(x, y)$ is said to be relatively large if

$$
|e|>|x(n)-y(n)| \text { for } n=\min (e) .
$$

Remark 12.5. Note that if $x<^{*} y$, one of the classes of $D(x, y) / E(x, y)$ is infinite, so in particular there is a relatively large class. In fact the proof of the previous result gives $x, y$ with $\operatorname{osc}(x, y)=k$ such that all classes of $D(x, y) / E(x, y)$ are relatively small except the last one which is infinite. So if we let

$$
\operatorname{osc}^{*}(x, y)=\operatorname{osc}(x\lceil n, y\lceil n),
$$

where $n$ is the minimum of the first relatively large class of $D(x, y) / E(x, y)$, we get the following reformulation of Theorem 12.3

\footnotetext{
${ }^{29}$ This result appears in [108] as two separate statements (Theorems 8.5 and 3.7, respectively) $\mathrm{TA} \Rightarrow \mathrm{A}$ and $\mathrm{A} \Rightarrow \mathfrak{b}=\aleph_{2}$, where $\mathrm{A}$ is a topological basis theorem.
} 
Theorem 12.6. Suppose $X$ is a totally ordered and unbounded subset of $\left(\mathbb{N}^{\uparrow N},<^{*}\right)$. Then

$$
(\forall k<\omega)(\exists x, y \in X)\left[\operatorname{osc}^{*}(x, y)=k\right] .
$$

Corollary 12.7. TA implies $\mathfrak{b}>\aleph_{1}$.

Proof. Choose a totally ordered and unbounded subset $X$ of $\left(\mathbb{N}^{\uparrow \mathbb{N}},<^{*}\right)$ of cardinality $\mathfrak{b}$. Consider the open graph $\mathcal{G}=(X, E)$, where we put $\{x, y\} \in E$ if $\operatorname{osc}(x, y)>0$ (or if $\operatorname{osc}^{*}(x, y)>0$ ).

Remark 12.8. It follows that under TA there is a sequence $\left\{x_{\xi}: \xi<\omega_{2}\right\} \subseteq \mathbb{N}^{\uparrow \mathbb{N}}$ such that $x_{\xi}<^{*} x_{\eta}$ whenever $\xi<\eta<\omega_{2}$.

Question 12.9. Given such an increasing sequence $\left(x_{\xi}: \xi<\omega_{2}\right)$, and assuming if necessary TA, when does the inner model $L\left[x_{\xi}: \xi<\omega_{2}\right]$ contain all the reals?

Theorem 12.10 ([110 $\left.]^{30}\right)$. Assume TA and fix an $<^{*}$-unbounded $<^{*}$-chain

$$
\left\{x_{\xi}: \xi<\omega_{2}\right\} \subseteq \mathbb{N}^{\uparrow \mathbb{N}} .
$$

Suppose further that $M \supseteq L\left[x_{\xi}: \xi<\omega_{2}\right]$ is an inner model of a sufficiently large fragment of ZFC with the property that every uncountable subset of $\omega_{1}$ contains an infinite subset belonging to $M$. Then $M$ contains all the reals.

Sketch of Proof. Let us say that an infinite set $X \subseteq \mathbb{N}^{\uparrow N}$ codes a real $r \in 2^{\mathbb{N}}$ if ${ }^{31}$

$$
(\forall x \neq y \in X)(\exists k \geq \Delta(x, y)(=\min D(x, y))) \operatorname{osc}^{*}(x, y)=\sum_{i=0}^{k} r(i) 2^{i} .
$$

Let $X=\left\{x_{\xi}: \xi<\omega_{2}\right\}$, fix a real $r \in 2^{\mathbb{N}} \backslash$ Fin, and assume TA.

Define $E_{r} \subseteq X^{2} \backslash \Delta$ by

$$
(x, y) \in E_{r} \Longleftrightarrow(\exists k \geq \Delta(x, y)) \operatorname{osc}^{*}(x, y)=\sum_{i=0}^{k} r(i) 2^{i} .
$$

Clearly $E_{r}$ is open. By the basic oscillation result, Theorem 12.6, we have $Y^{2} \cap E_{r} \neq$ $\emptyset$ for all unbounded $Y \subseteq X$, so in particular

$$
\operatorname{Chr}\left(X, E_{r}\right)>\aleph_{0} \text {. }
$$

Applying TA, we get an uncountable $Y \subseteq X$ coding $r$. Shrinking $Y$ we may assume that the closure $\bar{Y}$ is a compact subset of $\mathbb{N}^{\mathbb{N}}$. By our assumption about $M$ there is infinite $Y_{0} \subseteq Y$ such that $Y_{0} \in M$. By compactness of the closure of of $Y$, we have that $\sup \left\{\Delta(x, y): x, y \in Y_{0}, x \neq y\right\}=\infty$, It follows that $r \in M$.

Corollary 12.11. TA implies that the continuum is bounded by

$$
\min \left\{|\mathcal{F}|: \mathcal{F} \subseteq\left[\omega_{2}\right]^{\aleph_{0}} \text { and }\left(\forall X \in\left[\omega_{2}\right]^{\aleph_{1}}\right)(\exists D \in \mathcal{F}) D \subseteq X\right\} .
$$

Definition 12.12. For $Y \subseteq \mathbb{N}^{\uparrow \mathbb{N}}$, let

$$
R(Y)=\left\{r \in 2^{\mathbb{N}}: r \text { is coded by some uncountable } Z \subseteq Y\right\} .
$$

Problem 12.13. Assuming TA, is there a totally $<^{*}$-ordered $<^{*}$-unbounded chain $X=\left\{x_{\xi}: \xi<\omega_{2}\right\} \subseteq \mathbb{N}^{\mathbb{N}}$ such that $|R(X)| \leq \aleph_{2}$ ?

\footnotetext{
${ }^{30}$ This result was announced in print in [16], p. 168 in a slightly weaker formulation (that PFA fails in the Sacks forcing extension), but this is what the proof gives.

${ }^{31}$ Our original definition was slightly different (see [1], [119]), the current one is taken from 76].
} 


\section{The Open Graph Axiom and Gaps in $\mathcal{P}(\omega) /$ Fin}

Definition 13.1. For $a, b \subseteq \omega$, set

$$
a \perp b \text { if and only if } a \cap b \in \text { Fin. }
$$

For $\mathcal{A}, \mathcal{B} \subseteq \mathcal{P}(\omega)$, set

$$
\mathcal{A} \perp \mathcal{B} \text { if and only if }(\forall a \in \mathcal{A})(\forall b \in \mathcal{B}) a \perp b .
$$

For $\mathcal{A} \subseteq \mathcal{P}(\omega)$, set

$$
\mathcal{A}^{\perp}=\{b:(\forall a \in \mathcal{A}) b \cap a \in \text { Fin }\} .
$$

We say that $c \subseteq \omega$ separates $\mathcal{A}$ and $\mathcal{B}$ if and only if

$$
(\forall a \in \mathcal{A})(\forall b \in \mathcal{B})\left[a \subseteq^{*} c \& c \perp b\right] .
$$

We say that $\mathcal{C} \subseteq \mathcal{P}(\omega)$ separates $\mathcal{A}$ and $\mathcal{B}$ if and only if

$$
(\forall a \in \mathcal{A})(\forall b \in \mathcal{B})(\exists c \in \mathcal{C})\left[a \subseteq^{*} c \& c \perp b\right] .
$$

Example 13.2 (48, $[49])$. There exist two orthogonal $\omega_{1}$-chains $\mathcal{A}=\left\{a_{\xi}: \xi<\omega_{1}\right\}$ and $\mathcal{B}=\left\{b_{\xi}: \xi<\omega_{1}\right\}$ in the quotient algebra $\mathcal{P}(\omega) /$ Fin which cannot be separated. This is what is usually called Hausdorff's $\left(\omega_{1}, \omega_{1}\right)$-gap in $\mathcal{P}(\omega) /$ Fin.

Definition 13.3. An indexed family $\left\{\left(a_{i}, b_{i}\right): i \in I\right\} \subseteq \mathcal{P}(\omega) \times \mathcal{P}(\omega)$ is said to form a biorthogonal gap if

$$
\left(a_{i} \cap b_{j}\right) \cup\left(a_{j} \cap b_{i}\right)=\emptyset \text { iff } i=j
$$

Remark 13.4. While this is inspired by the Hausdorff condition on gaps, note that this notion corresponds to countable separation rather than separation. More precisely, note that a biorthogonal gap indexed by an uncountable set $I$ cannot be separated by a countable $\mathcal{C} \subseteq \mathcal{P}(\omega)$.

It turns out that TA has a considerable influence on the gap structure of the quotient algebra $\mathcal{P}(\omega)$ /Fin as the following fact shows.

Theorem 13.5 ([108]). Assume TA. For every pair $\mathcal{A}$ and $\mathcal{B}$ of downwards closed orthogonal families of subsets of $\omega$, we have that either

(1) some countable $\mathcal{C} \subseteq \mathcal{P}(\omega)$ separates $\mathcal{A}$ and $\mathcal{B}$, or

(2) there is an uncountable biorthogonal gap $\left\{\left(a_{i}, b_{i}\right): i \in I\right\} \subseteq \mathcal{A} \times \mathcal{B}$.

Proof. Let

$$
\mathcal{X}=\{(a, b) \in \mathcal{A} \times \mathcal{B}: a \cap b=\emptyset\}
$$

and let

$$
R=\left\{\left((a, b),\left(a^{\prime}, b^{\prime}\right)\right) \in \mathcal{X}^{2}:\left(a \cap b^{\prime}\right) \cup\left(b \cap a^{\prime}\right) \neq \emptyset\right\} .
$$

Then $\mathcal{G}=(\mathcal{X}, R)$ is an open graph, so applying TA, we have to consider the following two alternatives:

(1) If $\operatorname{Chr}(\mathcal{G}) \leq \aleph_{0}$ then there is a decomposition $\mathcal{X}=\bigcup_{n<\omega} \mathcal{X}_{n}$ such that $\left(\mathcal{X}_{n}\right)^{2} \cap R=\emptyset$ for all $n<\omega$. For $n<\omega$, let $c_{n}=\bigcup\left\{a:(\exists b)(a, b) \in \mathcal{X}_{n}\right\}$. Then the family $\mathcal{C}=\left\{c_{n}: n<\omega\right\}$ separates $\mathcal{A}$ and $\mathcal{B}$.

(2) If $\mathcal{K} \subseteq \mathcal{A} \otimes \mathcal{B}$ is a clique of $\mathcal{G}$, i.e. if $\mathcal{K}^{2} \backslash \Delta \subseteq R$ then any 1-1 enumeration $\mathcal{K}=\left\{\left(a_{i}, b_{i}\right): i \in I\right\}$ forms a biorthogonal gap.

Corollary 13.6. Assuming TA, the orthogonal $\mathcal{A}^{\perp}$ of any chain $\mathcal{A}$ of $\mathcal{P}(\omega) /$ Fin of cofinality $>\omega_{1}$ is countably generated. 
Remark 13.7. This result is sharp since under $\mathfrak{p}>\omega_{1}$, an assumption compatible with TA, the orthogonal of a chain $\mathcal{A}$ of $\mathcal{P}(\omega) /$ Fin of cofinality $\leq \omega_{1}$ is not countably generated.

Corollary 13.8 ([108]). TA implies $\mathfrak{b} \leq \aleph_{2}$.

Proof. If $\mathfrak{b}>\aleph_{2}$ then there is an $\omega_{2}$-chain in $\mathcal{P}(\omega) /$ Fin whose orthogonal is not countably generated. In fact this is true about any $\omega_{2}$-chain in $\mathcal{P}(\omega) /$ Fin with no least upper bound.

\section{The Open Graph Axiom and the Homomorphism Representation Problem for Quotient Algebras of the Form $\mathcal{P}(\mathbb{N}) / \mathcal{I}$}

This problem is about solving the following diagram

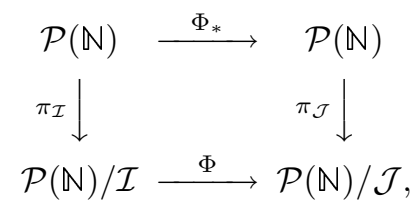

where $\mathcal{I}, \mathcal{J}$ are analytic ideals on $\mathbb{N}$ and $\pi_{\mathcal{I}}, \pi_{\mathcal{J}}$ are the corresponding quotient maps, $\Phi$ is a homomorphism we wish to study and $\Phi_{*}$ is its lifting. In other words, this is a representation theory of homomorphisms $\Phi: \mathcal{P}(\mathbb{N}) / \mathcal{I} \rightarrow \mathcal{P}(\mathbb{N}) / \mathcal{J}$ between quotient algebras over analytic ideals on $\mathbb{N}$ in terms of their liftings $\Phi_{*}: \mathcal{P}(\mathbb{N}) \rightarrow \mathcal{P}(\mathbb{N})$. Note that $\Phi_{*}$ may not be a homomorphism itself but when it is one gets a good solution to the lifting problem. Particularly desirable are the completely additive liftings $\Phi_{*}$, the maps $\Phi_{*}: \mathcal{P}(\mathbb{N}) \rightarrow \mathcal{P}(\mathbb{N})$ that preserve even infinitary Boolean operations. Note that every such $\Phi_{*}$ has the form $\Phi_{*}(x)=h^{-1}(x)$ for some map $h: \mathbb{N} \rightarrow \mathbb{N}$ witnessing the Rudin-Keisler reduction $\mathcal{J} \leq_{\mathrm{RK}} \mathcal{I}{ }^{32}$ Note also that such maps $\Phi_{*}$ are continuous suggesting us to consider also topological requirements on the liftings. This has turned out to be the right approach as first shown in the case of $\mathcal{I}=\mathcal{J}=$ Fin by the ground-breaking work 90 .

Theorem 14.1 ( 61, 90, 132]). If a homomorphism $\Phi: \mathcal{P}(\mathbb{N}) /$ Fin $\rightarrow \mathcal{P}(\mathbb{N}) /$ Fin has a Baire-measurable (or equivalently continuous) lifting then it also has a completely additive lifting, so its kernel is an ideal generated over Fin by a single subset of $\mathbb{N}$.

That this remains true for arbitrary homomorphism $\Phi: \mathcal{P}(\mathbb{N}) /$ Fin $\rightarrow \mathcal{P}(\mathbb{N}) /$ Fin in some model of set was also first shown in 90 but the introduction of TA in this area was made in [133 and 62 allowing us to have the following result.

Theorem 14.2 (62, [122, [133]). Assume TA. If $\mathcal{I}$ is a non-atomic analytic ideal on $\mathbb{N}$ then $\mathcal{P}(\mathbb{N}) / \mathcal{I}$ is not isomorphic to a subalgebra of $\mathcal{P}(\mathbb{N}) /$ Fin.

The ground-breaking work for the general theory was made in 62 building on a series of previous papers [59, 60, ,61].

Theorem 14.3 ([62]). TA implies $\mathcal{P}(\mathbb{N}) / \mathcal{Z}_{0} \nsucceq \mathcal{P}(\mathbb{N}) / \mathcal{Z}_{\text {log }} \cdot{ }^{33}$

This suggested two directions of study in this area that are based on two different theories:

\footnotetext{
${ }^{32}$ In other words, for an arbitrary set $Y \subseteq \mathbb{N}$, we have that $Y \in \mathcal{J}$ if and only if $h^{-1}(Y) \in \mathcal{I}$.

${ }^{33}$ Here, $\mathcal{Z}_{0}=\left\{X \subseteq \mathbb{N}: \lim _{n \rightarrow \infty} \frac{|A \cap n|}{n}=0\right\}$, and $\mathcal{Z}_{\log }=\left\{X \subseteq \mathbb{N}: \lim _{n \rightarrow \infty} \frac{\sum_{k \in A \cap n} \frac{1}{k+1}}{\ln n}=0\right\}$.
} 


\begin{tabular}{c|c} 
ZFC & ZFC+MA+TA \\
\hline homomorphisms with continuous liftings & arbitrary homomorphisms
\end{tabular}

In fact, there is a close connection between these two directions which is supported by the fact that TA has the power of reducing problems about arbitrary homomorphisms to problems about homomorphisms with Baire-measurable liftings. The study of the special case of analytic P-ideals was first suggested in [122] inspired by the following two results.

Theorem 14.4 (122]). Suppose $\Phi: \mathcal{P}(\mathbb{N}) / \mathcal{I} \rightarrow \mathcal{P}(\mathbb{N}) / \mathcal{J}$ is an isomorphic embedding with a continuous lifting. If $\mathcal{J}$ is an analytic P-ideal then so is $\mathcal{I}$.

Theorem 14.5 ([122]). Assume TA and that $\mathcal{I}$ and $\mathcal{J}$ are analytic ideals such that $\mathcal{P}(\mathbb{N}) / \mathcal{I}$ is embeddable into $\mathcal{P}(\mathbb{N}) / \mathcal{J}$. If $\mathcal{J}$ is a P-ideal then so is $\mathcal{I}$.

This turned out to be a fruitful approach as shown in another ground-breaking work 31] where it is proved that in the case of analytic P-ideals $\mathcal{I}$ and $\mathcal{J}$, the lifting problem from [122] is equivalent to a finite Ulam-stability problem which has a positive solution if the lower-semicontinuous submeasure $\varphi: \mathcal{P}(\mathbb{N}) \rightarrow[0, \infty)$ giving $\mathcal{J}$ as (see 94)

$$
\mathcal{J}=\left\{X \subseteq \mathbb{N}: \lim _{n \rightarrow \infty} \varphi(X \backslash n)=0\right\}
$$

is non-pathological. Here are two sample results from this extensive piece of work.

Theorem 14.6 ([31]). Assume MA+TA. Let $\mathcal{I}$ and $\mathcal{J}$ be two analytic P-ideals given by non-pathological submeasures. Then every isomorphism between $P(\mathbb{N}) / \mathcal{I}$ and $\mathcal{P}(\mathbb{N}) / \mathcal{J}$ has a completely additive lifting.

Corollary 14.7 ([31]). Assuming MA+TA,

$$
\mathcal{P}(\mathbb{N}) / \mathcal{I}_{1 / n} ¥ \mathcal{P}(\mathbb{N}) / \mathcal{I}_{1 / \sqrt{n}} \cdot 34
$$

The following result shows that the axiomatic assumption of MA+TA is in some sense optimal and points out the close relationship between the axiomatic and mathematical work in this area.

Theorem 14.8 (31]). The following conditions are equivalent for every pair of analytic $P$-ideals $\mathcal{I}$ and $\mathcal{J}{ }^{35}$

(1) The theory ZFC is insufficient for proving $\mathcal{P}(\mathbb{N}) / \mathcal{I} \cong \mathcal{P}(\mathbb{N}) / \mathcal{J}$;

(2) $\mathcal{P}(\mathbb{N}) / \mathcal{I}$ and $\mathcal{P}(\mathbb{N}) / \mathcal{J}$ are not isomorphic via a continuous lifting.

(3) The theory $\mathrm{ZFC}+\mathrm{MA}+\mathrm{TA}$ is sufficient for proving $\mathcal{P}(\mathbb{N}) / \mathcal{I} \nsucceq \mathcal{P}(\mathbb{N}) / \mathcal{J}$;

Remark 14.9. Following the standard Christensen approach ([18]) to formulate the notion of non-pathological submeasure in terms of the Fubini property this work was further refined in 63] allowing the theory of lifting to be extended to a quite large class of analytic ideals on $\mathbb{N}$. It should be noted however that there are no known restrictions on analytic (P-)ideals in the following special case of Problem 1 of 122 .

Problem 14.10. Suppose $\Phi: \mathcal{P}(\mathbb{N}) / \mathcal{I} \rightarrow \mathcal{P}(\mathbb{N}) / \mathcal{J}$ is an isomorphism between two quotient algebras over analytic (P-)ideals $\mathcal{I}$ and $\mathcal{J}$ on $\mathbb{N}$ represented by a continuous lifting $\Phi^{*}: \mathcal{P}(\mathbb{N}) \rightarrow \mathcal{P}(\mathbb{N})$. Does $\Phi$ has a completely additive lifting?

\footnotetext{
${ }^{34}$ Recall that, $\mathcal{I}_{1 / n}=\left\{X \subseteq \mathbb{N}: \sum_{n \in X} \frac{1}{n}<\infty\right\}$, and $\mathcal{I}_{1 / \sqrt{n}}=\left\{X \subseteq \mathbb{N}: \sum_{n \in X} \frac{1}{\sqrt{n}}<\infty\right\}$.

${ }^{35}$ The provability here could be taken relative to, say, $\beta$-logic, or $\Omega$-logic (see [137]).
} 
Of course, similar questions can be asked for arbitrary isomorphisms assuming TA or one of its natural strengthenings such as PFA or MM. Note that (as also indicated in Problem 1 of [122]) in this case one should consider more general surjective homomorphisms $\Phi: \mathcal{P}(\mathbb{N}) /$ Fin $\rightarrow \mathcal{P}(\mathbb{N}) / \mathcal{J}$. For more about the work in this direction the reader is referred to the articles [34] and 33.

\section{The Open Graph Axiom and the Automorphism Representation Problem for the Calkin Algebra}

In this section we present recent advances towards the automorphism representation problem in the context of noncommutative algebra or more precisely in the context of $\mathrm{C}^{*}$-algebra. So let us recall some standard notation and definitions from this area referring the reader to some standard text such as 80 for further background.

Definition 15.1. For a separable infinite-dimensional complex Hilbert space $H$, we associate the following standard objects,

$\mathcal{B}(H)$ : The $\mathrm{C}^{*}$-algebra of bounded operators on $H$.

$\mathcal{K}(H) \subseteq \mathcal{B}(H)$ : The ideal of compact operators

$\mathcal{C}(H)=\mathcal{B}(H) / \mathcal{K}(H)$ : The Calkin algebra

Recall also that every separable $\mathrm{C}^{*}$-algebra is isomorphic to a subalgebra of $\mathcal{B}(H)$. An automorphism $\Phi$ of a $\mathrm{C}^{*}$-algebra is inner if there is a unitary $u$ such that

$$
(\forall a) \Phi(a)=u a u^{*}
$$

It is easily seen that every automorphism of $\mathcal{B}(H)$ is inner but the problem in this context is about the following diagram

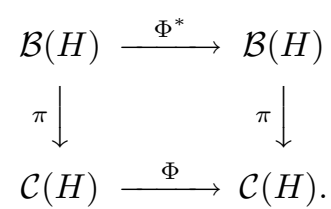

More precisely, one would like to find conditions on representation $\Phi^{*}$ for a given automorphism $\Phi$ of the Calkin algebra that guarantee $\Phi$ to be inner. In particular one would like to know the answer to the following question.

Question 15.2 ([15]). Is there an outer automorphism of the Calkin algebra?

Theorem 15.3 ([81]). $\mathrm{CH}$ implies that there is an outer automorphism of the Calkin algebra.

Theorem 15.4 (35]). TA implies all automorphisms of the Calkin algebra are inner.

The proof uses several ideas developed in the context of commutative theory (see the previous section) but it is of course considerably more subtle. We mention only some of the new features that appear in the proof. For an orthogonal decomposition $\vec{J}=\left(J_{n}\right)_{n<\omega}$ of $H$ into finite-dimensional subspaces, let

$$
\begin{aligned}
\mathcal{D}(\vec{J}) & =\left\{a \in \mathcal{B}(H):(\forall n) a\left[J_{n}\right] \subseteq J_{n}\right\} \\
\mathcal{K}(\vec{J}) & =\mathcal{D}(\vec{J}) \cap \mathcal{K}(H) \\
\mathcal{C}(\vec{J}) & =\mathcal{D}(\vec{J}) / \mathcal{K}(\vec{J}) .
\end{aligned}
$$


It turns out that an automorphism $\Phi$ of the Calkin Algebra $\mathcal{C}(H)$ is uniquely determined by its restrictions to subalgebras of the form $\mathcal{C}(\vec{J})$ so it is natural to study these restrictions. The following result shows that axioms like TA will be necessary in this study.

Theorem 15.5 ([35]). Using $\mathrm{CH}$ one can build an automorphism of $\mathcal{C}(H)$ that is not inner but it is inner on $\mathcal{C}(\vec{J})$ for every finite dimensional orthogonal decomposition $\vec{J}=\left(J_{n}\right)$ of $H$.

Fix an automorphism $\Phi$ of the Calkin algebra $\mathcal{C}(H)$. The proof of Theorem 15.4 splits naturally into two parts which resemble the corresponding parts in the commutative case, the theory of gaps in the quotient structures. In particular the second lemma below follows from a more informative fact which shows that under TA every 'coherent family of unitaries is trivial' which has its direct analog in the noncommutative case.

Lemma 15.6 (35). TA implies that $\Phi$ is inner on $\mathcal{C}(\vec{J})$ for each finite dimensional orthogonal decomposition $\vec{J}=\left(J_{n}\right)$ of $H$.

Lemma 15.7 (35]). TA implies that if an automorphism of $\mathcal{C}(H)$ is inner on $\mathcal{C}(\vec{J})$ for each finite dimensional orthogonal decomposition $\vec{J}=\left(J_{n}\right)$ of $H$ then it must be inner on $\mathcal{C}(H)$.

As in the commutative theory Theorem 15.4 has its ZFC version stated as follows.

Theorem 15.8 (35). An automorphism $\Phi$ of the Calkin algebra $\mathcal{C}(H)$ is inner if and only if it has a representation $\Phi^{*}: \mathcal{B}(H) \rightarrow \mathcal{B}(H)$ whose restriction to the unit ball of $\mathcal{B}(H)$ is measurable relative to the $\sigma$-algebra generated by analytic sets ${ }^{36}$

\section{The Open Graph Axiom and Cofinalities of Banach Spaces}

Recall the following well known problem from Banach space theory (see, for example, [78]

Problem 16.1 (Separable Quotient Problem). Does every infinite-dimensional Banach space $X$ have a separable infinite-dimensional quotient $X / Y$ ?

This problem is related to the following purely set-theoretic notion.

Definition 16.2. The cofinality, $\operatorname{cf}(X)$, of a given Banach space $X$ is the minimal ordinal $\gamma$ for which there is an increasing chain $X_{\xi} \quad(\xi<\gamma)$ of proper closed subspaces whose union $\bigcup_{\xi<\gamma} X_{\xi}$ is dense in $X$.

Problem 16.3 (Equivalent Reformulation of the Separable Quotient Problem). Does every infinite-dimensional Banach space have cofinality $\omega$ ?

Theorem 16.4 (127]). Assume TA. Then $\omega$ and $\omega_{1}$ are the only two possible values for the cofinality $\operatorname{cf}(X)$ of a given infinite-dimensional Banach space $X$.

Sketch of Proof. We consider several cases.

Case 1: $l_{1} \hookrightarrow X^{*}$. Then applying a combination of a result of Johnson-Rosenthal and a result of Hagler-Johnson one finds closed $Y \subseteq X$ with $X / Y$ infinite-dimensional and separable, and therefore $\operatorname{cf}(X)=\omega$.

\footnotetext{
${ }^{36}$ Here, we take $\mathcal{B}(H)$ with its strong operator topology so that its unit ball is Polish.
} 
Case 2: $l_{1} \leftrightarrow X^{*}$. In this case from the well-known work of about Baire-class-one functions (86] and [14]) one can deduce the following property of the dual ball $B_{X^{*}}$ :

Every sequence $\left(x_{n}^{*}\right)$ of elements of $B_{X^{*}}$ has a convergent subsequence relative to the weak (and therefore weak ${ }^{*}$ ) topology of $B_{X^{*}}$.

In fact, every countable $D \subseteq B_{X^{*}}$ is sequentially dense in its closure (even inside $\left.B_{X^{* * *}}\right)$.

Case 2.1: $l_{1} \hookrightarrow X^{*}$ and there is countable $D \subseteq B_{X^{*}}$ such that $\bar{D}$ has a non- $G_{\delta^{-}}$ point.

$$
\begin{gathered}
D=\left\{f_{n}: n<\omega\right\} \\
0^{*} \in \bar{D} \text { not } G_{\delta} \text { relative to } \bar{D} . \\
\mathcal{A}=\left\{a \subseteq \omega: 0^{*} \notin \overline{\left\{f_{n}: n \in a\right\}}\right\} \\
\mathcal{B}=\mathcal{A}^{\perp}=\{a \subseteq \omega:(\forall a \in \mathcal{A}) b \cap a \in \mathrm{Fin}\} \\
\mathcal{A}_{0}=\left\{a \subseteq \omega:\left(f_{n}\right)_{n \in a} \text { converges to a point } \neq 0^{*}\right\}
\end{gathered}
$$

Note 16.5. $\mathcal{A}_{0}$ is a dense subset of $\mathcal{B}$, i.e., for every infinite $b \in \mathcal{B}$ there is infinite $a \in \mathcal{A}_{0}$ such that $a \subseteq b$.

Claim 1. No countable subset of $\mathcal{P}(\omega)$ separates $\mathcal{A}$ and $\mathcal{B}$, or equivalently, no countable subset of $\mathcal{P}(\omega)$ separates $\mathcal{A}_{0}$ and $\mathcal{B}$.

So applying TA, we get a biorthogonal gap $\left\{\left(a_{\xi}, b_{\xi}\right): \xi<\omega\right\} \subseteq \mathcal{A}_{0} \times \mathcal{B}$. For $\xi<\omega_{1}$, let

$$
g_{\xi}=\lim _{n \in a_{\xi}} f_{n}
$$

Finally, for $\alpha<\omega_{1}$, let

$$
X_{\alpha}=\left\{x \in X:(\forall \xi \geq \alpha) g_{\xi}(x)=0\right\} .
$$

Then $X_{\alpha} \varsubsetneqq X, X_{\alpha} \subseteq X_{\beta}$ for $\alpha<\beta<\omega_{1}$, and $X=\bigcup_{\alpha<\omega_{1}} X_{\alpha}$. It follows that $\operatorname{cf}(X) \leq \omega_{1}$.

Case 2.1*: $l_{1} \hookrightarrow X^{*}$ and there is countable $D \subseteq B_{X^{*}}$ whose closure contains a closed non- $G_{\delta}$-subset $F$. Set

$$
\begin{aligned}
& \mathcal{A}=\left\{a \subseteq \omega:\left(f_{n}\right)_{n \in a} \text { converges to a point of } F\right\} \\
& \mathcal{B}=\left\{b \subseteq \omega:\left(f_{n}\right)_{n \in b} \text { converges to a point outside } F\right\}
\end{aligned}
$$

Claim 2. No countable subset of $\mathcal{P}(\omega)$ separates $\mathcal{A}$ and $\mathcal{B}$.

Applying again TA, we get a biorthogonal gap $\left\{\left(a_{\xi}, b_{\xi}\right): \xi<\omega_{1}\right\} \subseteq \mathcal{A} \times \mathcal{B}$. For $\xi<\omega_{1}$, let

$$
g_{\xi}=\lim _{n \in a_{\xi}} f_{n}, \quad h_{\xi}=\lim _{n \in b_{\xi}} f_{n}
$$

and

For $\alpha<\omega_{1}$, let

$$
e_{\xi}=g_{\xi}-f_{\xi}\left(\neq 0^{*} !\right)
$$

$$
X_{\alpha}=\left\{x \in X:(\forall \xi \geq \alpha) e_{\xi}(x)=0\right\} .
$$

Then $X_{\alpha} \neq X, X_{\alpha} \subseteq X_{\beta}$ for $\alpha<\beta<\omega_{1}$, and $X=\bigcup_{\alpha<\omega_{1}} X_{\alpha}$. So again, we get that $\operatorname{cf}(X) \leq \omega_{1}$.

Case 2.2: There is countable $D \subseteq B_{X^{*}}$ whose closure $K=\bar{D}$ is not secondcountable though all of its closed subsets are relatively $G_{\delta}$. For $f \in K$, let $V_{n}(f)$ 
$(n<\omega)$ be a fixed neighborhood base of $f$ in $K$. Then using the assumption that $K$ is not metrizable we can find a sequence $\left(f_{\alpha}, g_{\alpha}\right) \alpha<\omega_{1}$ of pairs of distinct elements of $K$ such that if $\alpha<\beta$ and $n<\omega$ then the open sets $V_{n}\left(f_{\alpha}\right)$ and $V_{n}\left(g_{\alpha}\right)$ do not separate the points $f_{\beta}$ and $g_{\beta}$. Let

$$
h_{\beta}=f_{\beta}-g_{\beta}\left(\beta<\omega_{1}\right) .
$$

Then one can easily check that

$$
(\forall x \in X)\left\{\beta<\omega_{1}: h_{\beta}(x)=0\right\} \text { is countable. }
$$

So, as before, if for $\alpha<\omega_{1}$, we set

$$
X_{\alpha}=\left\{x \in X:(\forall \beta \geq \alpha) h_{\beta}(x)=0\right\}
$$

we get that $X_{\alpha} \neq X, X_{\alpha} \subseteq X_{\beta}$ for $\alpha<\beta<\omega_{1}$, and $X=\bigcup_{\alpha<\omega_{1}} X_{\alpha}$. It follows that $\operatorname{cf}(X) \leq \omega_{1}$ also in this case.

Case 2.3: For every countable $D \subseteq B_{X^{*}}$, the closure $K_{D}=\bar{D}$ is secondcountable. Choose an increasing sequence $D_{\alpha}\left(\alpha<\omega_{1}\right)$ of countable subsets of $B_{X^{*}}$ such that

$$
\overline{D_{\alpha}} \varsubsetneqq \overline{D_{\alpha+1}} \text { for all } \alpha<\omega_{1} \text {. }
$$

Let

$$
K=\overline{\bigcup_{\alpha<\omega_{1}} D_{\alpha}}
$$

Case 2.3.1: $K \neq \bigcup_{\alpha<\omega_{1}} \overline{D_{\alpha}}$. Then no point of $K \backslash \bigcup_{\alpha<\omega_{1}} \overline{D_{\alpha}}$ is in the closure of a countable subset of $\bigcup_{\alpha<\omega_{1}} \overline{D_{\alpha}}$, so by $([58])$, we could find a sequence $\left\{f_{\xi}: \xi<\right.$ $\left.\omega_{1}\right\} \subseteq \bigcup_{\alpha<\omega_{1}} \overline{D_{\alpha}}$ converging to a point $g \in K \backslash \bigcup_{\alpha<\omega_{1}} \overline{D_{\alpha}}$. Let

$$
h_{\xi}=f_{\xi}-g\left(\xi<\omega_{1}\right) \text {. }
$$

Then as before, if for $\alpha<\omega_{1}$, we set

$$
X_{\alpha}=\left\{x \in X:(\forall \xi \geq \alpha) h_{\xi}(x)=0\right\}
$$

we get that $X_{\alpha} \neq X, X_{\alpha} \subseteq X_{\beta}$ for $\alpha<\beta<\omega_{1}$, and $X=\bigcup_{\alpha<\omega_{1}} X_{\alpha}$. It follows that $\operatorname{cf}(X) \leq \omega_{1}$ in this case as well.

Case 2.3.2: $K=\bigcup_{\alpha<\omega_{1}} \overline{D_{\alpha}}$. By our assumption each $\overline{D_{\alpha}}$ is second countable, so the compactum $K$, while non-metrizable, has a basis of size $\aleph_{1}$. So fix a basis $V_{\xi}\left(\xi<\omega_{1}\right)$ for $K$ consisting of co-zero subsets of $K$. Then we can find a sequence $\left(f_{\alpha}, g_{\alpha}\right)\left(\alpha<\omega_{1}\right)$ of pairs of disctinct elements of $K$ such that

$$
\left(\forall \xi<\alpha<\omega_{1}\right) f_{\alpha} \in V_{\xi} \Leftrightarrow g_{\alpha} \in V_{\xi} .
$$

Let

$$
h_{\alpha}=f_{\alpha}-g_{\alpha}\left(\alpha<\omega_{1}\right) .
$$

Since $V_{\xi}\left(\xi<\omega_{1}\right)$ is a basis for $K$, it follows that

$$
(\forall x \in X)\left\{\alpha<\omega_{1}: h_{\alpha}(x)=0\right\} \text { is countable. }
$$

So if for $\alpha<\omega_{1}$, we set

$$
X_{\alpha}=\left\{x \in X:(\forall \beta \geq \alpha) h_{\beta}(x)=0\right\}
$$

we get that $X_{\alpha} \neq X, X_{\alpha} \subseteq X_{\beta}$ for $\alpha<\beta<\omega_{1}$, and $X=\bigcup_{\alpha<\omega_{1}} X_{\alpha}$. So we get $\operatorname{cf}(X) \leq \omega_{1}$ in this case as well. 


\section{The Open Graph Axiom and the Theory of Analytic Gaps}

The axiom TA is one of those set theoretic principles that can sometimes be removed from intended applications giving us results that do not rely on any addition axiom at all. In this section we give some examples of results obtained this way. They concern the theory of definable gaps in the quotient structures such as $\mathcal{P}(\mathbb{N}) /$ Fin or $\mathbb{N}^{\mathbb{N}} /$ Fin.

Example 17.1. Let Seq be the tree of finite sequences of integers ordered by end-extension. Let $\mathcal{C} \mathcal{H}_{\text {Seq }}$ be the family of all chains of Seq and let $\mathcal{I} \mathcal{S}_{\text {Seq }}$ be the family of subsets of Seq that are immediate successors of some node of Seq, i.e., the downwards closure of the family $\left\{t^{\frown} n: n \in \mathbb{N}\right\},(t \in$ Seq).

Note that $\mathcal{C} \mathcal{H}_{\text {Seq }}$ and $\mathcal{I} \mathcal{S}_{\text {Seq }}$ are two orthogonal analytic families of subsets of the tree Seq which can't be separated and, in fact, the family $\mathcal{C} \mathcal{H}_{\text {Seq }}$ of chains is not countably generated in the orthogonal $\left(\mathcal{I} \mathcal{S}_{\text {Seq }}\right)^{\perp}$. The following result shows that this is a canonical object in this category of gaps.

Theorem 17.2 ([118]). Suppose that $\mathcal{A}$ and $\mathcal{B}$ are two orthogonal downwards closed families of subsets of $\mathbb{N}$ and that $\mathcal{A}$ is analytic. Then either,

(1) $\mathcal{A}$ is countably generated in $\mathcal{B}^{\perp}$, or

(2) there is a 1-1 map $\varphi:$ Seq $\rightarrow \mathbb{N}$ such that $\varphi$ " $\mathcal{H H}_{\text {Seq }} \subseteq \mathcal{A}$ and $\varphi^{\prime \prime} \mathcal{I} \mathcal{S}_{\text {Seq }} \subseteq \mathcal{B}$.

Corollary 17.3 ([118]). If an analytic ideal $\mathcal{I}$ on $\mathbb{N}$ has the diagonal sequence property ${ }^{37}$ then its orthogonal $\mathcal{I}^{\perp}$ is countably generated.

Proof. We show that $\mathcal{I}^{\perp}$ is an $F_{\sigma}$ ideal and then the conclusion follows by applying the theorem to the pair $\left(\mathcal{I}^{\perp}, \mathcal{I}\right)$ of analytic orthogonal families ${ }^{38}$ Let

$$
\mathcal{X}=\left\{K \in \mathcal{K}\left(2^{\mathbb{N}}\right):(\exists b \in \mathcal{I})(\forall x \in K) b \cap x \notin \mathrm{FIN}\right\} .
$$

Note that $\mathcal{A}$ is an analytic subset of the space $\mathcal{K}(G)$ of compact subsets of $G=$ $\mathcal{P}(\mathbb{N}) \backslash I^{\perp}$. Since we need to prove that $G$ is a $G_{\delta}$ subset of the Cantor cube, applying Christensen's theorem ([17]), it suffices to show that every countable compact subset $K$ of $G$ belongs to $\mathcal{X}$. For each $x \in K$ fix $a_{x} \in \mathcal{I}$ such that $x \cap a_{x}$ is infinite. Since $K$ is countable, and since $\mathcal{I}$ has the diagonal sequence property, there is $b \in \mathcal{I}$ such that $x \cap a_{x} \cap b$ is infinite for all $x \in K$. It follows that $b \cap x$ is infinite for all $x \in K$. This shows that $K \in \mathcal{X}$.

Corollary 17.4 ([118]). Suppose $\mathcal{I}$ and $\mathcal{J}$ are two orthogonal P-ideals on $\mathbb{N}$. If one of them is analytic then they can be separated.

Remark 17.5. So Hausdorff $\left(\omega_{1}, \omega_{1}\right)$-gaps in $\mathcal{P}(\mathbb{N}) /$ Fin (see, 48, 49]) are highly non-analytic!

Let us now examine the case when both of the two orthogonal families are definable, or in other words, look for the symmetric version of the analytic gap theorem.

\footnotetext{
${ }^{37}$ An ideal $\mathcal{I}$ has the diagonal sequence property if for every $\left\{a_{n}: n<\omega\right\} \subseteq \mathcal{I} \backslash$ Fin there is $b \in \mathcal{I}$ such that $b \cap a_{n} \neq \emptyset$ for all $n<\omega$. Clearly, every P-ideal has this property.

${ }^{38}$ In fact, we only need the consequence saying that if $\mathcal{A}$ and $\mathcal{B}$ are orthogonal ideals such that $\mathcal{A}$ is analytic and $\mathcal{B}$ has the diagonal sequence property then $\mathcal{A}$ is countably generated in $\mathcal{B}^{\perp}$.
} 
Example 17.6. Let $\mathrm{Seq}_{2}$ be the tree of finite sequences of 0's and 1's. We identify the Cantor space $2^{\mathbb{N}}$ with the collection of all infinite branches of this tree. For $x \in 2^{\mathbb{N}}$ and $i<2$, let

$$
a_{x}^{i}=\left\{t \in \mathrm{Seq}_{2}: t \sqsubseteq x \text { and } x(|t|)=i\right\} .
$$

Fir $i<2$, let $\mathcal{A}_{\mathrm{Seq}_{2}}^{i}=\left\{a_{x}^{i}: x \in 2^{\mathbb{N}}\right\}$. Then $\mathcal{A}_{\mathrm{Seq}_{2}}^{0}$ and $\mathcal{A}_{\mathrm{Seq}_{2}}^{1}$ are two orthogonal analytic (in fact, perfect) families of chains of the tree $\mathrm{Seq}_{2}$ which form a gap which is not even countably separated. In fact using our terminology from Section 13 above, $\mathcal{A}_{\mathrm{Seq}_{2}}^{0}$ and $\mathcal{A}_{\mathrm{Seq}_{2}}^{1}$ form a perfect biorthogonal gap.

The following result shows that this is a canonical object in this category of analytic gaps.

Theorem 17.7 ([118]). Suppose that $\mathcal{A}$ and $\mathcal{B}$ are two orthogonal analytic families of subsets of $\mathbb{N}$ closed under finite changes. Then either,

(1) $\mathcal{A}$ and $\mathcal{B}$ are countably separated, or

(2) there is a 1-1 map $\varphi: \mathrm{Seq}_{2} \rightarrow \mathbb{N}$ such that $\varphi$ " $\mathcal{A}_{\mathrm{Seq}_{2}}^{0} \subseteq \mathcal{A}$ and $\varphi$ " $\mathcal{A}_{\mathrm{Seq}_{2}}^{1} \subseteq \mathcal{B}$.

A generalization of this result to more than two orthogonal analytic families is given in 4. Let us mention a typical application of this result.

Theorem 17.8 ([123]). Suppose $K$ is a separable compact subset of Baire-class1 functions on some complete metric space $X$ which in the topology of pointwise convergence on $X$ has the constant zero function $\overline{0}$ as a non-G $G_{\delta}$ point. Then there is a 1-1 Souslin-measurable map $\varphi: 2^{\mathbb{N}} \rightarrow K$ whose range is weakly null ${ }^{39}$

We mention an interesting recent application of this result to the separable quotient problem.

Theorem 17.9 ([3]). Every dual Banach space $X$ has a separable quotient.

Sketch. Let $X=Y^{*}$ and replacing $X$ and $Y$ we my assume $Y$ is separable and that $X$ is not. We may also assume that $\ell_{1}$ does not embed into $Y$ since otherwise $\ell_{\infty}$ would be a quotient of $X$. Thus the unit ball $B_{Y^{* *}}=B_{X^{*}}$ is a separable compact set of Baire-class-1 functions on $X$ (see [79]) with $0^{* *}$ a non- $G_{\delta}$ point. So Theorem 17.8 applies giving us a 1-1 Souslin-measurable $\varphi: 2^{\mathbb{N}} \rightarrow B_{X^{*}}$ whose range is weakly null. We may assume that the range of $\phi$ is semi-normalized. Now note that for every positive integer $k$ and $C>1$ the set of all 1-1 sequences $\left(a_{i}\right)_{i<k}$ of elements of $2^{\mathbb{N}}$ for which the corresponding sequence $\left(\phi\left(a_{i}\right)\right)_{i<k}$ is $C$-unconditiona ${ }^{40}$ is comeager in $\left(2^{\mathbb{N}}\right)^{k}$. So by Mycielsky's theorem $([129] ; 6.40)$, we get a perfect unconditional sequence and in particular an infinite unconditional sequence in $X^{*}$. So by the results of Johnson and Rosenthal ([57], 45]), $X$ has a separable quotient.

As the reader could have already guessed, if $D$ is a countable dense subset of the compactum $K$ from the hypothesis of Theorem 17.8 one obtains $\phi$ from the alternative (2) of Theorem 17.7. where $\mathcal{A}$ is the set of all subsets of $D$ which don't accumulate to $\overline{0}$ and where $\mathcal{B}$ is its orthogonal, i.e., the set of all subsets of $D$ which converge to $\overline{0}$. It is not hard to see that (1) fails for the gap formed by $\mathcal{A}$ and $\mathcal{B}$. Note however that Theorem 17.7 does not quite apply here since $\mathcal{B}$ is not analytic.

\footnotetext{
${ }^{39}$ In other words, for every $x \in X$ and $\varepsilon>0$ the set $\left\{a \in 2^{\mathbb{N}}:|\phi(a)(x)| \geq \varepsilon\right\}$ is finite.

${ }^{40}$ Recal that a sequence $\left(x_{i}\right)_{i \in I}$ of elements of some normed space $(N,\|\cdot\|)$ is $C$-unconditional if $\left\|\sum_{i \in F} x_{i}\right\| \leq C\left\|\sum_{i \in G} x_{i}\right\|$ for every pair $F \subseteq G$ of finite subsets of $I$.
} 
Examining the proof of Theorem 17.8 one discovers the reason of why we still have the alternative (2) for these $\mathcal{A}$ and $\mathcal{B}$ and gets to the following result.

Theorem 17.10 (24]). Suppose that $\mathcal{A}$ and $\mathcal{B}$ are two orthogonal families of subsets of $\mathbb{N}$ closed under finite changes, that $\mathcal{A}$ is analytic, that $\mathcal{B}$ is Souslin-measurable and that $\mathcal{B}$ has the weak diagonal sequence property ${ }^{41}$ Then either,

(1) $\mathcal{A}$ and $\mathcal{B}$ are countably separated, or

(2) there is a 1-1 map $\varphi: \mathrm{Seq}_{2} \rightarrow \mathbb{N}$ such that $\varphi$ " $\mathcal{A}_{\mathrm{Seq}_{2}}^{0} \subseteq \mathcal{A}$ and $\varphi " \mathcal{A}_{\mathrm{Seq}_{2}}^{1} \subseteq \mathcal{B}$.

Applications of the gap dichotomies to the study of diagonal sequence properties in the class of analytic topological spaces is given in [130.

\section{Open Graphs on Polish Spaces}

In this section we examine this phenomenon in some details by considering the following abstract property drown from the statement of TA.

Definition 18.1. Given a topological space $X$ we let $\mathrm{TA}(X)$ denote the statement that for every open graph $\mathcal{G}=(X, R)$ on $X$ either

(1) $\operatorname{Chr}(\mathcal{G}) \leq \aleph_{0}$, or

(2) $\mathcal{K}_{\aleph_{1}} \leq \mathcal{G}$.

The point of this definition is to isolate spaces $X$ for which $\mathrm{TA}(X)$ is simply true. For example, we have the following simple result ${ }^{42}$

Proposition 18.2. $\mathrm{TA}(X)$ is true for $X=\mathbb{N}^{\mathbb{N}}$.

Moreover, we have the following preservation result for this property.

Lemma 18.3. TA $(X)$ implies $\mathrm{TA}(Y)$ whenever $Y$ is a continuous image of $X$.

Proof. Fix a continuous onto map $f: X \rightarrow Y$ and an open graph $\mathcal{G}=(Y, R)$ on $Y$. Define

$$
S=\left\{(x, y) \in X^{2}: f(x) \neq f(y) \&(f(x), f(y)) \in R\right\} .
$$

Then $\mathcal{H}=(X, S)$ is an open graph on $X$ and an application of $\mathrm{TA}(X)$ to $\mathcal{H}$ gives us the conclusions of $\mathrm{TA}(Y)$ for the graph $\mathcal{G}$.

Corollary 18.4. $\mathrm{TA}(X)$ is true for every analytic space $X$, and so in particular, for every Polish space $X$.

Remark 18.5. In fact, the second alternative of $\mathrm{TA}(X)$ can be strengthened as follows:

(2) $* K_{2^{\mathrm{N}}} \leq_{c} \mathcal{G}$.

Let us denote this strong form of $\mathrm{TA}(X)$ by $\mathrm{TA}^{*}(X)$. It turns out that $\mathrm{TA}^{*}(X)$ for $X$ belonging to some class $\Gamma$ of separable metric spaces can naturally be considered as the strengthening of the standard perfect-set property of $\Gamma$ (that is, every uncountable $X \in \Gamma$ contains a perfect set). The following results shows that there is indeed a quite close relationship between this new perfect-set property and the classical one.

\footnotetext{
${ }^{41}$ We say that an ideal $\mathcal{I}$ has the weak diagonal sequence property of for every sequence $\left\{a_{n}\right.$ : $n<\omega\} \subseteq \mathcal{I} \backslash$ Fin there is $b \in \mathcal{I}$ such that $b \cap a_{n} \neq \emptyset$ for infinitely many $n$ 's.

${ }^{42}$ The reader is indeed invited to reproduce the simple direct proof of Proposition 18.2 or rely on Shoenfield's absoluteness and the fact that the second alternative of $\mathrm{TA}(X)$ can be forced if the first fails (see Proposition 18.8 below). More proofs of this result can be found in 37.
} 
Theorem 18.6 ([37]). The following statements are equivalent:

(1) Every uncountable co-analytic set of reals contains a perfect set.

(2) $\mathrm{TA}^{*}(X)$ holds for every co-analytic set of reals.

(3) For every real $r, L[r]$ fails to correctly compute the first uncountable ordinal.

Remark 18.7. Recall that the equivalence of (1) and (3) is a classical result of Solovay [96].

The following fact (due to the author and appearing in 37]) gives us an explanation of the close relationship between the one-dimensional and two-dimensional perfect-set properties, in view of the fact that we can always go to a forcing extension of the universe satisfying TA and $\mathfrak{p}>\omega_{1}$.

Proposition 18.8. Assume $\mathfrak{p}>\aleph_{1}$. Suppose $\mathcal{G}=(X, R)$ is an open graph on a Polish space $X$ and that $Y$ is an arbitrary subset of $X$. The following are equivalent:

(1) There is an uncountable $\mathcal{G}$-complete subset $Z \subseteq Y$,

(2) There is a $\mathcal{G}$-complete $G_{\delta}$-set $G$ such that $G \cap Y$ is uncountable.

Proof. To prove the nontrivial implication, we may assume that $Z$ is $\aleph_{1}$-dense in $X$. Fix a countable basis $\mathcal{B}$ of $X$. Consider the set $\mathcal{P}$ of all mappings $p: Z_{p} \rightarrow \mathcal{B}$ where $Z_{p}$ is a finite subset of $Z$ such that $x \in p(x)$ for all $x \in Z_{p}$ and

$$
\left(\forall x \neq y \in Z_{p}\right) p(x) \cap p(y)=\emptyset \text { and } p(x) \times p(y) \subseteq R .
$$

We order $\mathcal{P}$ by letting $p \leq q$ whenever $Z_{p} \supseteq Z_{q}$ and the family $p^{\prime \prime} Z_{p}$ refines the family $q^{\prime \prime} Z_{q}$. Then $\mathcal{P}$ is a $\sigma$-centered poset, so our assumption $\mathfrak{p}>\aleph_{1}$ gives us a filter $\mathcal{F}$ of $\mathcal{P}$ such that $\bigcup_{p \in \mathcal{F}} Z_{p}$ is uncountable and such that if we let $G=$ $\bigcap_{p \in \mathcal{F}} \bigcup p^{\prime \prime} Z_{p}$ then $G$ is the desired $\mathcal{G}$-complete $G$-delta set whose intersection with $Z$ is uncountable.

Remark 18.9. This also shows (using the same assumption) that whenever a sufficiently rich point-class $\Gamma$ has the Perfect-Set Property then TA $(\Gamma)$ implies TA* $(\Gamma)$.

We finish this section with a typical application of $\mathrm{TA}^{*}$.

Theorem 18.10 ([120]). If $X$ is a Polish space and if $K$ is any compact space then the family of all homeomorphic copies of $K$ inside $X$ is $\sigma$-linked ${ }^{43}$ unless the product $K \times 2^{\mathbb{N}}$ embeds into $X$.

\section{Chromatic Theory of Closed Graphs on Polish Spaces}

In this section we show the difference between the classes of open and closed graphs on separable metric spaces.

Example 19.1 ([113]). [The closed graph $\mathcal{G}_{c}=\left(\mathbb{N}^{\mathbb{N}}, R_{c}\right)$ ] To define $R_{c}$ we first associate to every $f \in \mathbb{N}^{\mathbb{N}}$ a sequence $\left\{f_{i}\right\}$ as follows. Let $n_{0}<n_{1}<\cdots$ be the list of all $n$ such that $f(2 n+1) \neq 0$. For a given $i$ let $f_{i}$ be defined by

$$
f_{i}\left\lceil n_{k}=f\left\lceil n_{k} \text { and } f_{i}\left(n_{k}+j\right)=f\left(2^{i}(2 j+1)\right)\right.\right.
$$

for $j<\omega$, where $k=\min \left\{\ell: f\left(2 n_{0}+1\right)+\cdots+f\left(2 n_{\ell}+1\right)>i\right\}$; let $f_{i}=f$ whenever such $k$ does not exist. Finally, define

$$
(f, g) \in R_{c} \Longleftrightarrow(\exists i)\left[f=g_{i} \text { or } g=f_{i}\right] .
$$

\footnotetext{
${ }^{43}$ Recall that a family $\mathcal{F}$ of nonempty sets is $\sigma$-linked if there is a countable decomposition $\mathcal{F}=\bigcup_{n<\omega} \mathcal{F}_{n}$ such that $(\forall n<\omega)\left(\forall F, G \in \mathcal{F}_{n}\right) F \cap G \neq \emptyset$.
} 
Claim 1. $R_{c} \cup \triangle$ is a closed symmetric relation on $\mathbb{N}^{\mathbb{N}}$.

Claim 2. $\mathcal{K}_{\aleph_{1}} \not \leq \mathcal{G}_{c}=\left(\mathbb{N}^{\mathbb{N}}, R_{c}\right)$.

Claim 3. $\operatorname{Chr}\left(\mathcal{G}_{c}\right)=\aleph_{1}$.

The following fact will give us Theorem 11.4 showing that in the context of forcing axiom the compactness number $\kappa_{\mathrm{C}}$ has only the trivial realization $\kappa_{\mathrm{C}}=\mathfrak{c}^{+}$.

Proposition 19.2 ([108]). Under $\mathrm{MA}_{\aleph_{1}}$, every subgraph of the graph $\mathcal{G}_{c}$ of size at most $\aleph_{1}$ is countably chromatic.

On the other hand the following result shows that there are natural conditions under which $\mathcal{G}$ does have subgraphs of size $\aleph_{1}$ that are not countably chromatic.

Proposition 19.3 ([115]). If $\mathfrak{b}=\aleph_{1}$ there is $X \subseteq \mathbb{N}^{\mathbb{N}}$ of size $\aleph_{1}$ such that the restriction $\mathcal{G}_{c} \uparrow X$ is not countably chromatic and in fact contains no uncountable $\mathcal{G}_{c}$-complete set.

Question 19.4. Is $\mathfrak{b}>\aleph_{1}$ equivalent to the statement that all subgraphs of $\mathcal{G}_{c}$ of size $\aleph_{1}$ are countably chromatic?

The example $\mathcal{G}_{c}=\left(\mathbb{N}^{\mathbb{N}}, R_{c}\right)$ is a closed graph on a Polish space which leads us to the following natural question.

Question 19.5. Is the set of cardinals

$$
\{1,2,3, \ldots\} \cup\left\{\aleph_{0}, \aleph_{1}, \mathfrak{c}\right\}
$$

equal to the chromatic number spectrum of closed graphs on Polish spaces?

It is known that the positive answer to this question is consistent so the question is really about a possibility of resolving this question using no additional axioms. To explain this recall that a graph $\mathcal{G}=(X, E)$ satisfies the countable chain condition if the poset of finite $\mathcal{G}$-independent subsets of $X$ satisfies the countable chain condition, or in other words if any uncountable family $\mathcal{F}$ of finite $\mathcal{G}$-independent subsets of $X$ contains two distinct sets $p$ and $q$ whose union $p \cup q$ is also $\mathcal{G}$-independent. The following dichotomy result shows that among closed graphs on Polish spaces there are many examples of graphs satisfying the countable chain condition.

Theorem 19.6 (113]). For every closed graph $\mathcal{G}=(X, E)$ on a Polish space $X$ either,

(1) $\mathcal{G}$ satisfies the countable chain condition, or

(2) $\mathcal{K}_{2^{\mathrm{N}}} \leq_{c} \mathcal{G}$.

Proof. Assume $\mathcal{K}_{2^{\mathrm{N}}} \mathbb{Z}_{c} \mathcal{G}$ and, towards showing that $\mathcal{G}$ satisfies the countable chain condition, let $\mathcal{F}$ be an uncountable family of finite $\mathcal{G}$-independent subsets of $X$. Applying a $\Delta$-system argument one sees that we may assume that the elements of $\mathcal{F}$ are pairwise disjoint and of some fixed cardinality $n$. Moreover, we may assume that for a fixed sequence $B_{0}, \ldots, B_{n-1}$ of open sets with pairwise disjoint closures we have that every element of $\mathcal{F}$ meets every $B_{i}$ in exactly one point and that

$$
(\forall i \neq j<n)\left(B_{i} \times B_{j}\right) \cap E=\emptyset .
$$

Thus $\mathcal{F}$ can be naturally identified with a subset of the product $\prod_{i<n} B_{i}$. Let $Y$ be the closure of $\mathcal{F}$ in this product. If there exist $x, y \in Y$ such that

$$
(\forall i<n)\left[x_{i} \neq y_{i} \text { and }\left(x_{i}, y_{i}\right) \notin E\right],
$$


using the fact that $\mathcal{F}$ is dense in $Y$, we would find $p \neq q$ in $\mathcal{F}$ such that $p \cup q$ is $\mathcal{G}$-discrete, as required. So, we assume that such $x$ and $y$ cannot be found in $Y$, and work for a contradiction. Choose a perfect subset $P$ of $Y$ such that

$$
(\forall x \neq y \in P)(\forall i<n) x_{i} \neq y_{i} .
$$

Thus, for every $x \neq y$ in $P$ there is $i<n$ such that $\left(x_{i}, y_{i}\right) \in E$. Applying Galvin's perfect-set Ramsey theorem (see [13]), we find a perfect $Q \subseteq P$ and $i<n$ such that

$$
(\forall x \neq y \in P)\left(x_{i}, y_{i}\right) \in E .
$$

It follows that the projection of $Q$ on the $i$ th coordinate is a perfect $\mathcal{G}$-complete subset of $X$, a contradiction.

Recall also the following statement considered above in Section 9

Definition 19.7 ([112]). [The Principle $\left.\Sigma_{B}^{2}\right] \operatorname{Chr}(\mathcal{G}) \leq \aleph_{1}$ for every Borel graph $\mathcal{G}$ satisfying the countable chain condition.

It has been shown in [112] that $\Sigma_{B}^{2}$ is consistent with the continuum large though it is still open whether the unrestricted version $\Sigma^{2}$ equivalent to $\mathrm{CH}$ ? Returning back to our problem of chromatic spectra of closed graphs on Polish spaces, we have the following result (an immediate consequence of Theorem 19.6) that shows that the minimal value of the chromatic spectrum is at least consistent with the usual axioms of set theory.

Theorem 19.8 ([113]). Assuming $\Sigma_{B}^{2}$, for every closed graph $\mathcal{G}=(X, E)$ on a Polish space $X$, either

(1) $\operatorname{Chr}(\mathcal{G}) \leq \aleph_{1}$, or

(2) $\mathcal{K}_{2^{\mathrm{N}}} \leq_{c} \mathcal{G}$.

Corollary 19.9. $\Sigma_{B}^{2}$ implies that the chromatic number $\operatorname{Chr}(\mathcal{G})$ of a closed graph $\mathcal{G}=(X, E)$ on a Polish space $X$ must take one of the following possible values $1,2,3, \ldots, \aleph_{0}, \aleph_{1}, \mathfrak{c}$.

What we were really showing so far is the extent to which the complete graph $\mathcal{G}_{2^{\mathrm{N}}}$ is a critical object in the class of all closed graphs on Polish spaces. We now give another similar example of a closed graph which itself is a critical object in this class though relative to the Borel chromatic theory rather that the ordinary chromatic theory of graphs.

Example $19.10(64)$. [The Closed Acyclic Graph $\left.\mathcal{G}_{0}=\left(2^{\mathbb{N}}, R_{0}\right)\right]$ Choose a sequence $t_{n} \in 2^{n} \quad(n<\omega)$ such that $\left(\forall t \in 2^{<\omega}\right)(\exists n) t \sqsubseteq t_{n}$. For $x \in 2^{\omega}$ we now define $\left(x_{i}\right)_{i<\omega} \subseteq 2^{\omega}$ as follows. Let $n_{0}<n_{1}<n_{2}<\cdots$ be the list of all $n$ such that $x\left\lceil n=t_{n}\right.$. Let

$$
\begin{aligned}
x_{i}\left\lceil n_{i}\right. & =x\left\lceil n_{i}\right. \\
x_{i}\left(n_{i}\right) & =1-x\left(n_{i}\right) \\
x_{i}(n) & =x(n) \text { for } n>n_{i}
\end{aligned}
$$

whenever $n_{i}$ exists; otherwise let $x_{i}=x$. Finally put

$$
(x, y) \in R_{0} \Longleftrightarrow(\exists i)\left[x=y_{i} \text { or } y=x_{i}\right] .
$$

Claim 1. $\mathcal{G}_{0}=\left(2^{\mathbb{N}}, R_{0}\right)$ is a closed acyclic graph and therefore $\operatorname{Chr}\left(\mathcal{G}_{0}\right)=2$. 
However, the corresponding Borel chromatic number and the corresponding Baire chromatic number of $\mathcal{G}_{0}$ behave quite differently.

Claim 2. $\operatorname{Chr}_{B}\left(\mathcal{G}_{0}\right)>\aleph_{0}$, or in other words, for every countable Borel partition, and in fact, for every countable Baire partition,

$$
2^{\mathbb{N}}=\bigcup_{i<\omega} B_{i}
$$

there exists $i<\omega$ and $x \neq y$ in $B_{i}$ such that $(x, y) \in R_{0}$.

The following result shows that $\mathcal{G}_{0}$ is the minimal Borel graph of uncountable Borel chromatic number in a similar way the complete graph $\mathcal{K}_{\aleph_{1}}$ is minimal in the class of open uncountably chromatic graphs on separable metric spaces.

Theorem 19.11 (64). Let $\mathcal{G}=(X, R)$ be an analytic graph ${ }^{44}$ on a Polish space $X$. Then exactly one of the following holds:

(1) $\operatorname{Chr}_{B}(\mathcal{G}) \leq \aleph_{0}$,

(2) $\mathcal{G}_{0} \leq_{c} \mathcal{G}$. ${ }^{45}$

Corollary 19.12 ([93]). A co-analytic equivalence relation $E$ defined on a Polish space $X$ has either countably many classes or a perfect set of pairwise inequivalent elements.

Proof. Apply Theorem 19.11 to the analytic graph $\mathcal{G}=\left(X, X^{2} \backslash E\right)$.

We finish this section with a remark that many new applications and variations of the $\mathcal{G}_{0}$-dichotomy can be found in the recent article [74].

\section{Part 3. Ideals of Countable Sets}

\section{Two Dual Dichotomies for Ideals of Countable Sets}

In this section we introduce two dichotomies for ideals of countable subsets of some index set $S$. There are two conditions that one needs to put on such ideals in order to obtain consistent dichotomies.

Definition 20.1. Suppose $\mathcal{I} \subseteq[S] \leq \aleph_{0}$ is an ideal ${ }^{46} \mathcal{I}$ is a $P$-ideal if for every sequence $\left\{a_{n}: n<\omega\right\} \subseteq \mathcal{I}$ there exists $b \in \mathcal{I}$ such that $a_{n} \subseteq^{*} b{ }^{47}$ for all $n<\omega$.

Example 20.2. The ideal $\mathcal{I}=[S]^{\leq \aleph_{0}}$ of all countable subsets of some set $S$ is a P-ideal.

Example 20.3. For $\mathcal{C} \subseteq \mathcal{P}(S)$ countable (or, more generally, for $\mathcal{C} \subseteq \mathcal{P}(S)$ of size $<\mathfrak{b})$, the orthogonal

$$
\mathcal{I}=\mathcal{C}^{\perp}=\left\{a \in[S]^{\leq \aleph_{0}}:(\forall C \in \mathcal{C}) a \cap C \in \operatorname{Fin}\right\}
$$

is a P-ideal.

So the larger cardinal $\mathfrak{b}$ is, the larger is the class of P-ideals, and therefore stronger are the dichotomies that we would like to associate to this class of ideals. The following simple fact is showing us how the operation of taking the orthogonal transfers the dichotomies that one usually would like to consider in this context.

\footnotetext{
$44_{\text {that is, }} R \subseteq X^{2}$ is analytic

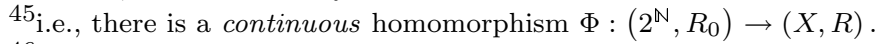

${ }^{46} \mathrm{~A}$ collection of subsets of $S$ closed under taking unions and subsets and $\{\{x\}: x \in S\} \subseteq \mathcal{I}$.

${ }^{47}$ Recall that $a_{n} \subseteq{ }^{*} b$ means that $a_{n} \backslash b \in$ Fin.
} 
Proposition 20.4. Let $\mathcal{J}$ be $a<\mathfrak{p}$-generated ideal of countable subsets of some set $S$ and let $\mathcal{I}=\mathcal{J}^{\perp}$ be its orthogonal. Then the following statements are equivalent:

(A) The ideal $\mathcal{J}$ has one of the following two properties:

(1) There is uncountable $X \subseteq S$ such that $X \cap b \in$ Fin for all $b \in \mathcal{J}$.

(2) There is a decomposition $S=\bigcup_{n<\omega} S_{n}$ such that $\left[S_{n}\right]^{\aleph_{0}} \subseteq \mathcal{J}$ for all $n<\omega$.

(B) The ideal $\mathcal{I}$ has one of the following two properties:

(1) There is uncountable $X \subseteq S$ such that $[X]^{\aleph_{0}} \subseteq \mathcal{I}$.

(2) There is a decomposition $S=\bigcup_{n<\omega} S_{n}$ such that $S_{n} \cap a \in$ Fin for all $n<\omega$ and $a \in \mathcal{I}$.

The dichotomy (A) appears in [106] in notation $(*)$ as the combinatorial essence of several problems solved by the method of taking elementary submodels as side conditions when building proper posets (see [102, [104, 108]).

Theorem 20.5 ([106]). Assume PFA. Then for every $\aleph_{1}$-generated ideal $\mathcal{J}$ of countable subsets of some set $S$, either

(1) there is uncountable $X \subseteq S$ such that $X \cap b \in$ Fin for all $b \in \mathcal{J}$, or

(2) there is a decomposition $S=\bigcup_{n<\omega} S_{n}$ such that $\left[S_{n}\right]^{\aleph_{0}} \subseteq \mathcal{J}$ for all $n<\omega$.

The proof consists in showing that if (2) fails then there is a proper poset that forces the uncountable set satisfying (1). Looking back at the Proposition 20.4. we see that a proper poset would force alternative $\mathrm{B}(1)$ whenever $\mathrm{B}(2)$ fails for every P-ideal $\mathcal{I}$ (not just one of the form $\mathcal{I}=\mathcal{J}^{\perp}$, That the proof of Theorem 20.5 gives the second dichotomy (B) with no restriction on the ideal $\mathcal{I}$ (except that it is a P-ideal) was realized by the author while solving specific problems about coherent sequences on $\omega_{1}$ (see, for example, [102], Theorem 6, 108, Theorem 8.13, [115, Lemma 1 and Lemma $1^{*}$, and [30]) asked by Galvin [43, Dow [25, Leiderman and Malykhin [68, and Watson (Problem 174 from [75, ${ }^{49}$ ).

Theorem 20.6 ([102,, 108, , 115, [30]). Assume PFA. Then for every P-ideal $\mathcal{I}$ of countable subsets of some set $S$, either

(1) there is uncountable $X \subseteq S$ such that $[X]^{\aleph_{0}} \subseteq \mathcal{I}$, or

(2) there is a decomposition $S=\bigcup_{n<\omega} S_{n}$ such that $S_{n} \cap a \in$ Fin for all $n<\omega$ and $a \in \mathcal{I}$

[Sketch of Proof] Fix a P-ideal $\mathcal{I}$ of countable subsets of $S$ and assume that the second alternative fails, or in other words, $S$ cannot be decomposed into countably many sets orthogonal to $\mathcal{I}$. We shall find a proper poset $\mathcal{P}$ which forces an uncountable set $X \subseteq S$ such that $[X]^{\aleph_{0}} \subseteq \mathcal{I}$.

Fix a sufficiently large regular cardinal $\theta$ such that the structure $\left(H_{\theta}, \in\right)$ contains the set $[S]^{\aleph_{0}}$. For a countable elementary submodel $N \prec H_{\theta}$, we fix $b_{N} \in \mathcal{I}$ such that $b_{N} \subseteq S \cap N$ and

$$
(\forall a \in \mathcal{I} \cap N) a \subseteq^{*} b_{N},
$$

and we fix a point $x_{N} \in S$ such that

$$
(\forall X \in \mathcal{P}(S) \cap N)\left[X \perp \mathcal{I} \Rightarrow x_{N} \notin X\right] .
$$

\footnotetext{
${ }^{48}$ Note that by Example 20.3 the ideal $\mathcal{I}$ of Proposition 20.4 is indeed a P-ideal.

${ }^{49}$ The problem was motivated by the well known problem of the existence of Ostaszewski space on the basis of $\mathrm{CH}$ rather than $\diamond$.
} 
Expanding $\left(H_{\theta}, \in\right)$ by adding a well-ordering $<_{w}$ of $H_{\theta}$ we may assume $N \mapsto b_{N}$ and $N \mapsto x_{N}$ are definable maps.

Let $\mathcal{P}$ be the collection of all finite $\in$-chains $p$ of countable elementary submodels of $\left(H_{\theta}, \in,<_{w}\right)$. For $p, q \in \mathcal{P}$, set $p \leq q$ if and only if,

(a) $p \supseteq q$, and

(b) $(\forall M \in q)(\forall N \in M \cap(p \backslash q))\left[x_{N} \in b_{M}\right]$.

Claim 1. $\mathcal{P}$ is a proper poset.

Proof Sketch. Consider a sufficiently large regular cardinal $\kappa$ and a countable elementary submodel $M \prec\left(H_{\kappa}, \in\right)$ containing $\mathcal{P}$, and let $p \in \mathcal{P} \cap M$ be arbitrarily chosen. Let

$$
q=p \cup\left\{M \cap H_{\theta}\right\} .
$$

We shall show that $q$ is $(M, \mathcal{P})$-generic. Consider dense-open $\mathcal{D} \subseteq \mathcal{P}, \mathcal{D} \in M$, and $r \leq q$. We may assume $r \in \mathcal{D}$. For $s \in \mathcal{D}$ end-extending $r \cap M$, let

$$
x^{s}=\left\langle x_{1}^{s}, x_{2}^{s}, \ldots, x_{l}^{s}\right\rangle
$$

where

$$
\left\{x_{N}: N \in s \backslash(r \cap M)\right\}=\left\{x_{1}^{s}, x_{2}^{s}, \ldots, x_{l}^{s}\right\}
$$

enumerated according to the $\in$-ordering of $s$. Let $k$ be the length of the sequence $x^{r}$ associated to the condition $r$ which clearly end-extends $r \cap M$. Let

$$
\mathcal{F}=\left\{x \in S^{k}:(\exists s \sqsupseteq r \cap M)\left[s \in \mathcal{D} \quad \& \quad x_{s}=x\right]\right\} .
$$

Then $\mathcal{F} \in M$ and $x^{r}=\left\langle x_{N_{1}}, x_{N_{2}}, \ldots, x_{N_{k}}\right\rangle \in \mathcal{F}$. It follows that $\mathcal{F}$ is Namba relative to the co-ideal

$$
\mathcal{H}=\{X \subseteq S: \mathcal{I} \uparrow X \not \models \text { alternative }(2)\} .
$$

So in particular $\mathcal{F}$ contains a subfamily $\mathcal{F}_{0} \in M$ that forms the set of maximal nodes of an everywhere $\mathcal{H}$-branching subtree of $S \leq k$ of height $k$. Let

$$
X_{1}=\left\{\xi \in S:\left(\exists x \in \mathcal{F}_{0}\right) \xi=x_{1}\right\} .
$$

Then $X_{1} \in M \cap \mathcal{H}$. So there is infinite $a_{1} \in \mathcal{I} \cap M$ such that $a_{1} \subseteq X_{1}$. Then $a_{1} \subseteq^{*} b_{N_{i}}$ for all $i=1,2, \ldots, k$. So we can pick $\xi_{1} \in a_{1}$ such that $\xi_{1} \in \bigcap_{i=1}^{k} b_{N_{i}}$. Let

$$
X_{2}=\left\{\xi \in S:\left(\exists x \in \mathcal{F}_{0}\right)\left[\xi_{1}=x_{1} \quad \& \quad \xi=x_{2}\right]\right\} .
$$

Then $X_{2} \in M \cap \mathcal{H}$. So there must be infinite $a_{2} \in \mathcal{I} \cap M$ such that $a_{2} \subseteq X_{2}$. Then again $a_{2} \subseteq^{*} \bigcap_{i=1}^{k} b_{N_{i}}$, so we can choose $\xi_{2} \in a_{2} \cap \bigcap_{i=1}^{k} b_{N_{i}}$, and so on. At the end of this process one obtains $\left\{\xi_{1}, \xi_{2}, \ldots, \xi_{k}\right\} \subseteq \bigcap_{i=1}^{k} b_{N_{i}}$ such that

$$
\left\langle\xi_{1}, \xi_{2}, \ldots, \xi_{k}\right\rangle \in \mathcal{F} \cap M
$$

Pick $\bar{r} \in \mathcal{D} \cap M$ end-extending $r \cap M$ such that $x_{\bar{r}}=\left\langle\xi_{1}, \xi_{2}, \ldots, \xi_{k}\right\rangle$. Then $r$ and $\bar{r}$ are two compatible conditions of $\mathcal{P}$ and, in fact, $r \cup \bar{r}$ is their common extension.

It turns out that the second dichotomy (B) is a statement with an interest independent from PFA, so let us give it a special name.

Definition 20.7. [P-Ideal Dichotomy] For every P-ideal $\mathcal{I}$ of countable subsets of some set $S$, either

(1) there is uncountable $X \subseteq S$ such that $[X]^{\aleph_{0}} \subseteq \mathcal{I}$, or

(2) $S$ can be decomposed into countably many sets orthogonal to $\mathcal{I}$. 
The following result is only one of the facts which points out the special character of the second dichotomy.

Theorem 20.8 ( $\left.[124]^{50}\right)$. The P-ideal dichotomy is consistent with the GCH relative to the consistency of the existence of a supercompact cardinal.

\section{PID And the HausdorfF Condition on Gaps}

Fix an infinite set $S$ and for each countable subset $a$ of $S$ fix a well ordering $<_{a}$ of $a$ of order type $\leq \omega$. Then for $n<\omega$, we can let $a[n]$ denote the set formed by taking the first $n$ elements of $a$ according to $<_{a}$. If $S$ itself is countable, it is more convenient to fix its enumeration $S=\left\{s_{k}: k<\omega\right\}$ and for $a \subseteq S$ and $n<\omega$, let $a[n]=a \cap\left\{s_{k}: k<n\right\}$. This will give us a way to state the Hausdorff original condition on gaps in the context of $\mathcal{P}(S) /$ Fin rather than just $\mathcal{P}(\mathbb{N}) /$ Fin.

Definition 21.1. A sequence $\left\{\left(a_{\alpha}, b_{\alpha}\right): \alpha<\omega_{1}\right\}$ of pairs of countable subsets of $S$ satisfies the Hausdorff condition if

$$
\begin{gathered}
b_{\alpha} \subseteq^{*} b_{\beta} \text { whenever } \alpha<\beta<\omega_{1}, \\
a_{\alpha} \cap b_{\beta} \in \text { Fin for all } \alpha, \beta<\omega_{1} \text {, and } \\
\left\{\alpha<\beta: a_{\alpha} \cap b_{\beta} \subseteq a_{\alpha}[n]\right\} \in \text { Fin for all } \beta<\omega_{1} \text { and } n<\omega
\end{gathered}
$$

Remark 21.2. In Hausdorff's original definition, the set $S$ was countable and the sequence $a_{\alpha}\left(\alpha<\omega_{1}\right)$ was also assumed to be increasing under $\subseteq^{*}$. Note also that this notion does not depend on the choice of well-orderings $<_{a}\left(a \in[S]^{\leq \aleph_{0}}\right)$.

First in order is a lemma which shows that a sequence satisfying the Hausdorff condition is indeed a gap in a quite strong and absolute sense.

Lemma 21.3. Suppose that a sequence $\left\{\left(a_{\xi}, b_{\xi}\right): \xi<\omega_{1}\right\}$ of pairs of countably infinite subsets of some set $S$ satisfies the Hausdorff condition. Then $\left\{a_{\xi}: \xi<\omega_{1}\right\}$ is not countably generated in the orthogonal of $\left\{b_{\xi}: \xi<\omega_{1}\right\}$.

Proof. Otherwise we can find a single subset $C$ of $S$ and an uncountable subset $\Gamma$ of $\omega_{1}$ such that $a_{\gamma} \subseteq^{*} C$ and $b_{\gamma} \cap C \in$ Fin for all $\gamma \in \Gamma$. We may assume that there is some integer $n$ such that

$$
a_{\alpha} \backslash C \subseteq a_{\alpha}[n] \text { for all } \alpha \in \Gamma .
$$

Moreover, increasing $n$ and shrinking $\Gamma$, we may assume that the sequence of finite sets $b_{\beta}^{*}=b_{\beta} \cap C(\beta \in \Gamma)$ forms a $\Delta$-system with root $r$ such that

$$
\begin{gathered}
a_{\alpha} \cap r \subseteq a_{\alpha}[n] \text { for all } \alpha \in \Gamma, \text { and } \\
a_{\alpha} \cap\left(b_{\beta}^{*} \backslash r\right)=\emptyset \text { for all } \alpha, \beta \in \Gamma \text { with } \alpha<\beta .
\end{gathered}
$$

Choose $\beta \in \Gamma$ such that $\Gamma_{0}=\Gamma \cap \beta$ is infinite. Then $a_{\alpha} \cap b_{\beta} \subseteq a_{\alpha}[n]$ for all $\alpha \in \Gamma_{0}$ contradicting the Hausdorff condition.

Fix two orthogonal families $\mathcal{A}$ and $\mathcal{B}$ of countable subsets of $S$. Let

$$
\mathcal{I}_{(\mathcal{A}, \mathcal{B})}=\left\{A \in[\mathcal{A}]^{\leq \aleph_{0}}:(\exists b \in \mathcal{B})(\forall n<\omega)\{a \in A: a \cap b \subseteq a[n]\} \in \text { Fin. }\right\}
$$

\footnotetext{
${ }^{50}$ The special case $S=\omega_{1}$ of this result appears in [2] and it does not require large cardinals. However, as we are going to see below, even the case $S=\omega_{2}$ has some large cardinal strength.
} 
Lemma 21.4. $\mathcal{I}_{(\mathcal{A}, \mathcal{B})}$ is a P-ideal of countable subsets of $\mathcal{A}$ whenever $\mathcal{B}$ is a $P$-ideal of countable subsets of $S$ and either $S$ is countable or $\mathcal{A}$ consists of pairwise disjoint subsets of $S$.

Proof. We only treat the assumption that $\mathcal{A}$ consists of pairwise disjoint sets since the treatment of the other assumption is quite analogous and simpler. So let $\left\{A_{k}: k<\omega\right\} \subseteq \mathcal{I}_{(\mathcal{A}, \mathcal{B})}$ be a given sequence and for each $k$, let $b_{k} \in \mathcal{B}$ witness $A_{k} \in \mathcal{I}_{(\mathcal{A}, \mathcal{B})}$. Pick $b \in \mathcal{B}$ such that $b_{k} \subseteq^{*} b$ for all $k<\omega$. Note that for each $n, k<\omega$, the set

is finite, since

$$
A_{k}(n)=\left\{a \in B_{k}: a \cap b \subseteq a[n]\right\}
$$

$$
A_{k}(n) \subseteq\left\{a \in A_{k}: a \cap b_{k} \subseteq a[n]\right\} \cup\left\{a \in A_{k}: a \cap\left(b_{k} \backslash b\right) \neq \emptyset\right\} .
$$

Let $A=\bigcup_{n<\omega}\left(A_{n} \backslash A_{n}(n)\right)$. Then $b$ is a witness of $A \in \mathcal{I}_{(\mathcal{A}, \mathcal{B})}$. Moreover, $A_{n} \subseteq^{*} A$ for all $n<\omega$, as required.

Lemma 21.5. Suppose that $\mathcal{A}$ and and $\mathcal{B}$ are two orthogonal ideals of countable subsets of some set $S$ and that $\mathcal{B}$ is a P-ideal. If there is uncountable $\mathcal{X} \subseteq \mathcal{A}$ such that $[\mathcal{X}]^{\aleph_{0}} \subseteq \mathcal{I}_{(\mathcal{A}, \mathcal{B})}$ then there is a sequence $\left\{\left(a_{\xi}, b_{\xi}\right): \xi<\omega_{1}\right\} \subseteq \mathcal{A} \times \mathcal{B}$ satisfying the Hausdorff condition.

Proof. Choose a one-to one sequence $\left\{a_{\alpha}: \alpha<\omega_{1}\right\} \subseteq \mathcal{X}$. Then for every $\beta<\omega_{1}$, we can chose $b_{\beta} \in \mathcal{B}$ witnessing that $\left\{a_{\alpha}: \alpha<\beta\right\} \in \mathcal{I}_{(\mathcal{A}, \mathcal{B})}$. During the course of the proof of Lemma 21.4 we have seen that any set which almost includes $b_{\beta}$ will still witness this, so we may further assume that $b_{\beta} \subseteq^{*} b_{\gamma}$ whenever $\beta<\gamma<\omega_{1}$. Then $\left\{\left(a_{\alpha}, b_{\alpha}\right): \alpha<\omega_{1}\right\}$ satisfies the Hausdorff condition.

Lemma 21.6. Suppose that $\mathcal{A}$ and $\mathcal{B}$ are two orthogonal ideals of countable subsets of some set $S$ and that $\mathcal{B}$ is a P-ideal. If $\mathcal{A}$ can be covered by countably many sets $\mathcal{A}_{n}(n<\omega)$ orthogonal to $\mathcal{I}_{(\mathcal{A}, \mathcal{B})}$, then $\mathcal{A}$ is countably generated in $\mathcal{B}^{\perp}$.

Proof. If $S$ is countable, take $\mathcal{C}=\left\{\bigcup \mathcal{A}_{n}: n<\omega\right\}$. Then $\mathcal{C} \subseteq \mathcal{B}^{\perp}$ and every element of $\mathcal{A}$ is almost included in some set from $\mathcal{C}$. Assume now that $\mathcal{A}$ consists of pairwise disjoint sets. Fix $n<\omega$. Then for every $b \in \mathcal{B}$ there is $k(b, n)<\omega$ such that

$$
a \cap b \subseteq a[k(b, n)] \text { for all } a \in \mathcal{A}_{k} .
$$

Since $\mathcal{B}$ is $\sigma$-directed under $\subseteq^{*}$ there is $k(n)<\omega$ such that $\{b \in \mathcal{B}: k(b, n)=k(n)\}$ is $\subseteq^{*}$-cofinal in $\mathcal{B}$. Let $\mathcal{C}=\left\{\bigcup\left\{a \backslash a[k(n)]: a \in \mathcal{A}_{n}\right\}: n<\omega\right\}$. Then $\mathcal{C}$ is a countable family of subsets of $S$ that are orthogonal to $\mathcal{B}$ such that every element of $\mathcal{A}$ is almost included in some set from $\mathcal{C}$

Theorem 21.7 (124]). Assume PID. Let $\mathcal{A}$ and $\mathcal{B}$ be two orthogonal families of subsets of some countable set $S$ such that the family $\mathcal{B}$ is a P-ideal. Then either

(1) $\mathcal{A} \times \mathcal{B}$ contains a Hausdorff gap $\left\{\left(a_{\alpha}, b_{\alpha}\right): \alpha<\omega_{1}\right\}$, or

(2) $\mathcal{A}$ is countably generated in $\mathcal{B}^{\perp}$.

Corollary 21.8. Assuming PID, the orthogonal of any $P_{\aleph_{1}}$-ideal ${ }^{51}$ on $\omega$ is countably generated.

\footnotetext{
${ }^{51}$ Recall that an ideal $\mathcal{I}$ of countable subsets of some set is a $P_{\aleph_{1}}$-ideal if for every sequence $\left\{a_{\xi}: \xi<\omega_{1}\right\} \subseteq \mathcal{I}$ there is $b$ in $\mathcal{I}$ such that $a_{\xi} \backslash b$ is finite for all $\xi<\omega_{1}$.
} 
Corollary 21.9. PID implies $\mathfrak{b} \leq \aleph_{2}$.

Question 21.10. Does PID imply $\mathfrak{c} \leq \aleph_{2}$ ?

Theorem 21.11 ([108). Assume PID. Let $\mathcal{A}$ and $\mathcal{B}$ be two orthogonal families of countable subsets of some set $S$ such that the family $\mathcal{B}$ is a P-ideal whose orthogonal $\mathcal{B}^{\perp}$ is $\aleph_{1}$-generated. Then either

(1) $\mathcal{A} \times \mathcal{B}$ contains a Hausdorff gap, or

(2) $\mathcal{A}$ is countably generated in $\mathcal{B}^{\perp}$.

Proof. If there is countable $S_{0}$ such that $\mathcal{A} \uparrow S_{0}$ is not countably generated in the orthogonal of $\mathcal{B}\left\lceil S_{0}\right.$, we are done by Theorem 21.7. Otherwise, assuming that $\mathcal{A}$ is not countably generated in $\mathcal{B}^{\perp}$, and using the fact that $\mathcal{B}^{\perp}=\{X \subseteq S: X \perp \mathcal{B}\}$ is an $\aleph_{1}$-generated ideal in $\mathcal{P}(S) /$ Fin, we can find a subfamily $\mathcal{A}_{0}$ of $\mathcal{A}$ consisting of pairwise disjoint sets and still not countably generated in $\mathcal{B}^{\perp}$. Then using Lemmas 21.4 21.5 and 21.6. and PID, we get a Hausdorff gap $\left\{\left(a_{\alpha}, b_{\alpha}\right): \alpha<\omega_{1}\right\}$ in $\mathcal{A}_{0} \times \mathcal{B}$.

Corollary 21.12. Assume PFA. Let $\mathcal{A}$ and $\mathcal{B}$ be two orthogonal families of countable subsets of some set $S$ such that the family $\mathcal{B}$ is a P-ideal. Then either

(1) $\mathcal{A} \times \mathcal{B}$ contains a Hausdorff gap, or

(2) $\mathcal{A}$ is countably generated in $\mathcal{B}^{\perp}$.

Proof. This is really the generic version of Theorem 21.11 as it can be reduced to it by noticing that if $\mathcal{B}$ is a P-ideal of countable subsets of some set $S$ then the orthogonal $\mathcal{B}^{\perp}$ computed in any $\sigma$-closed forcing extension is generated by the orthogonal $\mathcal{B}^{\perp}$ computed in the ground model.

\section{PID and Coherent Sequences}

Definition 22.1. Two maps $f: a \rightarrow \omega$ and $g: b \rightarrow \omega$ are coherent if

$$
\{x \in a \cap b: f(x) \neq g(x)\} \in \text { Fin. }
$$

Definition 22.2. Two maps $f: a \rightarrow \omega$ and $g: b \rightarrow \omega$ are weakly coherent if

$$
(\forall c \subseteq a \cap b)[f\lceil c \text { is unbounded } \Longleftrightarrow g\lceil c \text { is unbounded }] .
$$

Theorem 22.3 ([124]). Assume PID. Then for every weakly coherent family

$$
f_{a}: a \rightarrow \omega \quad(a \in \mathcal{I})
$$

indexed by some $P$-ideal $\mathcal{I}$ on some set $S$, either

(1) there is uncountable $X \subseteq S$ such that $[X]^{\aleph_{0}} \subseteq \mathcal{I}$ and $f_{a} \uparrow X$ is finite-to-one for all $a \in \mathcal{I}$, or

(2) there is $g: S \rightarrow \omega$ which coheres weakly with all $f_{a}(a \in \mathcal{I})$.

Proof. Let

$$
\mathcal{I}_{0}=\left\{a \in \mathcal{I}:|a| \leq \aleph_{0} \quad \& \quad(\forall b \in \mathcal{I}) f_{b}\lceil a \text { is finite-to-one }\} .\right.
$$

Claim 1. $\mathcal{I}$ is a P-ideal.

Case 1: $[X]^{\aleph_{0}} \subseteq \mathcal{I}_{0}$ for some uncountable $X \subseteq S$. Then

$$
(\forall b \in \mathcal{I}) f_{b} \uparrow X \text { is finite-to-one. }
$$

Case 2: $S=\bigcup_{n<\omega} S_{n}$ such that $S_{n} \perp \mathcal{I}_{0}$ for all $n<\omega$. Pick $g: S \rightarrow \omega$ such that $(\forall n) g^{-1}(n) \subseteq S_{n}$. Then $g$ weakly coheres with every $f_{b} \quad(b \in \mathcal{I})$. 
Corollary 22.4. Assume PID. Then for every family

$$
f_{a}: a \rightarrow \omega \quad(a \in \mathcal{I})
$$

of weakly coherent functions indexed by a $P_{\aleph_{1}}$-ideal $\mathcal{I}$ on some set $S$ there is

$$
g: S \rightarrow \omega
$$

which weakly coheres with every $f_{a} \quad(a \in \mathcal{I})$.

Proof. Since $\mathcal{I}$ is a $P_{\aleph_{1}}$-ideal, the first alternative of the theorem is impossible.

Theorem 22.5 ([124]). PID implies that $\square(\kappa)$ fails for all $\kappa$ of cofinality $>\omega_{1}$.

Proof Sketch. We show using PID that no ordinal $\theta$ of cofinality $>\omega_{1}$ supports a nontrivial coherent $C$-sequence $C_{\alpha} \quad(\alpha<\theta)$.

Given a $C$-sequence $C_{\alpha} \quad(\alpha<\theta)$, define

$$
\rho_{2}:[\theta]^{2} \rightarrow \omega
$$

recursively by

$$
\rho_{2}(\alpha, \beta)=\rho_{2}\left(\alpha, \min \left(C_{\beta} \backslash \alpha\right)\right)+1,
$$

with the initial condition $\rho_{2}(\alpha, \alpha)=0$. We shall need the following two properties of $\rho_{2}$ (see [128]):

(1) If $C_{\alpha}(\alpha<\theta)$ is coherent then

$$
\sup _{\xi<\alpha}\left|\rho_{2}(\xi, \alpha)-\rho_{2}(\xi, \beta)\right|<\infty
$$

whenever $\alpha<\beta<\theta$.

(2) If $C_{\alpha}(\alpha<\theta)$ is coherent and nontrivial then

$$
(\forall n<\omega)(\forall A, B \subseteq \theta)\left[\sup A=\sup B=\theta \Rightarrow(\exists \alpha \in A)(\exists \beta \in B) \rho_{2}(\alpha, \beta)>n\right] .
$$

Property (1) gives that

$$
f_{\alpha}=\rho_{2}(\cdot, \alpha): \alpha \rightarrow \omega \quad(\alpha<\theta)
$$

is a family of weakly coherent mappings. Using PID, we have

$$
g: \theta \rightarrow \omega
$$

such that for every $\alpha<\theta$,

$$
(\forall X \subseteq \alpha)\left[f_{\alpha} \uparrow X \text { is unbounded } \Rightarrow g\lceil X \text { is unbounded }] .\right.
$$

This is impossible if $C_{\alpha}(\alpha<\theta)$ has property (2).

It follows that PID has considerable large cardinal strength and that a deep work about inner models of set theory and determinacy allows us to state the following kind of consequences.

Theorem 22.6 ([55]). The P-ideal dichotomy implies the projective determinacy.

Definition 22.7. For functions

$$
f: \alpha \rightarrow \omega \text { and } g: \beta \rightarrow \omega
$$

defined on ordinals $\alpha$ and $\beta$ respectively, set

(1) $f \leq_{l} g$ if $\alpha<\beta$ and

$$
(\forall X \subseteq \alpha)[f\lceil X \text { is unbounded } \Rightarrow g \uparrow X \text { is unbounded }] .
$$


(2) $f \leq_{r} g$ if $\alpha<\beta$ and

$(\forall X \subseteq \alpha)[g\lceil X$ is unbounded $\Rightarrow f \uparrow X$ is unbounded $]$.

Note that above we have shown the following:

Theorem 22.8 ([124]). Assume PID. Then for every ordinal $\theta$ of cofinality $>\omega_{1}$ and every sequence $f_{\alpha}: \alpha \rightarrow \omega \quad(\alpha<\theta)$ such that

$$
f_{\alpha} \leq_{l} f_{\beta} \text { whenever } \alpha<\beta,
$$

there is $g: \theta \rightarrow \omega$ such that

$$
f_{\alpha} \leq_{r} g \text { for all } \alpha<\theta .
$$

The following result shows that a similar fact holds for the ordering $\leq_{r}$ as well.

Theorem 22.9 ([135]). Assume PID. Then for every ordinal $\theta$ of cofinality $>\mathfrak{c}$ and every sequence $f_{\alpha}: \alpha \rightarrow \omega \quad(\alpha<\theta)$ such that

$$
f_{\alpha} \leq_{r} f_{\beta} \text { whenever } \alpha<\beta,
$$

there is $g: \theta \rightarrow \omega$ such that

$$
\left(\forall X \in[\theta]^{\aleph_{0}}\right)\left[g\left\lceil X \text { is bounded } \Rightarrow(\exists \alpha) f_{\alpha} \uparrow X \text { is bounded }\right] .\right.
$$

Proof. Let

$$
\mathcal{I}=\left\{a \in[\theta]^{\aleph_{0}}:(\forall \alpha<\theta) f_{\alpha}\lceil a \text { is finite-to-one }\} .\right.
$$

Claim 1. I is a P-ideal.

To see this, fix $\left\{a_{n}: n<\omega\right\} \subseteq \mathcal{I}$. If no $b \subseteq \bigcup_{n} a_{n}$ such that $b \supseteq^{*} a_{n}$ for all $n$ belongs to $\mathcal{I}$ then we can find $\beta<\theta$ such that for every such $b$ there is $\alpha<\beta$ such that $f_{\alpha}\left\lceil b\right.$ is not finite-to-one. Since $f_{\alpha} \leq_{l} f_{\beta}$ for all $\alpha<\beta$, it follows that $f_{\beta}\lceil b$ is also not finite-to-one, a contradiction.

Corollary 22.10 ([135]). PID implies $\theta^{\aleph_{0}}=\theta$ for all regular cardinals $\theta \geq \mathfrak{c}$.

We prove this by induction on regular cardinals $\theta \geq \mathfrak{c}$. The only problematic case is a cardinal $\theta$ of the form $\theta=\kappa^{+}$for some $\kappa$ of cofinality $\omega$. For this, fix a sequence $\left\{\kappa_{n}: n<\omega\right\}$ of regular cardinals converging to $\kappa$ and as before fix $\tau:[\theta]^{2} \rightarrow \omega$ such that

$$
\begin{gathered}
\tau(\alpha, \gamma) \leq \max \{\tau(\alpha, \beta), \tau(\beta, \gamma)\} \text { for } \alpha<\beta<\gamma<\theta \\
|\{\xi<\alpha: \tau(\xi, \alpha) \leq n\}| \leq \kappa_{n} \text { for all } \alpha<\theta \text { and } n<\omega .
\end{gathered}
$$

Let $f_{\alpha}:=\tau(\cdot, \alpha): \alpha \rightarrow \omega \quad(\alpha<\theta)$. Then $f_{\alpha} \leq_{r} f_{\beta}$ whenever $\alpha<\beta$. Let $g: \theta \rightarrow \omega$ be such that for every countable $X \subseteq \theta$ on which $g$ is bounded there is $\alpha<\theta$ such that $f_{\alpha}$ is bounded on $X$. It follows that

$$
\begin{gathered}
\theta^{\aleph_{0}}=\left|\bigcup_{n} g^{-1}[n]\right|=\mid\left\{X \in[\theta]^{\aleph_{0}}:(\exists \alpha>\sup X) f_{\alpha}\lceil X \text { is bounded }\}\right. \\
=\sum_{\alpha<\theta, n \in \omega}|\{\xi<\alpha: \tau(\xi, \alpha) \leq n\}|=\theta .
\end{gathered}
$$

Corollary 22.11 ([135]). PID implies SCH. 


\section{PID AND THE S-SPACE PROBLEM}

In this section we mention the original source of PID, a natural topological problem asking whether every hereditarily separable regular space is Lindelö. The importance of this problem was fully established by the early 1960's and it has been frequently referred as the $S$-space problem (see [87). The following example points immediately towards the nature of the problem and the fact that PID might be relevant.

Example 23.1 ([108). If $\mathfrak{b}=\omega_{1}$ then there is a regular first countable hereditarily separable space which is not Lindelöf. In fact the space is a subspace of $\mathbb{N}^{\mathbb{N}}$ where the usual separable metric topology is extended by making the sets of the form $\{x:(\forall n) x(n) \leq y(n)\},\left(y \in \mathbb{N}^{\mathbb{N}}\right)$ open.

Suppose $X$ is a non-Lindelöf subspace of some regular space $K$. This means that for each $x \in X$ we can choose an open neighborhood $U_{x}$ of $x$ in $K$ such that

$$
X \backslash \bigcup_{x \in X_{0}} U_{x} \neq \emptyset \text { for all countable } X_{0} \subseteq X \text {. }
$$

Since $K$ is regular, for each $x \in X$ we choose another open neighborhood $V_{x}$ of $x$ in $K$ such that $\overline{V_{x}} \subseteq U_{x}$. Let

$$
\mathcal{I}_{X}=\left\{A \in[X]^{\leq \aleph_{0}}:(\forall x \in X) A \cap \overline{V_{x}} \in \mathrm{FIN}\right\} \text {, }
$$

the ideal of countable subsets of $X$ that are orthogonal to $\left\{\overline{V_{x}}: x \in X\right\}$. If $\mathfrak{b}>|X|$, the ideal $\mathcal{I}_{X}$ is a P-ideal, so let us examine the two alternatives of PID. First of all note that any $Y \subseteq X$ with property $[Y]^{\aleph_{0}} \subseteq \mathcal{I}_{X}$ must be discrete. On the other hand if some subset $Y$ of $X$ is orthogonal to $\mathcal{I}_{X}$ then it cannot contain a countable subset $D$ such that

$$
(\bar{D} \backslash D) \cap \bigcup_{x \in X} U_{x}=\emptyset
$$

where the closure of $D$ is taken in $K$. So in particular if $\mathfrak{p}>|X|$, such a subset $Y$ of $X$ cannot be separable. This establishes the following.

Theorem 23.2 ([102]). Assuming PID, every regular hereditarily separable space of cardinality $<\mathfrak{p}$ is Lindelöf

So, in particular, assuming PID and $\mathfrak{p}>\omega_{1}$, we conclude that every regular hereditarily separable space is Lindelöf, i.e. we have a solution to the S-space problem. However, examining more closely the above argument one realizes that this argument is really about the ambient spaces $K$ that have some compactness as well as convergence properties leading us eventually to the following interesting result.

Theorem 23.3 ([9], 41]). Assuming PFA, every compact countably tight space is sequential.

Using a forcing and absoluteness argument this has the following fact 53 as an immediate consequence.

Theorem 23.4. Assuming PFA, every compact countably tight space has a $G_{\delta^{-}}$ point and is therefore sequentially compact.

\footnotetext{
${ }^{52} \mathrm{It}$ is here that we were originally using the dual form of PID discussed above and saying that for an $\aleph_{1}$-generated ideal $\mathcal{I}$ on some uncountable set $S$ either there is an uncountable subset of $S$ orthogonal to $\mathcal{I}$ or an uncountable subset $X$ of $S$ such that $[X]^{\aleph_{0}} \subseteq I$.

${ }^{53}$ This fact has been first observed by Alan Dow.
} 
Looking at the analogues of these results in the context of descriptive set theory one arrives at the following result whose proof however requires some Ramsey theory (see [129; Ch. 7).

Theorem 23.5. Every countable analytic spac 54 with a countably tight compactification is bisequentia 55

Typical examples of countable analytic spaces are countable collections of Borel functions on some Polish, or more generally, completely metrizable space. The following well known result connects this with the theory of compact countably tight spaces.

Theorem 23.6 ([86]). Every compact set of Baire-class-1 functions defined on some Polish space is countably tight and sequentially compact.

Combining Theorems 23.5 and 23.6 we get the following well-known result.

Theorem 23.7 ([14]). Every compact set of Baire-class-1 functions defined on some Polish space is Fréche 56 .

Example 23.1 and Theorem 23.2 suggest the following natural question.

Question 23.8. Are any of the following statements equivalent under PID?

(1) Every regular hereditarily separable space is Lindelöf.

(2) Every regular first countable hereditarily separable space is Lindelöf.

(3) Every compact hereditarily separable space is hereditarily Liindelöf.

(4) $\mathfrak{b}=\omega_{2}$.

(5) $\mathfrak{p}=\omega_{2}$.

We finish this section by mentioning that the dual problem, the L-space problem, has a negative solution.

Theorem 23.9 ([7]). There is a regular hereditarily Lindelöf nonseparable space.

\section{PiD and Classification of Transitive Relations on $\omega_{1}$}

\subsection{Tukey Reductions and Cofinal Types.}

Definition 24.1. For two posets $D$ and $E$, set

$$
D \leq_{T} E \text { iff }(\exists f: D \rightarrow E)\left[X \text { is unbounded in } D \Rightarrow f^{\prime \prime} X \text { is unbounded in } E\right. \text {.] }
$$

The map $f: D \rightarrow E$ witnessing $D \leq_{T} E$ is called a Tukey-map or Tukey-reduction. Put

$$
D \equiv_{T} E \text { iff } D \leq_{T} E \text { and } E \leq_{T} D .
$$

Equivalence classes of $\equiv_{T}$ are called Tukey types. We shall let $D<_{T} E$ denote the fact that $D \leq_{T} E$ but not $E \leq_{T} D$.

Proposition 24.2 ([131]). The following are equivalent for two posets $D$ and $E$ :

(1) $D \leq_{T} E$,

\footnotetext{
${ }^{54} \mathrm{~A}$ topological space $X$ is analytic if its topology is analytic as a subset of $2^{X}$.

${ }^{55}$ Recall that a space $X$ is bisequential if every ultrafilter $\mathcal{U}$ on $X$ converging to a point $x$ has a subsequence $\left\{A_{n}: n<\omega\right\} \subseteq \mathcal{U}$ converging to $x$.

${ }^{56}$ Recall that a space $X$ is Fréchet if every subset of $X$ which accumulates to a point of $X$ contains a sequence which converges to the point.
} 
(2) There is a convergent map $g: E \rightarrow D$, i.e., a map with the property that for every $d \in D$ there is $e \in E$ such that $g\left(e^{\prime}\right) \geq d$ for all $e^{\prime} \geq e$,

(3) There is a cofinal map $h: E \rightarrow D$, i.e., a map which maps cofinal subsets of $E$ to cofinal subsets of $D$

Remark 24.3. It is for this reason that sometimes Tukey-types are also called cofinal types. Another reason is the following interesting result.

Theorem 24.4 ([131]). The following conditions are equivalent for every pair $D$ and $E$ of directed sets:

(1) $D \equiv_{T} E$,

(2) $D$ and $E$ can be isomorphically embedded as cofinal subsets of a third directed poset $X$.

\subsection{The Five Tukey Types.}

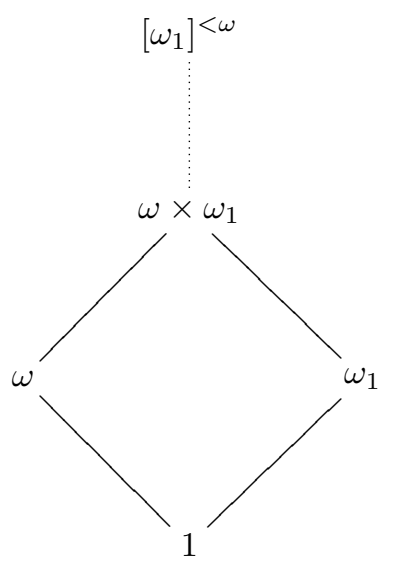

Question 24.5 ([131]). Is there any other Tukey type below $\left[\omega_{1}\right]^{<\omega}$ ?

The first answer to this question given in 52] while incomplete did (at least implicitly) point towards the Tukey-classification theory of definable directed sets, an active area of current research in set theory (see [42], 95]).

Theorem 24.6 ([52]). If $\mathrm{CH}$ holds there exist at least seven Tukey types of directed posets of cardinality at most $\aleph_{1}$. For example, the lattices $\mathbb{N}^{\mathbb{N}}$ and $\ell_{1}$ are pairwise Tukey-inequivalent and also not Tukey-equivalent to any of the five basic directed sets $1, \omega, \omega_{1}, \omega \times \omega_{1}$, and $\left[\omega_{1}\right]^{<\omega}$.

A more general construction can be obtained as follows. To a given topological space $X$ we associate a directed set as follows

$$
\mathcal{K}(X)=\{K \subseteq X: K \text { compact }\} .
$$

It turns out that $\mathcal{K}(X)$ for $X$ a subspace of $\omega_{1}$ (equipped with its natural order topology) realizes many different Tukey types of directed sets of cardinality continuum. In particular, we have the following fact showing that it is not reasonable to expect that we can identify all cofinal types of directed sets of cardinality continuum. 
Theorem 24.7 ([106]). For uncountable $A, B \subseteq \omega_{1}$, if there is a Tukey reduction from $\mathcal{K}(A)$ into $\mathcal{K}(B)$ then $B \backslash A$ must be non-stationary. So if for two uncountable subsets $A$ and $B$ of $\omega_{1}$ have stationary symmetric difference, the corresponding directed sets $\mathcal{K}(A)$ and $\mathcal{K}(B)$ are not Tukey-equivalent.

Example 24.8 ([107]). If $\mathfrak{b}=\omega_{1}$, there is a sublattice $D_{\mathfrak{b}}$ of $\mathbb{N}^{\mathbb{N}}$ such that

$$
\omega \times \omega_{1}<_{T} D_{\mathfrak{b}}<_{T}\left[\omega_{1}\right]^{<\omega} .
$$

For example, let $D_{\mathfrak{b}}$ be the sublattice of $\mathbb{N}^{\mathbb{N}}$ generated by an $<^{*}$-increasing $<^{*}$ unbounded sequence

$$
\left\{f_{\xi}: \xi<\mathfrak{b}\right\} \subseteq \mathbb{N}^{\uparrow \mathbb{N}} .
$$

Definition 24.9. A subset of $D$ is strongly unbounded if it is infinite and if all of its infinite subsets are unbounded.

Lemma 24.10. In any poset of cofinality $<\mathfrak{b}$ the class of countable strongly unbounded sets forms a P-ideal.

Proof. Consider a sequence $A_{n}(n<\omega)$ of countable strongly unbounded subsets of $D$. Then for each $d \in D$ there is $f_{d} \in \mathbb{N}^{\mathbb{N}}$ such that

$$
\left(\bigcup_{n<\omega} A_{n}\left(f_{d}(n)\right)\right) \cap\left\{x \in D: x \leq_{D} d\right\}=\emptyset,
$$

where $A_{n}(k)$ is our notation for the set of first $k$ elements of $A_{n}$ in some fixed enumeration of this set in type $\omega$. Choose $g \in \mathbb{N}^{\mathbb{N}}$ such that $\left\{d \in D: f_{d}<^{*} g\right\}$ is cofinal in $D$. Then $B=\bigcup_{n<\omega} A_{n}(g(n))$ is a strongly unbounded subset of $D$ such that $A_{n} \subseteq^{*} B$ for all $n$.

Definition 24.11. A subset of $D$ is pseudo-bounded if it contains no strongly unbounded subsets, or equivalently if every infinite subset of $D$ contains an infinite bounded subset.

Theorem 24.12 ([106]). Assuming PID, for every directed (or undirected) poset $D$ of cofinality $<\mathfrak{b}$, either

(1) $\left[\omega_{1}\left[<\omega \leq_{T} D\right.\right.$, or

(2) $D$ can be decomposed into countably many pseudo-bounded subsets.

This suggests considering the class $\mathcal{D}_{0}$ of all directed sets $D$ with the property that pseudo-bounded countable subsets of $D$ are in fact bounded.

Theorem 24.13 ([106]). The following statements are equivalent assuming PID:

(1) $1, \omega, \omega_{1}, \omega \times \omega_{1}$, and $\left[\omega_{1}\right]<\omega$ are all Tukey types of directed sets belonging to the class $\mathcal{D}_{0}$ and having cofinality $\leq \omega_{1}$;

(2) $\mathfrak{b}=\omega_{2}$.

Question 24.14. Assuming PID, is $\mathfrak{b}=\omega_{2}$ equivalent to the statement that

$$
1, \omega, \omega_{1}, \omega \times \omega_{1} \text {, and }\left[\omega_{1}\right]^{<\omega}
$$

represent all the Tukey-types of directed sets of size $\aleph_{1}$ ?

Lemma 24.15. In a directed set $D$ of cofinality $<\mathfrak{p}$, every countable pseudobounded subset of $D$ is in fact bounded. 
Proof. Consider a countable unbounded subset $A$ of $D$ and fix a cofinal subset $D^{\prime}$ of $D$ of cardinality $<\mathfrak{p}$. Then $A \backslash\left\{x \in D: x \leq_{D} d\right\},\left(d \in D^{\prime}\right)$ is a downwards directed family of infinite subsets of $A$ of size $<\mathfrak{p}$ so we can find infinite $B \subseteq A$ which is almost included in every member of the family. It follows that $B$ is strongly unbounded in $D$ and so in particular $A$ is not pseudo-bounded.

Theorem 24.16 ([106]). Assuming PID and the equality $\mathfrak{p}=\omega_{2}$,

$$
1, \omega, \omega_{1}, \omega \times \omega_{1} \text {, and }\left[\omega_{1}\right]^{<\omega}
$$

represent all Tukey-types of directed sets of cardinality at most $\aleph_{1}$.

Question 24.17. Assuming PID, is $\mathfrak{p}=\omega_{2}$ equivalent to the statement that

$$
1, \omega, \omega_{1}, \omega \times \omega_{1} \text {, and }\left[\omega_{1}\right]^{<\omega}
$$

represent all the Tukey-types of directed sets of size $\aleph_{1}$ ?

A similar result holds for all posets of cardinality at most $\aleph_{1}$ though the list of possible Tukey types is countably infinite. To succinctly state this result, let

$$
D_{0}=1, D_{1}=\omega, D_{2}=\omega_{1}, D_{3}=\omega \times \omega_{1} \text { and } D_{4}=\left[\omega_{1}\right]^{<\omega_{1}}
$$

be the ordered list of basic five directed posets of size at most $\aleph_{1}$. We also need to recall that $\mathfrak{m}$ is the minimal cardinal $\kappa$ for which one can find a ccc poset $\mathcal{P}$ and $\kappa$ dense open subsets of $\mathcal{P}$ such that no filter of $\mathcal{P}$ meets all the dense sets, or equivalently $\mathfrak{m}$ is the minimal cardinality of a ccc non- $\sigma$-centered poset. Then $\mathfrak{m} \leq \mathfrak{p} \leq \mathfrak{b}$, so in the context of PID the cardinal $\mathfrak{m}$ has only two possible values, $\mathfrak{m}=\omega_{1}$ or $\mathfrak{m}=\omega_{2}$. So in the context of PID, the statement $\mathfrak{m}=\omega_{2}$ is equivalent to $\mathrm{MA}_{\aleph_{1}}$. We direct the reader to [109] and [112] for more information about $\mathfrak{m}$.

Theorem 24.18 ([117]). The following statements are equivalent assuming PID:

(1) $\mathfrak{m}=\omega_{2}$.

(2) Every poset of size $\aleph_{1}$ is Tukey-equivalent to one from the list:

(a) $\bigoplus_{i<5} n_{i} D_{i}\left(i<5, n_{i}<\omega\right)$,

(b) $\aleph_{0} \cdot 1 \oplus \bigoplus_{i=2}^{4} n_{i} D_{i}\left(2 \leq i<5, n_{i}<\omega\right)$,

(c) $\aleph_{0} \cdot \omega_{1} \oplus n_{4}\left[\omega_{1}\right]^{<\omega}\left(n_{4}<\omega\right)$,

(d) $\aleph_{0} \cdot\left[\omega_{1}\right]^{<\omega}$,

(e) $\aleph_{1} \cdot 1$.

This leads us to the following question.

Question 24.19. Are any of the three statements $\mathfrak{b}=\omega_{2}, \mathfrak{p}=\omega_{2}$ and $\mathfrak{m}=\omega_{2}$ equivalent under PID?

\section{PiD and Weakly Distributive Boolean Algebras}

Recall the following well known condition on a complete Boolean algebra.

Definition 25.1. A Boolean algebra $\mathbb{B}$ is weakly distributive if for every sequence

$$
\begin{gathered}
\left\{a_{n k}: n, k<\omega\right\} \subseteq \mathbb{B}, \\
\bigwedge_{n} \bigvee_{k} a_{n k}=\bigvee_{F} \bigwedge_{n} a_{n F(n)},
\end{gathered}
$$

where $F \in$ Fin $^{\omega}$, and where for a finite set $X \subseteq \omega$,

$$
a_{n X}=\bigvee_{k \in X} a_{n k}
$$


Particularly interesting are complete weakly distributive Boolean algebras satisfying the countable chain condition. The Souslin algebra, the algebra of regular-open subsets of the ccc nowhere separable linearly ordered continuum, is such an algebra, a fact first pointed out in [71. The existence of these algebras is easily seen to be in fact equivalent to the negation of Souslin hypothesis and so the following fact rules out their existence inder PID.

Theorem 25.2 ([2]). The P-ideal dichotomy implies the Souslin hypothesis.

Proof. Given a Souslin tree $T$, let

$$
\mathcal{I}_{T}=\left\{a \in[T]^{\leq \aleph_{0}}: a \text { is strongly unbounded in } T\right\} .
$$

Claim 1. $\mathcal{I}_{T}$ is a P-ideal (i.e. we don't need to assume $\mathfrak{b}>\omega_{1}$ ).

Proof. Let $\left\{a_{n}: n<\omega\right\} \subseteq \mathcal{I}_{T}$ be given. Fix $\delta<\omega_{1}$ such that $\bigcup_{n<\omega} a_{n} \subseteq T\lceil\delta$ and let $\left\{t_{k}: k<\omega\right\}=T_{\delta}$. Then for each $k, n<\omega$, the set $a_{n}(k)=\left\{s \in a_{n}: s<_{T} t_{k}\right\}$ is finite. Let $a=\bigcup_{n<\omega}\left(a_{n} \backslash \bigcup_{k \leq n} a_{n}(k)\right)$. Then $a \in \mathcal{I}_{T}$ and $a \supseteq^{*} a_{n}$ for all $n<\omega$.

Applying PID to $\mathcal{I}_{T}$, we have that either:

(1) $T$ contains an uncountable strongly unbounded set $X$. Impossible, because $X$ would in particular contain an uncountable antichain.

(2) $T$ can be covered with countably many pseudo-bounded subsets. Impossible, because a pseudo-bounded subset of $T$ can be covered by finitely many chains of $T$. So in particular, $T$ would have an uncountable chain.

Theorem 25.3 ([50]). PID implies that the Souslin hypothesis gets preserved under forcing by a measure algebra.

Proof. Fix a measure algebra $\mathcal{R}$ and an $\mathcal{R}$-name $\dot{\leq}_{T}$ such that

$$
\llbracket\left(\check{\omega}_{1}, \dot{\leq}_{T}\right) \text { is a Souslin tree } \rrbracket=\mathbb{1}
$$

Let

$$
\mathcal{J}=\left\{a \in\left[\omega_{1}\right]^{\leq \aleph_{0}}: \llbracket \check{a} \in \dot{\mathcal{I}}_{T} \rrbracket=\mathbb{1}\right\} .
$$

Claim 1. $\mathcal{J}$ is a P-ideal.

Proof. Consider $\left\{a_{n}: n<\omega\right\} \subseteq \mathcal{J}$ and fix $\delta<\omega_{1}$ such that $\llbracket \bigcup_{n<\omega} \check{a}_{n} \subseteq \check{\delta} \rrbracket=\mathbb{1}$. We assume that $\llbracket[\delta, \delta+\omega)$ is the $\delta^{t h}$ level $\rrbracket=\mathbb{1}$. Let $\dot{a}_{n k}$ be the $\mathcal{R}$-name for the set $\left\{s \in a_{n}: s<_{\dot{T}} \delta+k\right\}$. For $n, m<\omega$, find a finite set $F_{n m} \subseteq a_{n}$ with the property that $\mu\left(\llbracket \bigcup_{k \leq n} \dot{a}_{n k} \subseteq \check{F}_{n m} \rrbracket\right)>1-2^{-m}$. Finally, let

$$
a=\bigcup_{m<\omega}\left(a_{m} \backslash \bigcup_{n \leq m} F_{n m}\right) .
$$

For $m<\omega$, set $a^{m}=a \cap \bigcup_{n \geq m} a_{n}$. Then for all $m \geq k$,

$$
\mu\left(\llbracket \operatorname{pred}(\delta+k) \cap a^{m} \neq \emptyset \rrbracket\right)<2^{-m+1}
$$

so we must have that $\llbracket a$ is strongly unbounded $\rrbracket=\mathbb{1}$.

Applying PID, we have the following alternatives:

(1) There is uncountable $X \subseteq \omega_{1}$ such that $\llbracket[X]^{\aleph_{0}} \subseteq \mathcal{I}_{T} \rrbracket=\mathbb{1}$. In this case,

$$
\llbracket\left(\omega_{1}, \leq_{\dot{T}}\right) \text { has an uncountable antichain } \rrbracket=\mathbb{1} .
$$


(2) There is uncountable $Y \subseteq \omega_{1}$ such that $Y \perp \mathcal{J}$. In this case one shows that some condition of $\mathcal{R}$ forces that $\left(\omega_{1}, \leq_{\dot{T}}\right)$ has an uncountable branch.

Definition 25.4. A subset $X$ of some poset $\mathbb{P}$ is essentially strongly unbounded if

$$
\{p \in \mathbb{P}:\{x \in X: p \leq x\} \in \operatorname{Fin}\}
$$

is dense-open in $\mathbb{P}$.

Note 25.5. If $\mathbb{P}$ is a (normal) tree of height, say $\omega_{1}$, then essentially strongly unbounded sets are strongly unbounded.

Proposition 25.6 ([82, [7]). If $\mathbb{P}$ is an $\omega^{\omega}$-bounding ccc poset then the ideal $\mathcal{I}_{\mathbb{P}}$ of all countable essentially strongly unbounded subsets of $\mathbb{P}$ is a P-ideal.

Remark 25.7. If $\mathbb{P}=\mathbb{B} \backslash\{\mathbb{O}\}$ for some Boolean algebra $\mathbb{B}$, the $\omega^{\omega}$-boundedness of $\mathbb{P}$ is the same as the weak distributivity of the Boolean algebra $\mathbb{B}$.

Definition 25.8. A function $\nu: \mathbb{B} \rightarrow[0, \infty)$ is a strictly positive continuous submeasure on $\mathbb{B}$ if

(1) $\nu(a)=0$ iff $a=\mathbb{0}$,

(2) $a \leq b$ implies $\nu(a) \leq \nu(b)$,

(3) $\nu(a \vee b) \leq \nu(a)+\nu(b)$

(4) $a_{n} \downarrow \mathbb{O}$ implies $\nu\left(a_{n}\right) \rightarrow 0$,

If (3) is replaced by a stronger condition

$(3)^{*} \nu(a \vee b)=\nu(a)+\nu(b)$ whenever $a \wedge b=\mathbb{0}$,

then $\nu$ is a $\sigma$-additive measure (strictly positive as well).

The following problem is a special case of the problem asking for conditions that would guarantee the existence of strictly positive continuous submeasures on complete Boolean algebras (see, [8]).

Problem 25.9 ( $[72]^{57}$ ). Does every complete weakly distributive ccc algebra support a strictly positive $\sigma$-additive measure?

This problems splits naturally into the following two parts second of which became known in the literature under the name of 'the control measure problem'.

Problem 25.10 ([71]).

(1) Does every weakly distributive B satisfying the ccc support a strictly positive continuous submeasure?

(2) Given that B supports a strictly positive continuous submeasure, does it also support a strictly positive $\sigma$-additive measure?

Theorem 25.11 (7). The PID implies that every complete weakly distributive algebra $\mathbb{B}$ satisfying the ccc supports a strictly positive continuous submeasure.

Proof Sketch. Apply PID to the P-ideal $\mathcal{I}_{\mathbb{B}}$ of all countable essentially strongly unbounded subsets of $\mathbb{B} \backslash\{0\}$, which can also be viewed as the collection of

$$
\left\{x_{n}: n<\omega\right\} \subseteq \mathbb{B}^{+}
$$

such that

$$
\bigwedge_{m} \bigvee_{n \geq m} x_{n}=\mathbb{0}
$$

${ }^{57}$ Problem 163, put down originally by von Neumann on July 4, 1937. 
i.e., sequences converging to $\mathbb{O}$. Note that since $\mathbb{B}$ is ccc there is no uncountable $\mathcal{X} \subseteq \mathbb{B} \backslash\{\mathbb{O}\}$ such that $[\mathcal{X}]^{\aleph_{0}} \subseteq \mathcal{I}_{\mathbb{B}}$ and note that the second alternative of PID means that $\mathbb{O}$ is a $G_{\delta}$-point of $\mathbb{B}$ in the sequential order topology of $\mathbb{B}$. Then use the result of [6] to show that the sequential order topology of $\mathbb{B}$ is in fact metrizable.

Remark 25.12. More details about the Von Neumann and Maharam problems can be found in articles 8 and 134 . We shall now work towards removing the use of PID from the analysis of this problem.

Definition 25.13 (51]). A Boolean algebra satisfies the $\sigma$-finite chain condition if it can be decomposed as

$$
\mathbb{B}=\bigcup_{n<\omega} \mathbb{B}_{n}
$$

such that no $\mathbb{B}_{n}$ contains an infinite subset of pairwise disjoint elements.

Theorem 25.14 ([125]). The following are equivalent for every complete Boolean algebra $\mathbb{B}$ :

(1) $\mathbb{B}$ carries a strictly positive continuous submeasure;

(2) (a) $\mathbb{B}$ is weakly distributive, and

(b) B satisfies the $\sigma$-finite chain condition.

Remark 25.15. As the proof of [125] shows, the $\sigma$-finite chain condition in Theorem 25.14 can be replaced by any kind of countable chain condition which is preserved by the proper poset (which could also be assumed not to add reals) forcing an instance of the P-ideal dichotomy. For example, the countable chain condition of any complete Boolean algebra generated by an appropriately definable poset on a Polish space satisfies this requirement, so it will have a strictly positive continuous measure whenever it is weakly distributive (see [32]).

We finish this setion with a result which shows that the second part of Problem 25.10 has a negative answer.

Theorem 25.16 ([100]). There is a complete Boolean algebra $\mathbb{B}$ carrying a strictly positive continuous submeasure which supports no strictly positive $\sigma$-additive measure.

\section{PID and Biorthogonal Systems in Banach spaces}

Recall that a biorthogonal system in some Banach space $X$ is any sequence $\left\{\left(x_{i}, f_{i}\right): i \in I\right\} \subseteq X \times X^{*}$ such that $f_{i}\left(x_{j}\right)=\delta_{i j}$, where $\delta_{i j}$ is Kronecker's delta. This concept has many variations and is related to many problems of this area of functional analysis (see [46]). For example, the problem whether this concept characterizes the separability of Banach spaces, i.e. whether every nonseparable Banach space has an uncountable biorthogonal system, has been around for quite some time (see [19]). The following example points out towards the set-theoretical nature of this question and hints that PID might be relevant.

Example 26.1 ([108). If $\mathfrak{b}=\omega_{1}$ there is a compact nonmetrizable scattered space $K$ such that its function space $X=C(K)$ is hereditarily Lindelöf relative to its weak topology and so in particular $X$ has no uncountable biorthogonal system.

It turns out that the right way to look at this problem is to work towards showing that under certain assumptions the space $X$ has a quotient with a Schauder basis of length $\omega_{1}$. 
Theorem 26.2 ([126]). Assuming PID, the following conditions are equivalent for every Banach space $X$ of density $<\mathfrak{p}$ :

(1) $X$ can be written as the union of a strictly increasing $\omega_{1}$-sequence of closed subspaces.

(2) There is a bounded linear operator $T: X \rightarrow c_{0}\left(\omega_{1}\right)$ with nonseparable range.

The condition (1) means that there is a sequence $f_{\xi}\left(\xi<\omega_{1}\right)$ of norm- 1 functionals such that

$$
(\forall \varepsilon>0)(\forall x \in X)\left\{\xi<\omega_{1}:\left|f_{\xi}(x)\right| \geq \varepsilon\right\} \text { is countable. }
$$

We get the condition (2) by finding an uncountable set $\Gamma \subseteq \omega_{1}$ such that

$$
(\forall \varepsilon>0)(\forall x \in X)\left\{\xi \in \Gamma:\left|f_{\xi}(x)\right| \geq \varepsilon\right\} \text { is finite. }
$$

To this end, let

$$
\mathcal{I}=\left\{A \in\left[\omega_{1}\right]^{\leq \aleph_{0}}:(\forall \varepsilon>0)(\forall x \in X)\left\{\xi \in A:\left|f_{\xi}(x)\right| \geq \varepsilon\right\} \text { is finite }\right\} .
$$

By our assumption on density of $X$, the ideal $\mathcal{I}$ is a P-ideal. Clearly, the first alternative of PID is giving us the desired conclusion so we examine the second. Suppose $\Gamma$ is an uncountable subset of $\omega_{1}$ orthogonal to $\mathcal{I}$. Then again using our assumption that $\mathfrak{p}$ is bigger that the density of $X$, we conclude that, relative to the weak*-topology of $X^{*}$, the set $H=\left\{f_{\xi}: \xi \in \Gamma\right\}$ accumulates to $0^{*}$ though no countable subset of $H$ does. Let

$$
K=\bigcup_{\alpha<\omega_{1}} \overline{\left\{f_{\xi}: \xi \in \Gamma \cap \alpha\right\}},
$$

where the closure is taken relative to the weak*-topology. Let

$$
R=\{f-g: f, g \in K, f \neq g\} .
$$

We repeat the procedure by defining now the ideal

$$
\mathcal{J}=\left\{A \in[R]^{\leq \aleph_{0}}:(\forall \varepsilon>0)(\forall x \in X)\{f \in A:|f(x)| \geq \varepsilon\} \text { is finite }\right\} .
$$

Again this is a P-ideal and the first alternative of PID is giving the desired conclusion, so we finish the proof with the following claim.

Claim 1. The set $R$ cannot be covered by countably many subsets orthogonal to $\mathcal{J}$.

The relevance of condition (1) of Theorem 26.2 is explained by the following fact.

Theorem 26.3 ([126]). Assuming PID, the following conditions are equivalent for every Banach space $X$ of density $<\mathfrak{m}$ :

(1) There is a bounded linear operator $T: X \rightarrow c_{0}\left(\omega_{1}\right)$ with nonseparable range.

(2) There is a quotient map $Q: X \rightarrow Y$ onto a Banach space $Y$ with a monotone Schauder basis of length $\omega_{1}$.

Corollary 26.4 ([126]). Assuming PID and $\mathfrak{m}>\omega_{1}$, every nonseparable Banach space has an uncountable biorthogonal system.

The operators $T: X \rightarrow c_{0}\left(\omega_{1}\right)$ with nonseparable range are usually given as

$$
T(x)=\left(f_{\xi}(x)\right)_{\xi<\omega_{1}},
$$

where $f_{\xi}\left(\xi<\omega_{1}\right)$ is a sequence of uniformly bounded functionals. The proof of Theorem 26.3 gives us the quotient $Y$ with a Schauder basis $\left(e_{\alpha}\right)_{\alpha<\omega_{1}}$ such that the corresonding sequence $\left(e_{\alpha}^{*}\right)_{\alpha<\omega_{1}}$ of biorthogonal functionals is really a subsequence of the given sequence $f_{\xi}\left(\xi<\omega_{1}\right)$ which defines the operator $T$. It is for this reason that Theorem 26.3 (and its proof) has particularly strong influence on Banach 
spaces of density $\aleph_{1}$. For example, in this case the second alternative of Theorem 26.3 will give us not only uncountable but also fundamental biorthogonal system $\left\{\left(x_{\alpha}, g_{\alpha}\right): \alpha<\omega_{1}\right\}$, i.e a biorthogonal system such that $X=\overline{\operatorname{span}}\left\{x_{\alpha}: \alpha<\omega_{1}\right\}$. In fact, going through the proof of a standard result in this area (Theorem 4.15 of [46]), we see that the sequence $\left(g_{\alpha}\right)_{\alpha<\omega_{1}}$ can be chosen to be a subsequence of $\left(f_{\xi}\right)_{\xi<\omega_{1}}$ as well. This gives us the following corollary to be used later.

Corollary 26.5. Assume PID and $\mathfrak{m}>\omega_{1}$. Let $X$ be a Banach space of density $\aleph_{1}$ and that for some bounded sequence $\left\{f_{\xi}: \xi<\omega_{1}\right\} \subseteq X^{*}$ the operator $T(x)=\left(f_{\xi}(x)\right)_{\xi<\omega_{1}}$ maps $X$ into a nonseparable subset of $c_{0}\left(\omega_{1}\right)$. Then $X$ has a fundamental biorthogonal system $\left\{\left(x_{\alpha}, g_{\alpha}\right): \alpha<\omega_{1}\right\}$ with $\left(g_{\alpha}\right)_{\alpha<\omega_{1}}$ a subsequence of $\left(f_{\xi}\right)_{\xi<\omega_{1}}$.

We finish this section with two applications of Theorem 26.3. Recall that a point $x$ of closed convex subset $C$ of $X$ is its support point if there is $f \in X^{*}$ such that

$$
f(x)=\inf _{y \in C}<\sup _{y \in C} f(y) .
$$

In [85, Rolewicz investigated the existence of closed convex sets supported by all of its points. He noticed that no separable Banach space can contain such a convex subset and after noting that many nonseparable Banach space do contain such sets asked if this is true for all Banach spaces. It is known that some generic nonseparable Banach spaces fail to have convex subsets supported by all of their points (see [12, 69], 66]).

Theorem 26.6 ([126]). Assuming PID and $\mathfrak{m}>\omega_{1}$, every nonseparable Banach space contains a closed convex subset supported by all of its points.

Proof. Let $\left\{\left(x_{i}, f_{i}\right): i \in I\right\}$ be an uncountable biorthogonal system in some Banach space $X$. Let $C=\overline{\operatorname{conv}}\left\{x_{i}: i \in I\right\}$, the closed convex hull of the points from the biorthogonal system. Then it is easily checked that every point of $C$ is its support point.

Example 26.1 and Corollary 26.4 suggest the following natural question.

Question 26.7. Are the following statement equivalent under PID?

(1) Every nonseparable Banach space has an uncountable biorthogonal system.

(2) $\mathfrak{b}=\omega_{2}$.

The second application is to a purely geometric problem first investigated by Mazur 73 who proved that if a Banach space $X$ has a Fréchet differentiable norm then every closed convex subset of $X$ is the intersection of closed balls of $X$ (or, in short, the norm has the Mazur intersection property). The Mazur intersection property is indeed closely related to the differentiability in the context of Banach spaces and for this reason is studied only in the context of spaces in which every convex continuous function is Fréchet differentiable on a dense set of points, or equivalently in the class of Banach spaces $X$ with the property that every separable subspace of $X$ has separable dual. This is a well studied class of Banach spaces known in the literature as the class of strong differentiability spaces or Asplund spaces (see, for example, 28] and [20]). The close connection between the Mazur intersection property and biorthogonal systems is revealed by the following two results. 
Theorem 26.8. [56] Suppose that a Banach space $X$ has a biorthogonal system $\left\{\left(x_{i}, f_{i}\right): i \in I\right\} \subseteq X \times X^{*}$ such that $X^{*}=\overline{\operatorname{span}}\left\{f_{i}: i \in I\right\}$. Then $X$ admits an equivalent norm with the Mazur intersection property.

Theorem 26.9. [56] Suppose that a nonseparable Banach space $X$ admits an equivalent norm with the Mazur intersection property. Then for every $\varepsilon>0$ there is an uncountable $\varepsilon$-biorthogonal system $\left\{\left(x_{\xi}, f_{\xi}\right): \xi<\omega_{1}\right\} \subseteq X \times X^{*}$, i.e. a system such that $f_{\xi}\left(x_{\xi}\right)=1$ and $\left|f_{\xi}\left(x_{\eta}\right)\right| \leq \varepsilon$ for $\xi \neq \eta$.

So, in particular, the space $X=C(K)$ of Example 26.1 does not admit renorming with the Mazur intersection property and it is, therefore, natural to investigate the relevance of PID to the Mazur problem. This is done using the following result which shows that the class of Asplund spaces of density $\aleph_{1}$ satisfies the hypothesis of Corollary 26.5

Lemma 26.10 (5]). Suppose $X$ is an Asplund space of density $\aleph_{1}$ and with an uncountable biorthogonal system. Then there is a sequence $\left\{y_{\xi}: \xi<\omega_{1}\right\} \subseteq S_{X}$ such that the operator $f \mapsto\left(f\left(y_{\xi}\right)\right)_{\xi<\omega_{1}}$ maps $X^{*}$ into a nonseparable subset of $c_{0}\left(\omega_{1}\right)$.

Combining Corollaries 26.4 and 26.5, Theorem 26.8 and Lemma 26.10, we get the following interesting solution to the Mazur problem.

Theorem 26.11 ([5]). Assuming PID, every Asplund space $X$ of density $<\mathfrak{m}$ admits an equivalent norm such that every closed convex subset of $X$ is the intersection of closed balls relative to the new norm.

\section{REFERENCES}

[1] Uri Abraham, Matatyahu Rubin and Saharon Shelah, On the consistency of some partition theorems for continuous colorings, and the structure of $\aleph_{1}$-dense real order types. Ann. Pure Appl. Logic 29 (1985), no. 2, 123-206.

[2] Uri Abraham and Stevo Todorcevic, Partition properties of $\omega_{1}$ compatible with CH, Fundamenta Mathematicae 152 (1997), no. 2, pp. 165-181.

[3] Spiros A. Argyros, Pandelis Dodos and Vassilis Kanellopoulos, Unconditional families in Banach spaces. Math. Ann. 341 (2008), no. 1, 15-38.

[4] Antonio Avilés and Stevo Todorcevic, Multiple gaps, arXiv:1001.4888

[5] Miroslav Bačák, Petr Hájek, Mazur intersection property for Asplund spaces. J. Funct. Anal. 255 (2008), no. 8, 2090-2094.

[6] Bohuslav Balcar, Wieslaw Glwczynski, Thomas Jech, The sequential topology on complete Boolean algebras. Fund. Math. 155 (1998), no. 1, 59-78.

[7] Bohuslav Balcar, Thomas J. Jech, and T. Pazák, Complete CCC Boolean algebras, the order sequential topology, and a problem of von Neumann, The Bulletin of the London Mathematical Society 37 (2005), no. 6, pp. 885-898.

[8] Bohuslav Balcar and Thomas Jech, Weak distributivity, a problem of von Neumann and the mystery of measurability. Bull. Symbolic Logic 12 (2006), no. 2, 241-266.

[9] Zoltán T. Balogh, On compact Hausdorff spaces of countable tightness. Proc. Amer. Math. Soc. 105 (1989), no. 3, 755-764.

[10] James E. Baumgartner and Alan D. Taylor, Saturation properties of ideals in generic extensions. I. Trans. Amer. Math. Soc. 270 (1982), no. 2, 557-574.

[11] Mohamed Bekkali, Topics in set theory. Lebesgue measurability, large cardinals, forcing axioms, rho-functions. Notes on lectures by Stevo Todorcevic. Lecture Notes in Mathematics, 1476. Springer-Verlag, Berlin, 1991. viii+120 pp.

[12] Murray Bell, John Ginsburg, Stevo Todorcevic, Countable spread of $\exp Y$ and $\lambda Y$. Topology Appl. 14 (1982), no. 1, 1-12.

[13] Andreas Blass, A partition theorem for perfect sets. Proc. Amer. Math. Soc. 82 (1981), no. 2, 271-277. 
[14] Jean Bourgain, David H. Fremlin and Michael Talagrand, Pointwise compact sets of Bairemeasurable functions. Amer. J. Math. 100 (1978), no. 4, 845-886.

[15] Lawrence G. Brown, Ronald George Douglas, and Peter A. Fillmore, Extensions of $C^{*}$ algebras and K-homology, Annals of Mathematics, Second Series 105 (March, 1977), no. 2, pp. 265-324.

[16] Tim Carlson and Richard Laver, Sacks reals and Martin's axiom, Fundamenta Mathematicae, 133 (1989), 161-168.

[17] Jens Peter Reus Christensen, Topology and Borel structure. North-Holland Publishing Co., Amsterdam-London 1974. iii+133 pp.

[18] Jens Peter Reus Christensen, Some results with relation to the control measure problem. Vector space measures and applications (Proc. Conf., Univ. Dublin, Dublin, 1977), II, pp. 27-34, Lecture Notes in Phys., 77, Springer, Berlin-New York, 1978.

[19] William J.Davis, William B. Johnson, On the existence of fundamental and total bounded biorthogonal systems in Banach spaces. Studia Math. 45 (1973), 173-179.

[20] Robert Deville, Gilles Godefroy, Václav Zizler, Smoothness and renormings in Banach spaces. John Wiley \& Sons, Inc., New York, 1993. xii+376 pp. ISBN: 0-582-07250-6

[21] Robert Palmer Dilworth, A decomposition theorem for partially ordered sets, Annals of Mathematics, Second Series 51 (Jan., 1950), no. 1, pp. 161-166.

[22] Philipp Doebler, Rado's Conjecture implies that all stationary set preserving forcings are semiproper. preprint 2010.

[23] Philipp Doebler and Ralf Schindler, $\Pi_{2}$ consequences of $\mathrm{BMM}$ plus $\mathrm{NS}_{\omega_{1}}$ is precipitous and the semiproperness of statioary set preserving forcings, Math. Res. Lett., 16 (2009), 797-815.

[24] Pandelis Dodos and Vassilis Kanellopoulos, On pairs of definable orthogonal families. Illinois J. Math. 52 (2008), no. 1, 181-201

[25] Alan Dow, PFA and $\omega_{1}^{*}$, Topology Appl. 28 (1988), no. 2, 127-140.

[26] Paul Erdős and András Hajnal, On chromatic number of graphs and set-systems, Acta Mathematica Academiae Scientiarum Hungaricae 17 (March, 1966), no. 1-2, pp. 61-99.

[27] Paul Erdős and Alfred Tarski, Annals of Mathematics, Second Series 44 (April, 1943), no. 2, pp. 315-329.

[28] Marián J. Fabian, Gteaux differentiability of convex functions and topology. A WileyInterscience Publication. John Wiley \& Sons, Inc., New York, 1997. xiv+180 pp. ISBN: $0-471-16822-\mathrm{X}$

[29] Ilijas Farah, OCA and reflection, Research Note of November 21, 1994.

[30] Ilijas Farah Seminar Notes on Problem 174, University of Toronto, December 1994.

[31] Ilijas Farah, Analytic Quotients: Theory of Liftings for Quotients over Analytic Ideals on the Integers Memoirs of the American Mathematical Society, Volume 148, Number 702 (2000), pp.1-177.

[32] Ilijas Farah and Jindrich Zapletal, Between Maharam's and von Neumann's problems. Math. Res. Lett. 11 (2004), no. 5-6, 673-684

[33] Ilijas Farah, Luzin gaps. Trans. Amer. Math. Soc. 356 (2004), no. 6, 2197-2239

[34] Ilijas Farah, Rigidity conjectures. Logic Colloquium 2000, 252-271, Lect. Notes Log., 19, Assoc. Symbol. Logic, Urbana, IL, 2005.

[35] Ilijas Farah, All automorphisms of the Calkin algebra are inner, March 2007.

[36] Qi Feng, Rado's conjecture and presaturation of the nonstationary ideal on $\omega_{1}$, Journal of Symbolic Logic 64 (March, 1999), no. 1, pp. 38-44.

[37] Qi Feng, Homogeneity for open partitions of pairs of reals, Transactions of the American Mathematical Society 339 (Oct., 1993), no. 2, pp. 659-684.

[38] Matthew Foreman, An $\aleph_{1}$-dense ideal on $\aleph_{2}$. Israel J. Math. 108 (1998), 253-290.

[39] Matthew Foreman, Menachem Maidor, Saharon Shelah, Martin's maximum, saturated ideals, and non-regular ultrafilters, Annals of Math., 127 (1988), 1-47.

[40] David H. Fremlin, Consequences of Martin's axiom, Cambridge University Press, 1984.

[41] David H. Fremlin, Perfect pre-images of $\omega_{i}$ and the PFA. Topology Appl. 29 (1988), no. 2, 151-166.

[42] David H. Fremlin, The partially ordered sets of measure theory and Tukey's ordering. Dedicated to the memory of Professor Gottfried Kthe. Note Mat. 11 (1991), 177-214.

[43] Fred Galvin, On Gruenhage's generalization of first countable spaces, II. Notices of Amer. Math. Soc., 24 (1977), A-257. 
[44] Moti Gitik, Nonsplitting subset of $\mathcal{P}_{\kappa}\left(\kappa^{+}\right)$. J. Symbolic Logic 50 (1985), no. 4, 881-894 (1986).

[45] James Hagler and William B. Johnson, On Banach spaces whose dual balls are not weak* sequentially compact. Israel J. Math. 28 (1977), no. 4, 325-330.

[46] Petr Hájek, Vicente Montesinos Santalucía, Jon Vanderwerff, Václav Zizler, Biorthogonal systems in Banach spaces. Springer, New York, 2008.

[47] Leo Harrington David Marker and Saharon Shelah, Borel orderings. Trans. Amer. Math. Soc. 310 (1988), no. 1, 293-302.

[48] Felix Hausdorff, Die Graduierung nach dem Endverlauf. Abh. König. Sächs. Ges. Wiss. Math.-Phys. Kl. 31 (1909), 296-334.

[49] Felix Hausdorff, Summen von $\aleph_{1}$ mengen, Fund. Math., 26 (1936), 241-255.

[50] James Hirschorn, Random trees under CH, Israel Journal of Mathematics 157 (Jan., 2007), no. 1 , pp. $123-153$.

[51] Alfred Horn and Alfred Tarski, Measures in Boolean Algebras, Transactions of the American Mathematical Society 64 (November, 1948), no. 3, pp. 467-497.

[52] John R. Isbell, Seven cofinal types, Journal of the London Mathematical Society, Second Series 4 (May, 1972), no. 4, pp. 651-654.

[53] Thomas Jech, Set theory. The third millennium edition, revised and expanded. Springer Monographs in Mathematics. Springer-Verlag, Berlin, 2003. xiv+769 pp.

[54] Ronald Jensen, The fine structure of the constructible hierarchy. With a section by Jack Silver. Ann. Math. Logic 4 (1972), 229-308; erratum, ibid. 4 (1972), 443.

[55] Ronald Jensen, Ernest Schimmerling, Ralf Schindler and John Steel, Stacking mice. J. Symbolic Logic 74 (2009), no. 1, 315-335.

[56] Mar Jiménez Sevilla, José P. Moreno, Renorming Banach spaces with the Mazur intersection property. J. Funct. Anal. 144 (1997), no. 2, 486-504.

[57] William B. Johnson and Haskell P. Rosenthal, On $\omega^{*}$-basic sequences and their applications to the study of Banach spaces. Studia Math. 43 (1972), 77-92

[58] Istvan Juhasz and Zoltan Szentmiklossy, Convergent free sequences in compact spaces. Proc. Amer. Math. Soc. 116 (1992), no. 4, 1153-1160.

[59] Winfried Just, Nowhere dense P-subsets of $\omega$. Proc. Amer. Math. Soc. 106 (1989), no. 4, $1145-1146$

[60] Winfried Just, Repercussions of a problem of Erdos and Ulam on density ideals. Canad. J. Math. 42 (1990), no. 5, 902-914.

[61] Winfried Just, A modification of Shelah's oracle-c.c. with applications. Trans. Amer. Math. Soc. 329 (1992), no. 1, 325-356.

[62] Winfried Just, A weak version of AT from OCA. Set theory of the continuum (Berkeley, CA, 1989), 281-291, Math. Sci. Res. Inst. Publ., 26, Springer, New York, 1992.

[63] Vladimir Kanovei and Michael Reeken, On Ulam's problem concerning the stability of approximate homomorphisms. Proc. Steklov Inst. Math. 2000, no. 4 (231), 238-270

[64] Alexander S. Kechris, Sławomir Solecki, and Stevo Todorcevic, Borel chromatic numbers, Advances in Mathematics 141 (1999), no. 1, pp. 1-44.

[65] Peter Komjáth, The chromatic number of some uncountable graphs. Sets, graphs and numbers (Budapest, 1991), 439-444, North-Holland, Amsterdam, 1992.

[66] Potr Koszmider, On a problem of Rolewich about Banach spaces that admit support sets, Preprint 2005.

[67] Kenneth Kunen, Set theory. An introduction to independence proofs. North-Holland Publishing Co., Amsterdam-New York, 1980. xvi+313 pp.

[68] Arkady G. Leiderman and Vitaly I. Malykhin, On preservation of the Lindelöf property in products of function spaces $C_{p}(X)$, Siberian Math. J. 29 (1988), 65-72.

[69] Jordi Lopez Abad and Stevo Todorcevic, Generic Banach spaces and generic simplexes, February 2010

[70] Donald A. Martin and John R. Steel, Projective determinacy. Proc. Nat. Acad. Sci. U.S.A. 85 (1988), no. 18, 6582-6586.

[71] Dorothy Maharam, An algebraic characterization of measure algebras, Annals of Mathematics, Second Series 48 (Jan., 1947), no. 1, pp. 154-167.

[72] R. Daniel Mauldin, The Scottish Book. Mathematics from the Scottish Caf. Birkhuser, Boston, Mass., 1981. xiii+268 pp. 
[73] Stanislaw Mazur, Uber schwache Konvergentz in en Raumen $L^{p}$, Studia Math., 4 (1933) 128-133.

[74] Benjamin Miller, Glimm-Effros dchotomies, 2009.

[75] Jan van Mill and George M. Reed Editors, Open problems in topology, North-Holland Publishing Co., Amsterdam, 1990. xiv+692 pp.

[76] Justin Tatch Moore, Open colorings, the continuum and the second uncountable cardinal, Proceedings of the American Mathematical Society 130 (2002), no. 9, pp. 2753-2759.

[77] Justin Tatch Moore, A solution to the L space problem. J. Amer. Math. Soc. 19 (2006), no. $3,717-736$

[78] Jorge Mujica, Separable quotients of Banach spaces. Rev. Mat. Univ. Complut. Madrid 10 (1997), no. 2, 299-330.

[79] Edward Odell and Haskell P. Rosenthal, A double-dual characterization of separable Banach spaces containing $l^{1}$. Israel J. Math. 20 (1975), no. 3-4, 375-384.

[80] Gert. K. Pedersen, Analysis now. Graduate Texts in Mathematics, vol. 118, Springer- Verlag, New York, 1989.

[81] N. Christopher Phillips and Nikolai Weaver, The Calkin algebra has outer automorphisms, Duke Mathematical Journal 139 (2007), no. 1, pp. 185-202.

[82] Sandra Quickert, CH and the Sacks property, Fundamenta Mathematicae 171 (2002), no. 1, pp. $93-100$.

[83] Richard Rado, Covering theorems for ordered sets, Proceedings of the London Mathematical Society (2) 50 (March, 1949), no. 7, pp. 509-535.

[84] Richard Rado, Theorems on intervals of ordered sets, Discrete Math., 35 (1981), 199-201.

[85] Stefan Rolewicz, On convex sets containing only points of support. Special issue dedicated to Wladyslaw Orlicz on the occasion of his seventy-fifth birthday. Comment. Math. Special Issue 1 (1978), 279-281

[86] Rosenthal, Haskell P. Point-wise compact subsets of the first Baire class. Amer. J. Math. 99 (1977), no. 2, 362-378

[87] Mary Ellen Rudin, Lectures on set theoretic topology. American Mathematical Society, Providence, R.I., 1975. iv +76 pp.

[88] Ernest Schimmerling and Martin Zeman, Characterization of $\square_{\kappa}$ in core models, Journal of Mathematical Logic, vol 4 (2004), pp 1-72.

[89] Ralf Shindler and John Steel The core model induction, book in preparation.

[90] Saharon Shelah, Proper forcing. Lecture Notes in Mathematics, 940. Springer-Verlag, BerlinNew York, 1982. xxix+496 pp.

[91] Saharon Shelah, Reflection implies the SCH. Fund. Math. 198 (2008), no. 2, 95-111

[92] Jack Silver, On the singular cardinals problem. Proceedings of the International Congress of Mathematicians (Vancouver, B. C., 1974), Vol. 1, pp. 265-268.

[93] Jack Silver, Counting the number of equivalence classes of Borel and coanalytic equivalence relations. Ann. Math. Logic 18 (1980), no. 1, 1-28

[94] Slawomir Solecki, Analytic ideals and their applications. Ann. Pure Appl. Logic 99 (1999), no. $1-3,51-72$.

[95] Slawomir Solecki and Stevo Todorcevic, Cofinal types of topological directed orders. Ann. Inst. Fourier (Grenoble) 54 (2004), no. 6, 1877-1911 (2005)

[96] Robert M. Solovay, On the cardinality of $\sum_{2}^{1}$ sets of reals, Foundations of Mathematics (Symposium Commemorating Kurt Gödel, Columbus, Ohio, 1966), Springer, New York, 1969, pp. 58-73.

[97] Robert M. Solovay, Strongly compact cardinals and the GCH, Proceedings of Symposia in Pure Mathematics, Vol. XXV, American Mathematical Society, Providence, Rhode Island, 1974, pp. 365-372.

[98] Robert M. Solovay, Real-Valued Measurable Cardinals, Axiomatic Set Theory (D. S. Scott, ed.), Procedings of the Symposium of Pure Mathamtics, vol. XIII, Part I, American Mathematics Society, Providence, 1971, pp. 397-428.

[99] John Steel, PFA implies $\mathrm{AD}^{L(\mathbb{R})}$, J. Symbolic Logic, 70(4) (2005), 1255-1296.

[100] Michel Talagrand, Maharam's problem, Ann. of Math. (2) 168 (2008), no. 3, 981-1009

[101] Stevo Todorcevic, Stationary sets, trees and continuums. Publ. Inst. Math. (Beograd) (N.S.) 29(43) (1981), 249-262.

[102] Stevo Todorcevic, Forcing positive partition relations. Trans. Amer. Math. Soc. 280 (1983), no. 2, 703-720. 
[103] Stevo Todorcevic, On a conjecture of R. Rado, Journal of the London Mathematical Society, Second Series 27 (1983), no. 1, pp. 1-8.

[104] Stevo Todorcevic, A note on the proper forcing axiom. Axiomatic set theory (Boulder, Colo., 1983), 209-218, Contemp. Math., 31, Amer. Math. Soc., Providence, RI, 1984

[105] Stevo Todorcevic, Reflection of stationary sets. Circulated note, 1984.

[106] Stevo Todorcevic, Directed sets and cofinal types. Trans. Amer. Math. Soc. 290 (1985), no. $2,711-723$.

[107] Stevo Todorcevic, Oscillations of real numbers, Logic colloquium '86 (Hull, 1986), Studies in Logic and the Foundations of Mathematics, vol. 124, North-Holland, Amsterdam, 1988, pp. $325-331$.

[108] Stevo Todorcevic, Partition Problems in Topology, Contemporary Mathematics, vol. 84, American Mathematical Society, Providence, Rhode Island, 1989.

[109] Stevo Todorcevic and Boban Velickovic, Martin's axiom and partitions. Compositio Math. 63 (1987), no. 3, 391-408.

[110] Stevo Todorcevic, PFA fails in the Sacks extension, Circulated Note of September 1987.

[111] Stevo Todorcevic, PFA implies $\omega_{2} \nrightarrow\left(\omega_{2}, \omega: 2\right)^{2}$, Circulated Note of January 1988.

[112] Stevo Todorcevic, Remarks on Martin's axiom and the continuum hypothesis. Canad. J. Math. 43 (1991), no. 4, 832-851.

[113] Stevo Todorcevic, Two examples of Borel partially ordered sets with the countable chain condition. Proc. Amer. Math. Soc. 112 (1991), no. 4, 1125-1128.

[114] Stevo Todorcevic, Conjectures of Rado and Chang and cardinal arithmetic, Finite and Infinite Combinatorics in Sets and Logic: Kluwer, Dordrecht, 1993, pp. 385-398.

[115] Stevo Todorcevic, Some applications of $S$ and L combinatorics. Ann. New York Acad. Sci., 705 (1993), 130-167.

[116] Stevo Todorcevic, Chang's Conjecture and Almost Disjoint Functions, Note of April 1995.

[117] Stevo Todorcevic, A classification of transitive relations on $\omega_{1}$. Proc. London Math. Soc. (3) 73 (1996), no. 3, 501-533.

[118] Stevo Todorcevic, Analytic gaps. Fund. Math. 150 (1996), no. 1, 55-66.

[119] Stevo Todorcevic, Comparing the continuum with the first two uncountable cardinals. Logic and scientific methods (Florence, 1995), 145-155, Synthese Lib., 259, Kluwer Acad. Publ., Dordrecht, 1997.

[120] Stevo Todorcevic, Embeddability of $K \times C$ into $X$. Bull. Cl. Sci. Math. Nat. Sci. Math. No. 22 (1997), 27-35.

[121] Stevo Todorcevic, Oscillations of sets of integers. Adv. in Appl. Math. 20 (1998), no. 2, $220-252$.

[122] Stevo Todorcevic Gaps in Analytic Quitients Fundamenta Mathematicae 156 (1998), no. 1, pp. 85-97.

[123] Stevo Todorcevic, Compact subsets of the first Baire class. J. Amer. Math. Soc. 12 (1999), no. $4,1179-1212$

[124] Stevo Todorcevic, A dichotomy for P-ideals of countable sets, Fundamenta Mathematicae 166 (2000), no. 3, pp. 251-267.

[125] Stevo Todorcevic, A problem of von Neumann and Maharam about algebras supporting continuous submeasures, Fundamenta Mathematicae 183 (2004), no. 2, pp. 169-183.

[126] Stevo Todorcevic, Biorthogonal systems and quotient spaces via Baire category methods. Math. Ann. 335 (2006), no. 3, 687-715.

[127] Stevo Todorcevic, Two possible cofinalities for infinite-dimensional Banach spaces, Research Note, June 2007.

[128] Stevo Todorcevic, Walks on ordinals and their characteristics. Progress in Mathematics, 263. Birkhäuser Verlag, Basel, 2007. vi+324 pp. ISBN: 978-3-7643-8528-6

[129] Stevo Todorcevic, Introduction to Ramsey spaces. Annals of Mathematics Studies, No. 174. Princeton University Press, 2010. 296 pp. ISBN13: 978-0-691-14541-9

[130] Stevo Todorcevic and Carlos Uzcátegui, Analytic k-spaces. Topology Appl. 146/147 (2005), $511-526$.

[131] John Wilder Tukey, Convergence and Uniformity in Topology, Annals of Mathematics Studies, no. 2, Princeton University Press, Princeton, N. J., 1940.

[132] Boban Velickovic, Definable automorphisms of $\mathcal{P}(\omega) /$ Fin. Proc. Amer. Math. Soc. 96 (1986), no. $1,130-135$

[133] Boban Velickovic, OCA and automorphisms of $\mathcal{P}(\omega) /$ Fin. Topology Appl. 49 (1993), 1-13. 
[134] Boban Velickovic, ccc forcing and splitting reals. Israel J. Math. 147 (2005), 209-220.

[135] Matteo Viale, A family of covering properties, Math. Research Letters, 15(2) (2008), pp. 221-238.

[136] W. Hugh Woodin, Supercompact cardinals, sets of reals, and weakly homogeneous trees, Proc. Nat. Acad. Sci., USA 85 (Sep. 15, 1988), no. 18, pp. 6587-6591.

[137] W. Hugh Woodin, The Axiom of Determinacy, Forcing Axioms, and the Nonstationary Ideal, De Gruyter, Berlin, New York, 1999.

Department of Mathematics, University of Toronto, Toronto, Ontario, Canada M5S 2E4

E-mail address: stevo@math.utoronto.ca 